Hepatic steatosis in familial combined hyperlipidemia 



\title{
Hepatic steatosis in familial combined hyperlipidemia
}

\author{
PROEFSCHRIFT
}

Ter verkrijging van de graad van doctor aan de Universiteit Maastricht, op gezag van de Rector Magnificius, Prof. Mr. G.P.M.F. Mols volgens het besluit van het College van Decanen, in het openbaar te verdedigen op vrijdag 7 maart 2008 om 14.00 uur

door

Martijn Carolus Gertrudis Johannes Brouwers geboren op 8 mei 1978 te Geldrop 


\section{Promotores:}

Prof. Dr. T.W.A. de Bruin

Prof. Dr. C.D.A. Stehouwer

\section{Co-promotores:}

Dr. M.M.J. van Greevenbroek

Dr. C.J.H. van der Kallen

\section{Beoordelingscommissie:}

Prof. Dr. M.H. Prins (voorzitter)

Dr. G.M. Dallinga-Thie (Universiteit van Amsterdam)

Dr. C.G. Schalkwijk

Prof. Dr. N.C. Schaper

Prof. Dr. A.F.H. Stalenhoef (Radboud Universiteit Nijmegen)

Financial support by the Netherlands Heart Foundation and the Dutch Diabetes Foundation for the publication of this thesis is gratefully acknowledged.

In addition, printing of this thesis was financially supported by AstraZeneca BV, Boehringer Ingelheim BV, Bristol-Myers Squibb BV, Genzyme Nederland BV, GlaxoSmithKline BV, J.E. Jurriaanse Stichting, Merck Sharp \& Dohme BV, Novartis Pharma BV, Novo Nordisk BV, Lilly BV, Pfizer BV, Sanofi-Aventis BV, Schering-Plough BV and Servier Nederland BV.

(C) Copyright Martijn C.G.J. Brouwers, Maastricht 2008

Universitaire Pers Maastricht

ISBN 978-90-5278-700-8 




\section{Table of contents}

1. Introduction 9

2. Original contributions 29

2.1 Genetic predisposition to develop fatty liver

2.1.1 Fatty liver is an integral feature of familial combined hyperlipidemia: 31 relation with fat distribution and plasma lipids

2.1.2 Five-year follow-up of waist circumference, insulin and ALT levels 44 in familial combined hyperlipidemia

2.1.3 Heritability and genetic loci of fatty liver in familial combined hyperlipidemia

2.2 Fatty liver based subgroups

2.2.1 Fatty liver based identification of two distinct hypertriglyceridemic 77 subgroups in familial combined hyperlipidemia

2.2.2 Inflammation, endothelial function and fibrinolysis in relation to fatty liver and hypertriglyceridemia in familial combined hyperlipidemia

2.3 Fatty liver in relation to the multiple-type hyperlipidemia

2.3.1 Longitudinal differences in familial combined hyperlipidemia quantitative trait loci

2.3.2 Parabolic relation between plasma triglycerides and LDLcholesterol: long-term follow-up in the general population, type 2 diabetes mellitus and familial combined hyperlipidemia

3. General discussion

4.1 Summary 146

$\begin{array}{lll}4.2 & \text { Samenvatting } & 148\end{array}$

4.3 Dankwoord 150

4.4 Curriculum vitae $\quad 155$

4.5 Scientific output 156

Appendix A: list of abbreviations

Appendix B: linkage results for fatty liver 163 
Introduction

Historical and epidemiological background of FCHL Pathogenesis of FCHL: a working hypothesis The multiple-type hyperlipidemia and diagnostic problems Aims and outlines of this thesis 


\subsection{Historical and epidemiological background of familial combined hyperlipidemia}

In 1967, Donald Fredrickson and colleagues were the first to provide a phenotypic classification of plasma lipid abnormalities. They showed that five distinct groups of lipid abnormalities can be recognized by the simple use of paper electrophoresis [1-3] (Figure 1). Six years later, Joseph Goldstein and colleagues applied Fredrickson's criteria to survivors of myocardial infarction and reported the presence of three common familial lipid disorders [4]: 1) familial hypercholesterolemia (Fredrickson's class IIa), 2) familial hypertriglyceridemia (Fredrickson's class IV) and 3) familial combined hyperlipidemia, a newly discovered hyperlipidemia, allegedly inherited as an autosomal dominant disease and characterized by the presence of different Fredrickson's subtypes within one family, i.e. hypercholesterolemia (IIa), hypertriglyceridemia (IV) or the combination of both (IIb). In that same year, two other groups independently reported very similar results $[5,6]$. It was estimated that this new lipid disorder, FCHL in short, is highly prevalent in the general population (1:100) and among patients with premature myocardial infarction (1:10) [4]. Subsequent cross-sectional and longitudinal studies revealed that first-degree relatives of a FCHL index patient are also at increased risk (2-5 times) to develop myocardial infarction $[7,8]$. This increased risk combined with the high prevalence of FCHL in the general population underlines the clinical relevance of this disease.

In the past decades it has been demonstrated that many FCHL patients share features of the metabolic syndrome [9] (Table 1), such as insulin resistance [10-13], visceral obesity [14], hypertension [15], the presence of small-dense LDL particles [16-19] and low HDL concentrations. Many of these features have independently been associated with an increased cardiovascular risk [20-24] and are therefore all expected to contribute to the increased cardiovascular risk in FCHL.

Table 2 summarizes the diagnostic criteria of FCHL as described in the early seventies that have been adopted (with small modifications) by our laboratory.

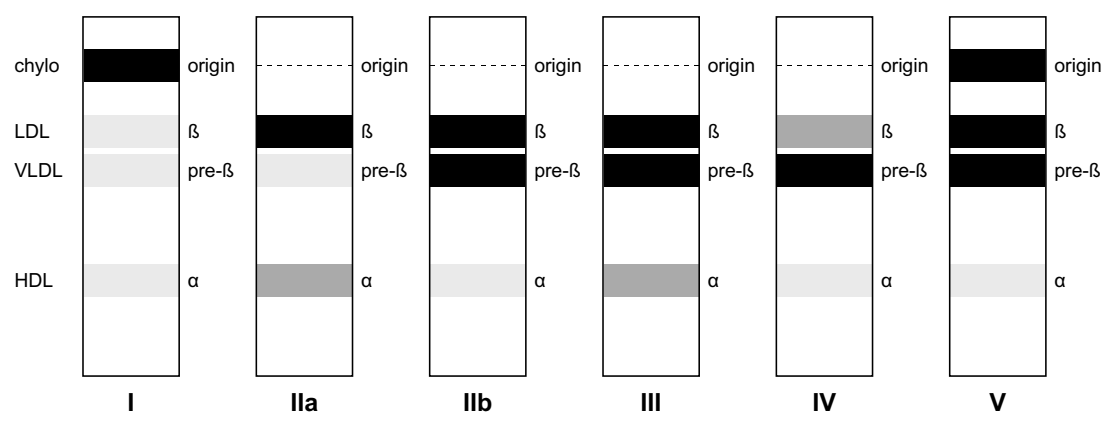

Figure 1.

Fredrickson's classification of lipid disorders by paper electrophoresis (type IIb hyperlipidemia was later added) 
Table 1.

Metabolic Syndrome as defined by the World Health Organization (WHO) and Adult Treatment Panel III (ATPIII)

WHO:

Insulin resistance, identified by one of the following: type 2 diabetes mellitus, impaired fasting glucose, impaired glucose tolerance or for those with normal fasting glucose levels, glucose uptake below the lowest quartile for background population under investigation under hyperinsulinemic, euglycemic conditions.

Plus any two of the following:

- Use of antihypertensive medication and/or high blood pressure (>140 $\mathrm{mmHg}$ systolic or $>90 \mathrm{mmHg}$ diastolic)

- $\quad$ Plasma triglycerides $>1.7 \mathrm{mmol} / \mathrm{L}$

- HDL-cholesterol $<0.9 \mathrm{mmol} / \mathrm{L}$ in men, $<1.0 \mathrm{mmol} / \mathrm{L}$ in women

- $\quad$ BMI $>30 \mathrm{~kg} / \mathrm{m}^{2}$ and/or waist-to-hip ratio $>0.9$ in men, $>0.85$ in women

- Urinary albumin excretion rate $>20 \mu \mathrm{g} / \mathrm{min}$ or albumin:creatinine ratio $>30 \mathrm{mg} / \mathrm{g}$

ATPIII:

At least three of the following criteria:

- Abdominal obesity, i.e. waist circumference $>102 \mathrm{~cm}$ in men, $>88 \mathrm{~cm}$ in women

- Plasma triglycerides $>1.7 \mathrm{mmol} / \mathrm{L}$

- HDL-cholesterol $<1.03 \mathrm{mmol} / \mathrm{L}$ in men, $<1.3 \mathrm{mmol} / \mathrm{L}$ in women

- Blood pressure $>130 \mathrm{mmHg}$ systolic and/or $>85 \mathrm{mmHg}$ diastolic

- $\quad$ Fasting glucose $>6.1 \mathrm{mmol} / \mathrm{L}$

Table 2.

Diagnostic criteria of Familial Combined Hyperlipidemia

Inclusion criteria:

- $\quad$ Presence of premature myocardial infarction, i.e. before age 60 years, in one family

- Presence of at least two different hyperlipidemic phenotypes, i.e. hypercholesterolemia (class IIa), hypertriglyceridemia (class IV) or both (class IIb), in one family

Exclusion criteria:

- Other primary causes of hyperlipidemia, such as familial hypercholesterolemia and familial dysbetalipoproteinemia, diagnosed by the presence of tendon xanthomas and E2/E2 genotype, respectively

- Secondary causes of hyperlipidemia in the proband, such as obesity (body mass index $>$ $30 \mathrm{~kg} / \mathrm{m} 2$ ), diabetes mellitus, and dysfunction of thyroid, kidney and liver 


\subsection{Pathogenesis of FCHL: a working hypothesis}

Shortly after the first description of FCHL, it was shown that, in contrast to what was previously assumed, FCHL is not inherited as an autosomal dominant disorder, but is in fact a complex disease $[18,19,25,26]$. This implicates that the phenotype of FCHL is caused by the interaction of susceptibility genes in combination with environmental factors.

It is the current understanding that the hyperlipidemia in FCHL is the consequence of an increased production of VLDL-particles as well as a decreased clearance of remnants. At present, many genetic studies have been conducted to unravel the clearance defects in FCHL, whereas mainly metabolic studies have underlined the concept of overproduction as a cause of hyperlipidemia in FCHL. These studies will be reviewed in the following paragraphs.

\subsubsection{Overproduction of lipid particles (Figure 2)}

Numerous studies have demonstrated that FCHL patients overproduce apo B containing particles resulting in increased plasma lipid levels [27-31]. This overproduction is most likely driven by an increased hepatic supply of fatty acids in combination with an insulin resistant state. The organs that play a key role in energy homeostasis, i.e. adipose tissue, muscle and liver, are anticipated to play a causal role:

\subsubsection{Evidence for dysfunctional adipose tissue in FCHL}

In time of energy surplus, i.e. during the postprandial state, glucose (converted to glycerol) and fatty acids are stored as triglycerides in adipose tissue. The opposite takes place during the fasting state when there is a demand for energy: fatty acids are liberated by hormone sensitive lipase (HSL) and enter the circulation as a substrate for other organs. Insulin is one the most important hormones that regulates the uptake, storage and release of fatty acids, among others by suppressing HSL.

The evidence for dysfunctional adipose tissue in FCHL is abundant. Fasting and postprandial plasma free fatty acids (FFA) are increased in FCHL and insensitive to the suppressive effects of insulin [10-12, 32-34]. Some researchers have attributed these in vivo observations to an insulinresistant HSL, resulting in an excess release of FFAs into the circulation [34, 35]. However, in vitro studies have reported contrasting results [36-38], and gene-expression, linkage and association studies have demonstrated that at the most a modifying or interacting role can be assigned to HSL in the pathogenesis of FCHL [12, 38-41]. Others have explained the in vivo FFA elevation by a defective adipocyte FFA uptake, either as a primary cause or secondary to an impaired triglyceride storage $[12,42,43]$. To date, the exact defect has remained unknown. Besides a dysfunctional, insulin-resistant adipose tissue, it is likely that the total amount and distribution of fat tissue play a role in the pathogenesis of FCHL as well. FCHL patients are generally more obese, in particular in the abdominal region [14]. Since omental fat tissue drains directly into to the portal vein towards the liver, all FFAs that are released from this depot will enter the liver and can be used as a substrate for the VLDL-production. 


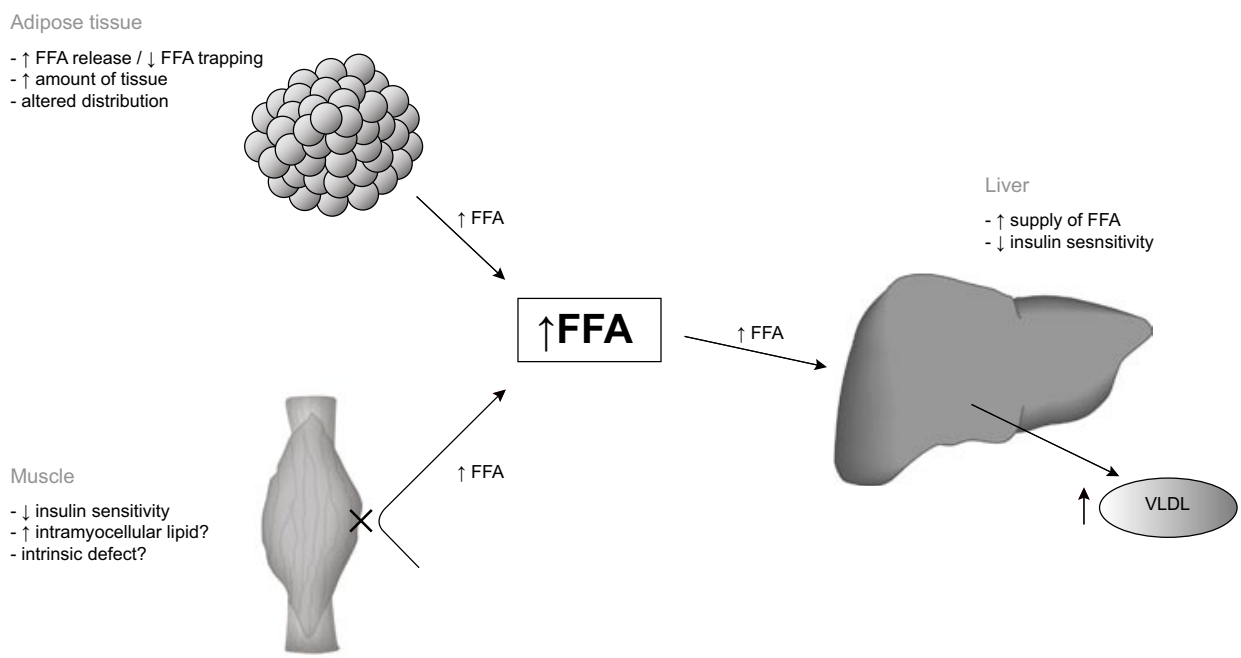

Figure 2.

Schematic overview of hypothesized pathogenesis of VLDL-overproduction in FCHL

However, even after adjustment for (abdominal) obesity, FCHL patients are more susceptible to develop hyperlipidemia $[44,45]$, implying that the amount and distribution of fat may contribute to the FCHL phenotype, but are not the principal cause.

\subsubsection{The insulin-resistant muscle}

Several hyperinsulinemic euglycemic clamp studies have demonstrated that the glucose infusion rate to maintain steady glucose concentrations is lower in FCHL patients, indicating that FCHL patients are more insulin resistant than controls [10-13, 33]. Aitman et al. concluded that the resistance to suppress glucose levels under hyperinsulinemc conditions is confined to the muscle, because hepatic glucose output was normally suppressed in FCHL patients during the clamp procedure [10]. Since studies in other metabolically related diseases have shown that muscle insulin resistance is related with intramuscular lipid accumulation [46-48], it is tempting to speculate that this could also be the case in FCHL. Whether an increased intramuscular lipid accumulation is due to a primary muscle defect or to an increased FFA supply from dysfunctional adipose tissue remains to be elucidated as well. Of interest, by using indirect calorimetry techniques, Karjalainen et al. have shown that glucose oxidation is decreased and lipid oxidation increased not only in FCHL patients, but also in their first-degree normolipidemic relatives, suggesting that the metabolic disturbances in muscle may precede the hyperlipidemia in FCHL [33].

\subsubsection{Increased FFA supply and hepatic insulin resistance in relation to VLDL-overproduction}


Increased plasma levels of FFA, the resultant of either an impaired storage or an increased release by dysfunctional adipose tissue, and hypothetically of an impaired uptake by a triglyceride-filled muscle, is anticipated to lead to an increased supply to the liver. Of interest, varying plasma FFA levels affect the hepatic VLDL-production in both normal subjects and patients with type 2 diabetes mellitus $[49,50]$. In addition, hepatic insulin resistance appears to play a role in this process as well, since insulin suppresses the production of VLDL-particles, in particular the triglyceride-rich VLDL1-particles [51-53]. This mechanism is disturbed in patients with type 2 diabetes mellitus: the VLDL1-production does not decrease after administration of insulin, which is indicative of an insulin-resistant liver [54]. It is therefore expected that at least two biological determinants are a prerequisite for VLDL-overproduction in FCHL: an increased hepatic FFA supply and hepatic insulin resistance.

\subsubsection{Decreased clearance of lipid particles (Figure 3)}

Oral fat load tests have demonstrated that the clearance of chylomicrons and VLDL-particles is impaired in FCHL patients [55-58]. Since these two triglyceride-rich particles compete with each other for the same clearance mechanisms, it is expected that the increased amounts of liverderived VLDL-particles result in an increased competition and thus a delayed clearance [55-58]. Several genetic studies have shown, that besides this relative impairment, absolute clearance defects can account for the fasting and postprandial hyperlipidemia as well.

Triglycerides are predominantly secreted as buoyant, triglyceride-rich VLDL1 (Svedberg flotation $\left[\mathrm{S}_{\mathrm{f}}\right]$ rate 60-400) or as smaller, triglyceride-poorer VLDL2 $\left(\mathrm{S}_{\mathrm{f}} 20-60\right)$ particles. VLDL2-particles are abundant at low plasma triglycerides concentration $(<1.5 \mathrm{mmol} / \mathrm{L})$, whereas the relative and absolute number of VLDL1-particles rapidly rises as soon as plasma triglycerides increase above $1.5 \mathrm{mmol} / \mathrm{L}$ [59]. This has also been observed in FCHL [17]. In normal subjects it is estimated that almost half of the secreted VLDL-1 particles are directly removed from plasma by different receptors, i.e. the VLDL-receptor, the LDL-related protein (LRP) and heparan sulfate proteoglycans (HSPG), with apolipoprotein E serving as a ligand. The remainder of the VLDL1-particles is delipidated mainly by lipoprotein lipase (LPL). Apolipoprotein CII and $\mathrm{E}$ are co-factors in this process, whereas apolipoprotein CIII antagonizes the LPL-mediated catabolism of VLDL1 [59]. Of interest, genetic variants in the apo-AI-CIII-AIV-AV cluster on chromosome 11q23-24, have been linked to and associated with triglyceride levels in FCHL [7, 60-66]. In addition, genetic studies have attributed a modifying role to LPL in the expression of FCHL [35, 40, 62, 63, 66-73].

VLDL2-particles arise either after delipidation of VLDL1 or by direct hepatic production. They are catabolized by either LPL or hepatic lipase (HL) into IDL-particles, which are further converted into LDL-particles by HL. VLDL2-, IDL- and LDL-particles can be cleared from plasma by the LDL-receptor, mediated by apolipoprotein B [59]. Genetic variants in the HL gene have been shown to influence plasma lipid levels in FCHL [67-69]. However, HL is not expected to be a major FCHL gene [41]. LDL-receptor defects have not been observed in FCHL, since they are characteristic of familial hypercholesterolemia, an exclusion criterion of 


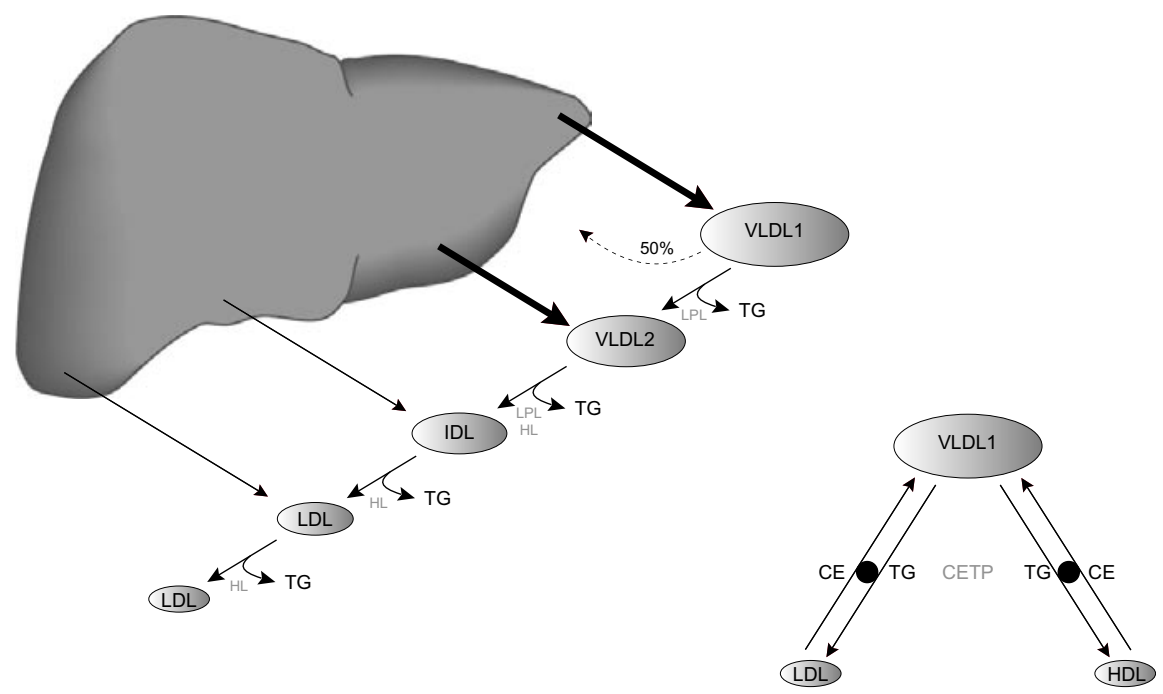

Figure 3.

Catabolic cascade of triglyceride-rich particles secreted by the liver (left) and CETP mediated formation of smalldense LDL-particles and reduced HDL-cholesterol levels (right)

FCHL (Table 2).

In addition to the increased quantity of LDL-particles, the quality of the lipid content carried in LDL particles is altered in the majority of FCHL patients [16-19]. Their LDL-particles are smaller and more cholesterol- and triglycerides-depleted, the so-called small-dense (sd) or pattern B LDL, opposite to buoyant or pattern A LDL that are predominant in normal subjects. SdLDL originates when VLDL1-particles become abundant in plasma, i.e. at plasma triglycerides above $1.5 \mathrm{mmol} / \mathrm{L}-\mathrm{a}$ frequent finding in FCHL subjects. VLDL1-particles exchange their triglycerides for cholesteryl esters (CE) with LDL-particles, which is mediated by cholesteryl ester transfer protein (CETP) (Figure 3). The triglycerides in the cholesterol-depleted LDL-particles are subsequently hydrolyzed by HL resulting in the characteristic sdLDL-particles [59, 70, 71]. A very similar process takes places between VLDL1- and HDL-particles resulting in reduced HDL-cholesterol levels [59, 72]. The triad: elevated VLDL1, sdLDL and low HDL-cholesterol was coined 'the atherogenic lipoprotein phenotype' (ALP) [73] and it has been documented that it is associated with a three times increased risk for cardiovascular disease [20]. Several whole genome linkage analyses have been conducted for HDL-cholesterol and LDL-particle size in FCHL [18, 25, 74-76]. Although linkage has often been documented in the 16q region (which harbors CETP), CETP does not appear to be a major causative gene for reduced HDLcholesterol levels and the presence of sdLDL in FCHL [18, 25, 74, 75]. 


\subsection{The multiple-type hyperlipidemia and diagnostic problems}

Diagnosing FCHL is a demanding procedure, since it requires a family screening for at least two different lipid phenotypes, the so-called multiple-type hyperlipidemia. Many researchers have therefore argued for a more simple, unitary diagnosis of FCHL [77-79]. The need for new diagnostic criteria was further emphasized by the observation of Brunzell et al. that the multipletype hyperlipidemia could also be present within one individual, depending on genetic and environmental factors [80]. This assumption was later confirmed in two long-term longitudinal studies: 28 to $34 \%$ of all FCHL family members changed lipid phenotype over time [81, 82]. Of more concern, some individuals were found to be affected at one time point, but unaffected during the other measurement $[81,82]$. This misclassification can have detrimental effects on (genetic) studies that use the affected state to elucidate the complex pathogenesis of FCHL, but also on the choices for treatment of an individual FCHL patient. Efforts have therefore been made to unravel the causes of the multiple-type hyperlipidemia within one FCHL individual and to redefine FCHL with variables that are less variable over time. Veerkamp et al. reported that the switch in triglyceride phenotype was associated with changes in BMI and insulin levels and that apolipoprotein (apo) B levels are less variable over time [82, 83]. In addition, apo B levels have been shown to be a consistent hallmark of all lipid phenotypes of FCHL $[16,82]$ and to be a risk factor for cardiovascular complications [84]. Different research groups have therefore proposed to include apo B levels as a new diagnostic criterion of FCHL [78, 82, 85].

\subsection{Aims and outlines of this thesis}

\subsubsection{Recent findings relevant for this thesis}

In the early eighties, Ludwig and colleagues described histological abnormalities of the liver that were indistinguishable from alcoholic hepatitis in patients who denied alcohol abuse [86]. This new liver entity, nonalcoholic steatohepatitis (NASH), appeared to be associated with obesity and obesity-related disorders, such as diabetes mellitus and cholelithiasis [86]. During the last decade, NASH has gained much more attention of both hepatologists and endocrinologists due to its increased risk of progression to the potential lethal end-stage cirrhosis $[86,87]$ and its supposed active involvement in the pathogenesis of the metabolic syndrome [88]. Brunt et al. incorporated NASH into a broader spectrum, i.e. nonalcoholic fatty liver disease (NAFLD), which histologically comprises simple steatosis, steatohepatitis, fibrosis and end-stage cirrhosis [89]. In addition to type 2 diabetes mellitus [86], congenital lipodistrophy [90] and highly active anti-retroviral therapy (HAART) associated lipodystrophy [91], FCHL was very recently added to the list of metabolic syndrome-related disorders that are characterized by an increased prevalence of NAFLD [92]. Our laboratory demonstrated that outpatient FCHL patients, in particular patients with the hypertriglyceridemic phenotype, exhibit radiological signs of fatty liver, the first stage of NAFLD [92]. This observation is consistent with our working hypothesis as described previously: the well-documented overproduction of VLDL-particles in FCHL is the consequence of an increased hepatic FFA supply and hepatic insulin resistance [32, 54]. As 
outlined in Figure 4, it is very plausible that an increased hepatic supply of FFA will result in an increased amount of fat in the liver. Hepatic fat accumulation could subsequently lead to an insulin resistant liver, analogous to what has been observed in muscle [46-48]. An insulin-resistant VLDL-pathway could mechanistically account for the overproduction of VLDL-particles.

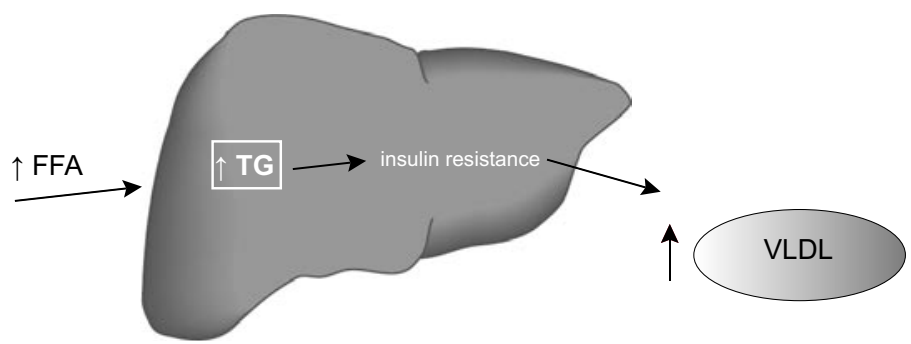

Figure 4.

Hepatic fat (TG) accumulation could be the missing link between the well-documented factors contributing to VLDLoverproduction in FCHL, i.e. increased FFA-flux and hepatic insulin resistance

\subsubsection{Aims and outlines of this thesis}

In general terms, the key theme of this thesis is:

'Extension and refinement of molecular and clinical mechanisms in FCHL by assessing the role of fatty liver'

This theme has been dissected into three lines of research with specific questions (aims)

1. Genetic predisposition to develop fatty liver in FCHL (paragraph 2.1); as mentioned earlier, the elevated plasma lipid levels are the consequence of both genetic and environmental factors. Our laboratory has nicely illustrated this complex pathogenesis by showing that for every increment in waist-to-hip ratio (WHR) category, a factor that is markedly influenced by unhealthy lifestyle habits (i.e. the environmental factor), the risk for developing hyperlipidemia increases in FCHL (Figure 5). However, FCHL subjects still have a higher risk of developing hyperlipidemia in comparison with spouses with the same WHR, which is suggestive of a genetic susceptibility component (Figure 5) [44]. It would be of interest to observe whether similar outcomes would be obtained when replacing the hyperlipidemia by the fatty liver trait. In other words: are FCHL patients, with similar increments in obesity, more prone to develop fatty liver in comparison with controls?

Paragraph 2.1.1 and 2.1.2 will deal with this question, cross-sectionally and longitudinally, respectively. Finally, paragraph 2.1 .3 will provide a definite answer to whether fatty liver is really a heritable aspect of FCHL. 


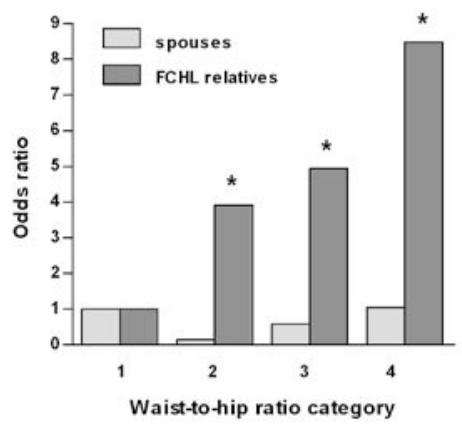

Figure 5.

Odds ratio for hypertriglyceridemia in FCHL relatives and spouses stratified by waist-to-hip ratio. Odds ratio in waistto-hip category 1 is set to $1 .^{*} \mathrm{p}<0.05$ in comparison to waist-to-hip category 1 [44].

2. Refinement of FCHL phenotype by inclusion of the fatty liver trait (paragraph 2.2); according to our theory, we anticipate that fatty liver will be particularly linked to the VLDL-overproduction pathway and to a much lesser extent to the impaired clearance pathway. It is therefore plausible that distinct subgroups can be identified within FCHL, in particular within the hypertriglyceridemic phenotype, by using the fatty liver trait. This option will be explored in paragraph 2.2.1. The applicability of the fatty liver trait for the identification of subgroups with different cardiovascular risk profiles will be investigated in paragraph 2.2.2.

3. Fatty liver in relation to the multiple-type hyperlipidemia (paragraph 2.3); the intraindividual variations in plasma triglycerides and total cholesterol, which are responsible for the multiple-type hyperlipidemia in FCHL, can potentially influence the outcomes of genetic studies that use these lipid variables as a trait. The impact of these individual variations on the outcomes of quantitative trait locus (QTL) analyses will be studied in paragraph 2.3.1.

Since elevated plasma triglycerides are related to fatty liver in FCHL [92], it would be of interest to observe a similar relationship between the longitudinal variations in fatty liver and plasma triglycerides. Such a correlation could account for the switch in triglyceride phenotype in FCHL. This issue will be addressed in paragraph 2.1.2, in addition to the increased susceptibility to develop fatty liver.

The liver could also be more distantly linked to the switch in cholesterol phenotype, assuming that plasma triglycerides and cholesterol are related with each other. This is not unconceivable since triglycerides and cholesteryl esters are both transported by apo B containing particles. The longitudinal relation between triglycerides and LDLcholesterol will be described in paragraph 2.3.2. 


\subsubsection{Surrogates of fatty liver}

In this thesis, ultrasound of the liver and ALT levels have been used as validated surrogate markers of fatty liver. The 'gold standard' to diagnose fatty liver and to distinguish its presence from hepatitis or fibrosis is a liver biopsy [89]. However, liver biopsies are not acceptable without any clinical indication and are thus not a suitable research tool to detect hepatic fat accumulation in our relatively healthy FCHL population. Alternative methods, such as computed tomography (CT), magnetic resonance imaging (MRI) and magnetic resonance spectroscopy (MRS) are valid measures to detect the amount of hepatic fat (in particular the latter method) [93], but they share the disadvantage that they are too expensive to be used in large research cohorts. From the practical point of view, these radiological techniques are often only available to research at night or in the weekend as the equipment is in use for regular patient care. It was a logical choice to use ultrasound as a versatile, practical, low cost tool for human research.

The application of ultrasound in the detection of fatty liver received much attention in the late seventies and eighties within the framework of diagnosing alcoholic fatty liver disease [94-103]. Nowadays, ultrasound has again gained much attention, this time in the scope of diagnosing nonalcoholic fatty liver disease [104]. The ultrasonographical features of fatty liver are all the consequence of the same principle: the presence of lipid droplets in the hepatocytes causes more irregular transitions for ultrasound waves, which results in increased reflections (= increased intensities, 'bright liver') and thus decreased penetrance (posterior beam attenuation), and scattering of the ultrasound signal (= decreased resolution, impaired visualization of hepatic vessels) [95, 99-101]. These features have been incorporated into a semi-quantitative diagnosis of fatty liver (Figure 6) [104]. Validation studies have shown that the sensitivity to detect mild stages of fatty liver with ultrasound is moderate, whereas high sensitivity rates $(>95 \%)$ have been found for moderate to severe stages of fatty liver [94-96, 98, 100, 102-104]. The reported specificity is high as well, although it may be difficult to distinguish fatty liver from fibrosis [94$98,100,102,103]$. One can conclude that ultrasound is a relatively cheap, well-validated and non-invasive modality to detect fatty liver in medical research. The major disadvantage however is that it is at best a semi-quantitative surrogate of fatty liver, in contrast to a continuous measure, which is preferable in for example longitudinal studies.

There is some evidence that the liver enzymes aspartate aminotransferase (AST) and alanine aminotransferase (ALT) can also be used as surrogates of NAFLD. Clark et al. reported that these easily obtainable, quantitative markers were associated with many features of the metabolic syndrome after exclusion of alternative chronic diseases, e.g. alcoholic liver disease, hepatitis B/ $\mathrm{C}$ infections and haemochromatosis [105]. ALT and AST may reflect the presence of NAFLD, a frequent finding in subjects with metabolic syndrome [105]. 


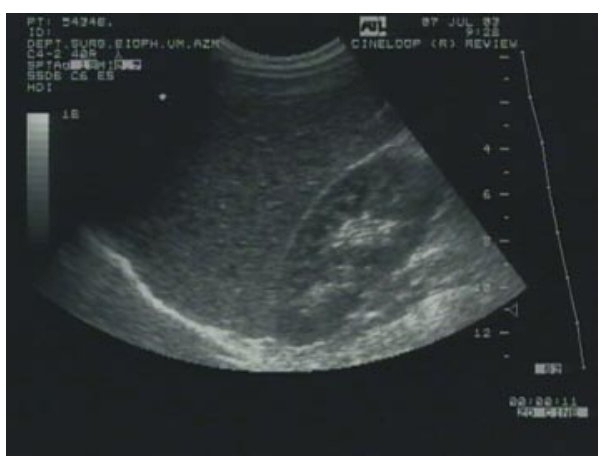

Normal

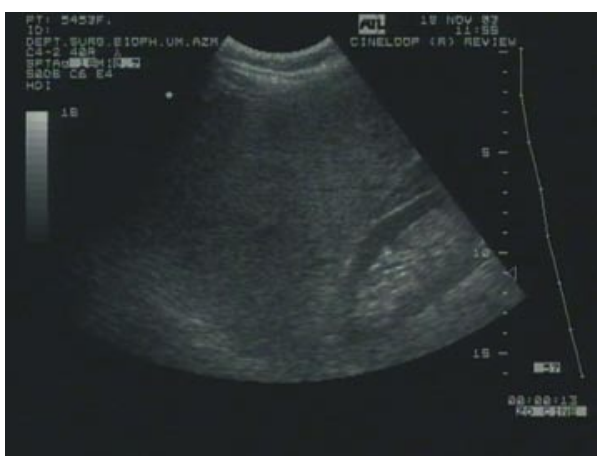

Moderate

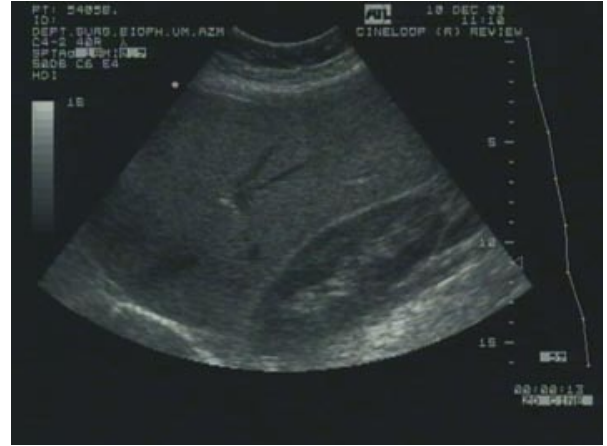

Mild

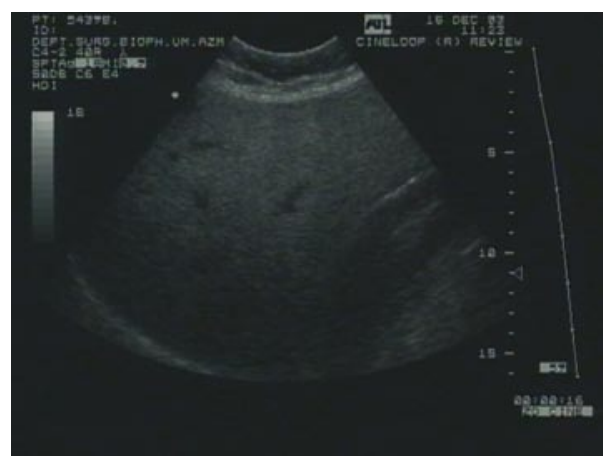

Severe

\section{Figure 6.}

Stages of fatty liver, ranging from normal to severe fatty liver, as measured with ultrasound [104].

Normal liver: normal hepatic echotexture and normal beam attenuation;

Mild steatosis: slight increase in echogenicity of liver parenchyma in comparison with right kidney, with minimal or no decrease of visualization of hepatic vessels and diaphragm;

Moderate steatosis: diffuse increase in echogenicity of the liver with slightly impaired visualization of intrahepatic vessels and diaphragm;

Severe steatosis: marked increase of liver echogenicity, poor visualization of intrahepatic vessels and increased posterior beam attenuation represented by poor visualization of the diaphragm. 


\subsection{References}

[1] Fredrickson DS, Levy RI, Lees RS (1967) Fat transport in lipoproteins--an integrated approach to mechanisms and disorders.PG - 148-56 contd. N Engl J Med 276

[2] Fredrickson DS, Levy RI, Lees RS (1967) Fat transport in lipoproteins--an integrated approach to mechanisms and disorders.PG - 215-25 contd. N Engl J Med 276

[3] Fredrickson DS, Levy RI, Lees RS (1967) Fat transport in lipoproteins--an integrated approach to mechanisms and disorders.PG - 273-81 concl. N Engl J Med 276

[4] Goldstein JL, Schrott HG, Hazzard WR, Bierman EL, Motulsky AG (1973) Hyperlipidemia in coronary heart disease. II. Genetic analysis of lipid levels in 176 families and delineation of a new inherited disorder, combined hyperlipidemia. J Clin Invest 52: 1544-1568

[5] Nikkila EA, Aro A (1973) Family study of serum lipids and lipoproteins in coronary heart disease. Lancet 1: 954-959

[6] Rose HG, Kranz P, Weinstock M, Juliano J, Haft JI (1973) Inheritance of combined hyperlipoproteinemia: evidence for a new lipoprotein phenotype. Am J Med 54: 148-160

[7] Voors-Pette C, de Bruin TW (2001) Excess coronary heart disease in Familial Combined Hyperlipidemia, in relation to genetic factors and central obesity. Atherosclerosis 157:481489

[8] Austin MA, McKnight B, Edwards KL, et al. (2000) Cardiovascular disease mortality in familial forms of hypertriglyceridemia: A 20-year prospective study. Circulation 101:27772782

[9] Grundy SM, Brewer HB, Jr., Cleeman JI, Smith SC, Jr., Lenfant C (2004) Definition of metabolic syndrome: Report of the National Heart, Lung, and Blood Institute American Heart Association conference on scientific issues related to definition. Circulation 109: 433-438

[10] Aitman TJ, Godsland IF, Farren B, Crook D, Wong HJ, Scott J (1997) Defects of insulin action on fatty acid and carbohydrate metabolism in familial combined hyperlipidemia Arterioscler Thromb Vasc Biol 17: 748-754

[11] Pihlajamaki J, Karjalainen L, Karhapaa P, Vauhkonen I, Laakso M (2000) Impaired free fatty acid suppression during hyperinsulinemia is a characteristic finding in familial combined hyperlipidemia, but insulin resistance is observed only in hypertriglyceridemic patients. Arterioscler Thromb Vasc Biol 20: 164-170

[12] van der Kallen CJ, Voors-Pette C, Bouwman FG, et al. (2002) Evidence of insulin resistant lipid metabolism in adipose tissue in familial combined hyperlipidemia, but not type 2 diabetes mellitus. Atherosclerosis 164: 337-346

[13] Bredie SJ, Tack CJ, Smits P, Stalenhoef AF (1997) Nonobese patients with familial combined hyperlipidemia are insulin resistant compared with their nonaffected relatives Arterioscler Thromb Vasc Biol 17: 1465-1471

[14] Purnell JQ, Kahn SE, Schwartz RS, Brunzell JD (2001) Relationship of insulin sensitivity and ApoB levels to intra-abdominal fat in subjects with familial combined hyperlipidemia Arterioscler Thromb Vasc Biol 21: 567-572 
[15] Keulen ET, Voors-Pette C, de Bruin TW (2001) Familial dyslipidemic hypertension syndrome: familial combined hyperlipidemia, and the role of abdominal fat mass. Am J Hypertens 14: 357-363

[16] Ayyobi AF, McGladdery SH, McNeely MJ, Austin MA, Motulsky AG, Brunzell JD (2003) Small, Dense LDL and Elevated Apolipoprotein B Are the Common Characteristics for the Three Major Lipid Phenotypes of Familial Combined Hyperlipidemia. Arterioscler Thromb Vasc Biol 23: 1289-1294

[17] Georgieva AM, van Greevenbroek MM, Krauss RM, et al. (2004) Subclasses of low density lipoprotein and very low-density lipoprotein in familial combined hyperlipidemia relationship to multiple lipoprotein phenotype. Arterioscler Thromb Vasc Biol 24: 744-749

[18] Jarvik GP, Brunzell JD, Austin MA, Krauss RM, Motulsky AG, Wijsman E (1994) Genetic predictors of FCHL in four large pedigrees. Influence of ApoB level major locus predicted genotype and LDL subclass phenotype. Arterioscler Thromb 14: 1687-1694

[19] Austin MA, Brunzell JD, Fitch WL, Krauss RM (1990) Inheritance of low density lipoprotein subclass patterns in familial combined hyperlipidemia. Arteriosclerosis 10: $520-530$

[20] Austin MA, Breslow JL, Hennekens CH, Buring JE, Willett WC, Krauss RM (1988) Low density lipoprotein subclass patterns and risk of myocardial infarction. Jama 260: 1917 1921

[21] Kannel WB, Cupples LA, Ramaswami R, Stokes J, 3rd, Kreger BE, Higgins M (1991) Regional obesity and risk of cardiovascular disease; the Framingham Study. J Clin Epidemiol 44: 183-190

[22] Kannel WB, Dawber TR, Friedman GD, Glennon WE, McNamara PM (1964) Risk Factors in Coronary Heart Disease. An Evaluation of Several Serum Lipids as Predictors of Coronary Heart Disease; the Framingham Study. Ann Intern Med 61: 888-899

[23] Kannel WB, Dawber TR, Thomas HE, Jr., McNamara PM (1965) Comparison of Serum Lipids in the Prediction of Coronary Heart Disease. Framingham Study Indicates That Cholesterol Level and Blood Pressure Are Major Factors in Coronary Heart Disease; Effect of Obesity and Cigarette Smoking Also Noted. R I Med J 48: 243-250

[24] Austin MA, Hokanson JE, Edwards KL (1998) Hypertriglyceridemia as a cardiovascular risk factor. Am J Cardiol 81: 7B-12B

[25] Austin MA, Wijsman E, Guo SW, Krauss RM, Brunzell JD, Deeb S (1991) Lack of evidence for linkage between low-density lipoprotein subclass phenotypes and the apolipoprotein B locus in familial combined hyperlipidemia. Genet Epidemiol 8: 287-297

[26] Shoulders CC, Jones EL, Naoumova RP (2004) Genetics of familial combined hyperlipidemia and risk of coronary heart disease. Hum Mol Genet 13 Spec No 1: R149-160

[27] Venkatesan S, Cullen P, Pacy P, Halliday D, Scott J (1993) Stable isotopes show a direct relation between VLDL apoB overproduction and serum triglyceride levels and indicate a metabolically and biochemically coherent basis for familial combined hyperlipidemia Arterioscler Thromb 13: 1110-1118 
[28] Chait A, Albers JJ, Brunzell JD (1980) Very low density lipoprotein overproduction in genetic forms of hypertriglyceridaemia. Eur J Clin Invest 10: 17-22

[29] Janus ED, Nicoll AM, Turner PR, Magill P, Lewis B (1980) Kinetic bases of the primary hyperlipidaemias: studies of apolipoprotein B turnover in genetically defined subjects. Eur J Clin Invest 10: 161-172

[30] Kissebah AH, Alfarsi S, Adams PW (1981) Integrated regulation of very low density lipoprotein triglyceride and apolipoprotein-B kinetics in man: normolipemic subjects, familial hypertriglyceridemia and familial combined hyperlipidemia. Metabolism 30: 856-868

[31] Cortner JA, Coates PM, Bennett MJ, Cryer DR, Le NA (1991) Familial combined hyperlipidaemia: use of stable isotopes to demonstrate overproduction of very lowdensity lipoprotein apolipoprotein B by the liver. J Inherit Metab Dis 14: 915-922

[32] Castro Cabezas M, de Bruin TW, de Valk HW, Shoulders CC, Jansen H, Willem Erkelens D (1993) Impaired fatty acid metabolism in familial combined hyperlipidemia. A mechanism associating hepatic apolipoprotein B overproduction and insulin resistance. J Clin Invest 92: 160-168

[33] Karjalainen L, Pihlajamaki J, Karhapaa P, Laakso M (1998) Impaired insulin-stimulated glucose oxidation and free fatty acid suppression in patients with familial combined hyperlipidemia: a precursor defect for dyslipidemia? Arterioscler Thromb Vasc Biol 18: 1548-1553

[34] Meijssen S, Derksen RJ, Bilecen S, Erkelens DW, Cabezas MC (2002) In vivo modulation of plasma free fatty acids in patients with familial combined hyperlipidemia using lipid lowering medication. J Clin Endocrinol Metab 87: 1576-1580

[35] Meijssen S, Cabezas MC, Twickler TB, Jansen H, Erkelens DW (2000) In vivo evidence of defective postprandial and postabsorptive free fatty acid metabolism in familial combined hyperlipidemia. J Lipid Res 41: 1096-1102

[36] Reynisdottir S, Angelin B, Langin D, et al. (1997) Adipose tissue lipoprotein lipase and hormone-sensitive lipase. Contrasting findings in familial combined hyperlipidemia and insulin resistance syndrome. Arterioscler Thromb Vasc Biol 17: 2287-2292

[37] Reynisdottir S, Eriksson M, Angelin B, Arner P (1995) Impaired activation of adipocyte lipolysis in familial combined hyperlipidemia. J Clin Invest 95: 2161-2169

[38] Ylitalo K, Large V, Pajukanta P, et al. (2000) Reduced hormone-sensitive lipase activity is not a major metabolic defect in Finnish FCHL families. Atherosclerosis 153: 373-381

[39] Ylitalo K, Nuotio I, Viikari J, Auwerx J, Vidal H, Taskinen MR (2002) C3, hormone sensitive lipase, and peroxisome proliferator-activated receptor gamma expression in adipose tissue of familial combined hyperlipidemia patients. Metabolism 51: 664-670

[40] Pihlajamaki J, Valve R, Karjalainen L, Karhapaa P, Vauhkonen I, Laakso M (2001) The hormone sensitive lipase gene in familial combined hyperlipidemia and insulin resistance. Eur J Clin Invest 31: 302-308

[41] Pajukanta P, Porkka KV, Antikainen M, et al. (1997) No evidence of linkage between familial combined hyperlipidemia and genes encoding lipolytic enzymes in Finnish families Arterioscler Thromb Vasc Biol 17: 841-850 
[42] Sniderman AD, Cianflone K, Arner P, Summers LK, Frayn KN (1998) The adipocyte, fatty acid trapping, and atherogenesis. Arterioscler Thromb Vasc Biol 18: 147-151

[43] Halkes CJ, Van Dijk H, Verseyden C, et al. (2003) Gender Differences in Postprandial Ketone Bodies in Normolipidemic Subjects and in Untreated Patients With Familial Combined Hyperlipidemia. Arterioscler Thromb Vasc Biol

[44] van der Kallen CJ, Voors-Pette C, de Bruin TW (2004) Abdominal obesity and expression of familial combined hyperlipidemia. Obes Res 12: 2054-2061

[45] Ascaso JF, Sales J, Merchante A, et al. (1997) Influence of obesity on plasma lipoproteins, glycaemia and insulinaemia in patients with familial combined hyperlipidaemia. Int J Obes Relat Metab Disord 21: 360-366

[46] Krssak M, Falk Petersen K, Dresner A, et al. (1999) Intramyocellular lipid concentrations are correlated with insulin sensitivity in humans: a 1H NMR spectroscopy study. Diabetologia 42: 113-116

[47] Dagenais GR, Tancredi RG, Zierler KL (1976) Free fatty acid oxidation by forearm muscle at rest, and evidence for an intramuscular lipid pool in the human forearm. J Clin Invest 58: 421-431

[48] Pan DA, Lillioja S, Kriketos AD, et al. (1997) Skeletal muscle triglyceride levels are inversely related to insulin action. Diabetes 46: 983-988

[49] Lewis GF, Uffelman KD, Szeto LW, Weller B, Steiner G (1995) Interaction between free fatty acids and insulin in the acute control of very low density lipoprotein production in humans. J Clin Invest 95: 158-166

[50] Malmstrom R, Packard CJ, Caslake M, et al. (1998) Effects of insulin and acipimox on VLDL1 and VLDL2 apolipoprotein B production in normal subjects. Diabetes 47: 779-787

[51] Malmstrom R, Packard CJ, Watson TD, et al. (1997) Metabolic basis of hypotriglyceridemic effects of insulin in normal men. Arterioscler Thromb Vasc Biol 17: 1454-1464

[52] Gill JM, Brown JC, Bedford D, et al. (2004) Hepatic production of VLDL1 but not VLDL2 is related to insulin resistance in normoglycaemic middle-aged subjects. Atherosclerosis 176: 49-56

[53] Brown AM, Gibbons GF (2001) Insulin inhibits the maturation phase of VLDL assembly via a phosphoinositide 3-kinase-mediated event. Arterioscler Thromb Vasc Biol 21: 1656-1661

[54] Malmstrom R, Packard CJ, Caslake M, et al. (1997) Defective regulation of triglyceride metabolism by insulin in the liver in NIDDM. Diabetologia 40: 454-462

[55] Castro Cabezas M, Erkelens DW, Kock LA, De Bruin TW (1994) Postprandial apolipoprotein B100 and B48 metabolism in familial combined hyperlipidaemia before and after reduction of fasting plasma triglycerides. Eur J Clin Invest 24: 669-678

[56] Cabezas MC, de Bruin TW, Jansen H, Kock LA, Kortlandt W, Erkelens DW (1993) Impaired chylomicron remnant clearance in familial combined hyperlipidemia. Arterioscler Thromb 13: 804-814

[57] Reitsma JB, Castro Cabezas M, de Bruin TW, Erkelens DW (1994) Relationship between 
improved postprandial lipemia and low-density lipoprotein metabolism during treatment with tetrahydrolipstatin, a pancreatic lipase inhibitor. Metabolism 43: 293-298

[58] Verseyden C, Meijssen S, Castro Cabezas M (2002) Postprandial changes of apoB-100 and apoB-48 in TG rich lipoproteins in familial combined hyperlipidemia. J Lipid Res 43: 274-280

[59] Packard CJ, Shepherd J (1997) Lipoprotein heterogeneity and apolipoprotein metabolism Arterioscler Thromb Vasc Biol 17: 3542-3556

[60] Wojciechowski AP, Farrall M, Cullen P, et al. (1991) Familial combined hyperlipidaemia linked to the apolipoprotein AI-CII-AIV gene cluster on chromosome 11q23-q24. Nature 349: 161-164

[61] Xu CF, Talmud P, Schuster H, Houlston R, Miller G, Humphries S (1994) Association between genetic variation at the APO AI-CIII-AIV gene cluster and familial combined hyperlipidaemia. Clin Genet 46: 385-397

[62] Ribalta J, La Ville AE, Vallve JC, Humphries S, Turner PR, Masana L (1997) A variation in the apolipoprotein C-III gene is associated with an increased number of circulating VLDL and IDL particles in familial combined hyperlipidemia. J Lipid Res 38: 1061-1069

[63] Aouizerat BE, Allayee H, Cantor RM, et al. (1999) Linkage of a candidate gene locus to familial combined hyperlipidemia: lecithin:cholesterol acyltransferase on 16q. Arterioscler Thromb Vasc Biol 19: 2730-2736

[64] Coon H, Myers RH, Borecki IB, et al. (2000) Replication of linkage of familial combined hyperlipidemia to chromosome 1q with additional heterogeneous effect of apolipoprotein A-I/C-III/A-IV locus. The NHLBI Family Heart Study. Arterioscler Thromb Vasc Biol 20: $2275-2280$

[65] Eichenbaum-Voline S, Olivier M, Jones EL, et al. (2003) Linkage and Association Between Distinct Variants of the APOA1/C3/A4/A5 Gene Cluster and Familial Combined Hyperlipidemia. Arterioscler Thromb Vasc Biol

[66] Mar R, Pajukanta P, Allayee H, et al. (2004) Association of the APOLIPOPROTEIN A1/C3/A4/A5 gene cluster with triglyceride levels and LDL particle size in familial combined hyperlipidemia. Circ Res 94: 993-999

[67] Hoffer MJ, Snieder H, Bredie SJ, et al. (2000) The V73M mutation in the hepatic lipase gene is associated with elevated cholesterol levels in four Dutch pedigrees with familial combined hyperlipidemia. Atherosclerosis 151: 443-450

[68] Pihlajamaki J, Karjalainen L, Karhapaa P, et al. (2000) G-250A substitution in promoter of hepatic lipase gene is associated with dyslipidemia and insulin resistance in healthy control subjects and in members of families with familial combined hyperlipidemia. Arterioscler Thromb Vasc Biol 20: 1789-1795

[69] Allayee H, Dominguez KM, Aouizerat BE, et al. (2000) Contribution of the hepatic lipase gene to the atherogenic lipoprotein phenotype in familial combined hyperlipidemia. J Lipid Res 41: 245-252

[70] Vakkilainen J, Jauhiainen M, Ylitalo K, et al. (2002) LDL particle size in familial combined hyperlipidemia: effects of serum lipids, lipoprotein-modifying enzymes, and lipid transfer proteins. J Lipid Res 43: 598-603 
[71] Demacker PN, Veerkamp MJ, Bredie SJ, Marcovina SM, de Graaf J, Stalenhoef AF (2000) Comparison of the measurement of lipids and lipoproteins versus assay of apolipoprotein B for estimation of coronary heart disease risk: a study in familial combined hyperlipidemia. Atherosclerosis 153: 483-490

[72] Soro A, Jauhiainen M, Ehnholm C, Taskinen MR (2003) Determinants of low HDL levels in familial combined hyperlipidemia. J Lipid Res 44: 1536-1544

[73] Austin MA, King MC, Vranizan KM, Krauss RM (1990) Atherogenic lipoprotein phenotype. A proposed genetic marker for coronary heart disease risk. Circulation 82: 495-506

[74] Allayee H, Aouizerat BE, Cantor RM, et al. (1998) Families with familial combined hyperlipidemia and families enriched for coronary artery disease share genetic determinants for the atherogenic lipoprotein phenotype. Am J Hum Genet 63: 577-585

[75] Badzioch MD, Igo RP, Jr., Gagnon F, et al. (2004) Low-density lipoprotein particle size loci in familial combined hyperlipidemia: evidence for multiple loci from a genome scan. Arterioscler Thromb Vasc Biol 24: 1942-1950

[76] Soro A, Pajukanta P, Lilja HE, et al. (2002) Genome scans provide evidence for low-HDL C loci on chromosomes 8q23, 16q24.1-24.2, and 20q13.11 in Finnish families. Am J Hum Genet 70: 1333-1340

[77] Sniderman AD, Ribalta J, Castro Cabezas M (2001) How should FCHL be defined and how should we think about its metabolic bases? Nutr Metab Cardiovasc Dis 11: 259-273

[78] Sniderman AD, Castro Cabezas M, Ribalta J, et al. (2002) A proposal to redefine familial combined hyperlipidaemia -- third workshop on FCHL held in Barcelona from 3 to 5 May 2001, during the scientific sessions of the European Society for Clinical Investigation. Eur J Clin Invest 32: 71-73

[79] Porkka KV, Nuotio I, Pajukanta P, et al. (1997) Phenotype expression in familial combined hyperlipidemia. Atherosclerosis 133: 245-253

[80] Brunzell JD, Albers JJ, Chait A, Grundy SM, Groszek E, McDonald GB (1983) Plasma lipoproteins in familial combined hyperlipidemia and monogenic familial hypertriglyceridemia. J Lipid Res 24: 147-155

[81] McNeely MJ, Edwards KL, Marcovina SM, Brunzell JD, Motulsky AG, Austin MA (2001) Lipoprotein and apolipoprotein abnormalities in familial combined hyperlipidemia: a 20 year prospective study. Atherosclerosis 159: 471-481

[82] Veerkamp MJ, de Graaf J, Bredie SJ, Hendriks JC, Demacker PN, Stalenhoef AF (2002) Diagnosis of familial combined hyperlipidemia based on lipid phenotype expression in 32 families: results of a 5-year follow-up study. Arterioscler Thromb Vasc Biol 22: 274-282

[83] Veerkamp MJ, de Graaf J, Stalenhoef AF (2005) Role of insulin resistance in familial combined hyperlipidemia. Arterioscler Thromb Vasc Biol 25: 1026-1031

[84] Lamarche B, Despres JP, Moorjani S, Cantin B, Dagenais GR, Lupien PJ (1995) Prevalence of dyslipidemic phenotypes in ischemic heart disease (prospective results from the Quebec Cardiovascular Study). Am J Cardiol 75: 1189-1195

[85] Veerkamp MJ, de Graaf J, Hendriks JC, Demacker PN, Stalenhoef AF (2004) Nomogram to diagnose familial combined hyperlipidemia on the basis of results of a 5-year follow- 
up study. Circulation 109: 2980-2985

[86] Ludwig J, Viggiano TR, McGill DB, Oh BJ (1980) Nonalcoholic steatohepatitis: Mayo Clinic experiences with a hitherto unnamed disease. Mayo Clin Proc 55: 434-438

[87] Lee RG (1989) Nonalcoholic steatohepatitis: a study of 49 patients. Hum Pathol 20: 594598

[88] Kelley DE, McKolanis TM, Hegazi RA, Kuller LH, Kalhan SC (2003) Fatty liver in type 2 diabetes mellitus: relation to regional adiposity, fatty acids, and insulin resistance. Am J Physiol Endocrinol Metab 285: E906-916

[89] Brunt EM, Janney CG, Di Bisceglie AM, Neuschwander-Tetri BA, Bacon BR (1999) Nonalcoholic steatohepatitis: a proposal for grading and staging the histological lesions. Am J Gastroenterol 94: 2467-2474

[90] Powell EE, Searle J, Mortimer R (1989) Steatohepatitis associated with limb lipodystrophy. Gastroenterology 97: 1022-1024

[91] Miller KD, Cameron M, Wood LV, Dalakas MC, Kovacs JA (2000) Lactic acidosis and hepatic steatosis associated with use of stavudine: report of four cases. Ann Intern Med 133: 192-196

[92] de Bruin TW, Georgieva AM, Brouwers MC, Heitink MV, van der Kallen CJ, van Greevenbroek MM (2004) Radiological evidence of nonalcoholic fatty liver disease in familial combined hyperlipidemia. Am J Med 116: 847-849

[93] Siegelman ES, Rosen MA (2001) Imaging of hepatic steatosis. Semin Liver Dis 21: 71-80

[94] Foster KJ, Dewbury KC, Griffith AH, Wright R (1980) The accuracy of ultrasound in the detection of fatty infiltration of the liver. Br J Radiol 53: 440-442

[95] Gosink BB, Lemon SK, Scheible W, Leopold GR (1979) Accuracy of ultrasonography in diagnosis of hepatocellular disease. AJR Am J Roentgenol 133: 19-23

[96] Taylor KJ, Gorelick FS, Rosenfield AT, Riely CA (1981) Ultrasonography of alcoholic liver disease with histological correlation. Radiology 141: 157-161

[97] Debongnie JC, Pauls C, Fievez M, Wibin E (1981) Prospective evaluation of the diagnostic accuracy of liver ultrasonography. Gut 22: 130-135

[98] Scatarige JC, Scott WW, Donovan PJ, Siegelman SS, Sanders RC (1984) Fatty infiltration of the liver: ultrasonographic and computed tomographic correlation. J Ultrasound Med 3:9-14

[99] Sanford NL, Walsh P, Matis C, Baddeley H, Powell LW (1985) Is ultrasonography useful in the assessment of diffuse parenchymal liver disease? Gastroenterology 89: 186-191

[100] Saverymuttu SH, Joseph AE, Maxwell JD (1986) Ultrasound scanning in the detection of hepatic fibrosis and steatosis. Br Med J (Clin Res Ed) 292: 13-15

[101] Taylor KJ, Riely CA, Hammers L, et al. (1986) Quantitative US attenuation in normal liver and in patients with diffuse liver disease: importance of fat. Radiology 160: 65-71

[102] Celle G, Savarino V, Picciotto A, Magnolia MR, Scalabrini P, Dodero M (1988) Is hepatic ultrasonography a valid alternative tool to liver biopsy? Report on 507 cases studied with both techniques. Dig Dis Sci 33: 467-471

[103] Joseph AE, Saverymuttu SH, al-Sam S, Cook MG, Maxwell JD (1991) Comparison of liver histology with ultrasonography in assessing diffuse parenchymal liver disease. Clin 
Radiol 43: 26-31

[104] Saadeh S, Younossi ZM, Remer EM, et al. (2002) The utility of radiological imaging in nonalcoholic fatty liver disease. Gastroenterology 123: 745-750

[105] Clark JM, Brancati FL, Diehl AM (2003) The prevalence and etiology of elevated aminotransferase levels in the United States. Am J Gastroenterol 98: 960-967 
2.

Original contributions

Genetic predisposition to develop fatty liver

Fatty liver based subgroups Fatty liver in relation to the multiple-type hyperlipidemia 
Genetic predisposition to develop fatty liver

Fatty liver is an integral feature of familial combined hyperlipidemia: relation with fat distribution and plasma lipids

Brouwers MC, Bilderbeek-Beckers MA Georgieva AM, van der Kallen CJ van Greevenbroek MM de Bruin TW

Clin Sci (Lond). 2007; 112(2):123-130 


\section{Abstract}

Overproduction of VLDL-particles is an important cause of familial combined hyperlipidemia (FCHL). It was recently shown that the VLDL-production is driven by the amount of hepatic fat.

The present study was conducted to determine the prevalence of fatty liver in relation to the different fat compartments and lipid parameters in FCHL.

Sixty-eight FCHL patients, 110 normolipidemic relatives and 66 spouses underwent ultrasound of the abdominal region to estimate the amount of subcutaneous, visceral and hepatic fat. Skinfold callipers were used to measure subcutaneous fat of the biceps, triceps, subscapular and supra-iliacal regions.

Fatty liver was observed in $18 \%$ of the spouses, $25 \%$ of the normolipidemic relatives and $49 \%$ of the FCHL patients. After adjustment for age, gender and body mass index, the prevalence of fatty liver was significantly higher in FCHL patients versus spouses ( $p=0.03$, OR: 3.1), and also in the normolipidemic relatives versus spouses $(\mathrm{p}=0.02$, OR: 4.0$)$, whereas no differences were observed between FCHL patients and normolipidemic relatives ( $p=0.58$, OR: 0.8$)$. In the normolipidemic relatives and FCHL patients combined, both visceral fat mass and subcutaneous abdominal fat were independent predictors of fatty liver $(p<0.001$ for both fat compartments; FCHL status corrected). Of interest, fatty liver stages were correlated with both VLDL-apo B and VLDL-triglycerides in a representative subset of patients and relatives $\left(n=69 ; r^{2}=0.12\right.$, $\mathrm{p}=0.006$ and $\mathrm{r}^{2}=0.18, \mathrm{p}=0.001$, respectively).

These data show that fatty liver is a central aspect of FCHL, i.e. patients and normolipidemic relatives. Both visceral and subcutaneous adiposity contribute to its three to four fold higher risk in FCHL. 


\section{Introduction}

More than 30 years ago, familial combined hyperlipidemia (FCHL) was delineated as a genetic hyperlipidemia, characterized by the presence of different hyperlipidemic phenotypes, i.e. isolated hypercholesterolemia, isolated hypertriglyceridemia or the combination of both, within one family [1]. It was estimated that FCHL is highly prevalent (1:00) in the general population and even more abundant (1:10) among survivors of a premature myocardial infarction [1]. More recently it was shown that the presence of small-dense LDL-particles and elevated apolipoprotein B levels are consistent features of FCHL $[2,3]$. Furthermore, most FCHL subjects exhibit many features of the metabolic syndrome, such as insulin resistance [4] and abdominal obesity $[5,6]$. Several studies have demonstrated that, although the expression of FCHL very much depends on the amount of (abdominal) fat [7], FCHL patients are still more hyperlipidemic when corrected or matched for the degree of obesity [7, 8]. These observations underline the complex pathogenesis of FCHL: both environmental and genetic factors are responsible for the FCHL phenotype.

The characteristic hyperlipidemia of FCHL is the consequence of both a hepatic overproduction of very low-density lipoproteins (VLDL) and an impaired clearance of remnant particles [9-11]. Adiels et al. very recently reported that the overproduction of VLDL-particles is driven by the amount of fat that is accumulated in the liver in normal subjects and patients with type 2 diabetes mellitus [12]. Of interest, we recently demonstrated that the prevalence of an increased amount of hepatic fat, i.e. fatty liver, was drastically increased in a small group of outpatient FCHL patients in comparison with community controls [13]. Given these recent findings, it is anticipated that the fatty liver trait will play a very important role in the expression of the FCHL phenotype.

The aim of the present study was to extent and refine current knowledge of fatty liver in FCHL. For this we estimated the prevalence of fatty liver not only in a larger sample of FCHL patients, but also in their normolipidemic relatives, derived from our 21 well-defined FCHL families. These prevalence numbers were subsequently compared with their spouses, who are exposed to a similar environment. Furthermore, analogous to the reported contribution of abdominal obesity to the expression of FCHL [7], we investigated whether abdominal obesity, more specifically visceral and abdominal subcutaneous obesity, contribute to the expression of fatty liver in FCHL. Finally, we correlated plasma insulin, apolipoprotein B and triglycerides levels, three hallmarks of FCHL [2, 3, 14], with the different stages of fatty liver.

\section{Materials and methods}

\section{Study population}

FCHL family members (i.e. FCHL patients and their normolipidemic relatives) and spouses of ages ranging between 20 and 70 years were invited to participate in this study. Diagnosis of FCHL was established as described previously [15]. The FCHL affected state was defined as plasma levels of total cholesterol $>6.5 \mathrm{mmol} / \mathrm{L}$ and/or triglycerides $>2.3 \mathrm{mmol} / \mathrm{L}$ (classical criteria [1]). More recently it was proposed to redefine FCHL according to plasma triglycerides 
levels $>1.5 \mathrm{mmol} / \mathrm{L}$ and apolipoprotein (apo) B $>1.2 \mathrm{~g} / \mathrm{L}$ [14]. Some of our analyses were repeated with these newly proposed criteria to exclude the possibility that our observations depend on one particular definition of the affected and non-affected state.

Subjects visited the clinical research centre after an overnight fast and abstinence from alcohol for the last three days. Lipid lowering medication was stopped two weeks prior to the visit. Furthermore, subjects had a stable weight, did not take any medication associated with the development of fatty liver [16], and consumed no more than $20 \mathrm{~g}$ of alcohol daily. One subject was found to be seropositive for hepatitis $C$ and was therefore excluded from the present study.

The study protocol was approved by the Human Investigations Review Committee at Maastricht University/Academic Hospital Maastricht. All subjects gave written informed consent.

\section{Anthropometric measurements}

Subjects were weighed in their underwear, height was determined by a stadiometer and body mass index (BMI) was calculated by weight $(\mathrm{kg})$ divided by height $(\mathrm{m})$ squared. Hip circumference was determined in standing position at the level of the trochanter major. Skinfold thickness was measured with plastic calliper at left biceps, triceps, subscapular and suprailiacal site. Waist circumference and sagittal diameter were measured in supine position at the level midway between the iliacal crest and lowest rib at the end of a normal expiration. Sagittal diameter was measured with a sliding beam calliper. Waist to hip ratio (WHR) was calculated as the ratio between waist and hip circumference.

\section{Ultrasound}

All ultrasound measurements were performed with an ATL 9 HDI ultrasound (US) system (Bothel, USA). Both visceral adipose tissue (VAT-US) and subcutaneous adipose tissue (SATUS) were determined at the same level as waist circumference and sagittal diameter. SAT-US was measured at the midline as the distance between skin and linea alba, using a L5-10 transducer. VAT-US was determined as the distance between the anterior of the vertebrate body and the peritoneum, exactly as described by Stolk et al [17]. All measurements were done by the same researcher with minimal pressure exerted on the probe and at the end of a normal expiration. The intra-observer variability, as determined in 30 subjects, was $1.7 \%$.

Fatty liver was detected by ultrasound as well, using a C7-4 and C4-2 transducer. Standardized images of the liver and right kidney were recorded on videotape and examined by an independent radiologist unaware of the subject's clinical characteristics. The severity of fatty liver was graded into mild, moderate and severe fatty liver by using the criteria of posterior beam attenuation, increased echogenicity ('bright liver') and decreased visualization of intrahepatic vessels, as described previously $[18,19]$. The intra-observer agreement expressed as kappa, determined in 30 random scans, was substantial for both the presence of fatty liver (present/absent) $(x=$ $0.74)$ and the different fatty liver stages (normal, mild, moderate, severe) $(x=0.68)$. This is in agreement with earlier studies [18]. 


\section{Laboratory measurements}

Plasma total cholesterol, triglyceride, apo B and insulin concentrations were measured as previously described [20]. VLDL isolation was done as described by Redgrave et al. [21]. Apolipoprotein B concentration in this fraction was quantified by gel electrophoresis according to the method of Karpe et al [22].

\section{Statistical analyses}

Differences in continous and binary variables between spouses, normolipidemic relatives and FCHL patients, included as dummy variables, were tested with mulivariate linear and logistic regression, respectively.

Univariate correlations of anthropometric variables, plasma insulin, triglycerides and apo B levels with the different stages of fatty liver were calculated with ordinal regression in all FCHL family members. Several multivariate ordinal regression models were constructed to study the independent effects of different anthropometric variables on the prediction of fatty liver. The FCHL patients and normolipidemic relatives were included in these models as dummy variable. The overall goodness of fit of the model was expressed as Nagelkerke's pseudo r-squared that serves the same function as r-squared in linear regression models.

SPSS 13.0 for Windows was used for all statitistical analyses (SPSS Inc., Chicago, Illinois).

\section{Results}

\section{General and anthropometric characteristics of FCHL patients, normolipidemic relatives and spouses}

In total, 178 FCHL family members (68 FCHL patients and 110 normolipidemic relatives), and 66 spouses from 21 families participated in this study. Table 1 shows their general and anthropometric characteristics. Since the normolipidemic (NL) relatives were signficantly younger than the spouses and FCHL patients, all analyses were adjusted for age and sex. By definition, FCHL patients had higher total cholesterol and serum triglycerides values compared with NL relatives and spouses. Furthermore, FCHL patients were more obese. This was explained, at least in part, by an increased visceral fat depot reflected by increased waist circumference, WHR, sagittal diameter, and more directly assessed by increased VAT-US. Furthermore, the subcutaneous fat compartment also seemed to be increased, as expressed by increased SATUS, biceps and subscapular skinfolds. The latter two were only siginifcantly different from the spouses. However, other markers of subcutaneous adipose tissue, i.e. hip circumference, triceps skinfold and suprailiacal skinfold, were not statistically different between the three groups. Of interest, both WHR and VAT-US were significantly higher in NL relatives in comparison with spouses (Table 1).

\section{Prevalence of fatty liver in FCHL patients, NL relatives and their spouses}

The prevelance of fatty liver measured with ultrasound was $49 \%$ in FCHL patients, which was significantly higher in comparison with the $18 \%$ in spouses $(p=0.003$, Odds ratio [OR]: 2.9; $95 \% \mathrm{CI}: 1.5-5.5$ ), but not with the $25 \%$ as observed in the NL relatives ( $\mathrm{p}=0.18$, OR: 1.6 ; $95 \% \mathrm{CI}: 0.8-3.3$ ), after correction for age and sex (Table 1). Of interest, when these analyses 
Table 1.

General and anthropometric characteristics of FCHL patiens, NL relatives and spouses

\begin{tabular}{|c|c|c|c|}
\hline & Spouses & NL relatives & FCHL patients \\
\hline Male / Female & $34 / 32$ & $50 / 60$ & $31 / 37$ \\
\hline Age, years & $48.0(43.0-56.3)$ & $37.5(28.8-52.0)^{*}$ & $54.0(46.5-60.0)$ \\
\hline Total Cholesterol, mmol/L & $5.3 \pm 1.0$ & $5.0 \pm 0.8$ & $7.2 \pm 2.0+\ddagger$ \\
\hline Triglycerides, mmol/L & $1.1(0.8-1.7)$ & $1.1(0.9-1.5)$ & $2.6(1.6-3.5) \dagger \neq$ \\
\hline Apolipoprotein B, g/L & $1.0 \pm 0.2$ & $1.0 \pm 0.3$ & $1.3 \pm 0.3+\ddagger$ \\
\hline Insulin, $\mathrm{U} / \mathrm{L}$ & $4.6(2.0-8.7)$ & $5.4(2.0-8.9)$ & $9.5(6.9-13.7) \dagger \neq$ \\
\hline Lipid medication, $\%$ & 6 & 10 & $50+\ddagger$ \\
\hline $\mathrm{BMI}, \mathrm{kg} / \mathrm{m} 2$ & $25.4 \pm 4.2$ & $25.2 \pm 4.3$ & $28.2 \pm 3.9+\ddagger$ \\
\hline Waist circumference $(\mathrm{cm})$ & $89.2 \pm 12.4$ & $88.2 \pm 12.1$ & $97.5 \pm 9.9+\neq$ \\
\hline Hip circumference $(\mathrm{cm})$ & $100.6 \pm 8.2$ & $100.4 \pm 8.0$ & $104.1 \pm 8.0$ \\
\hline WHR & $0.88 \pm 0.08$ & $0.88 \pm 0.08 \dagger$ & $0.94 \pm 0.06 \dagger \ddagger$ \\
\hline Sagittal diameter $(\mathrm{cm})$ & $19.6(17.9-23.1)$ & $19.5(17.8-21.8)$ & $22.8(21.0-25.1) \dagger \neq$ \\
\hline SAT-US, cm & $2.5 \pm 1.1$ & $2.5 \pm 1.2$ & $2.9 \pm 1.1 \dagger \neq$ \\
\hline VAT-US, cm & $6.7(5.6-9.0)$ & $6.9(5.5-8.6) \dagger$ & $8.4(7.3-10.5) \dagger \ddagger$ \\
\hline SKF biceps, mm & $8(6-11)$ & $9(6-14)$ & $11(7-17) \dagger$ \\
\hline SKF triceps, mm & $15(10-20)$ & $16(11-23)$ & $16(10-24)$ \\
\hline SKF subscapular, mm & $18(13-22)$ & $20(14-25)$ & $24(18-30) \dagger$ \\
\hline SKF supra-iliacal, mm & $22(16-32)$ & $25(16-32)$ & $26(22-31)$ \\
\hline $\begin{array}{l}\text { Fatty liver } \% \\
\text { (mild, moderate, severe) }\end{array}$ & $18(5,7,6)$ & $25(15,7,3)$ & $49(24,12,13) \dagger$ \\
\hline
\end{tabular}

Data are presented as mean \pm SD or as median (interquartile range)

$* \mathrm{p}<0.05$ in comparison with spouses and FCHL patients

$\dagger \mathrm{p}<0.05$ in comparison with spouses, age and sex adjusted

$\neq \mathrm{p}<0.05$ in comparison with NL relatives, age and sex adjusted

All analyses are Hochberg corrected

were repeated with further adjustment for obesity, measured as BMI, the prevelance of fatty liver remained elevated in FCHL patients in comparison with spouses (Odds ratio [OR]: 3.1; 95\%CI: $1.1-8.7 ; \mathrm{p}=0.03)$. Moreover, a significant difference in prevalence was also observed for NL relatives versus spouses (OR: 4.0; 95\%CI: $1.3-12.3$; $\mathrm{p}=0.02$ ), whereas the difference between FCHL patients and NL relatives was completely abolished after correction for BMI (OR: 0.8; 95\%CI: $0.3-1.9 ; \mathrm{p}=0.6$ ). Of note, very similar results were obtained when FCHL 
Table 2.

Univariate correlations of anthropometric parameters with fatty liver stages in FCHL family members

\begin{tabular}{lcc}
\hline & $\mathrm{r}^{2}$ & $\mathrm{p}$-value \\
\hline BMI & 0.44 & $<0.001$ \\
Waist circumference & 0.43 & $<0.001$ \\
Hip circumference & 0.24 & $<0.001$ \\
Waist to hip ratio & 0.27 & $<0.001$ \\
Sagittal diameter & 0.41 & $<0.001$ \\
VAT-US & 0.32 & $<0.001$ \\
SAT-US & 0.07 & 0.001 \\
Biceps SKF & 0.08 & $<0.001$ \\
Triceps SKF & 0.05 & 0.008 \\
Subscapular SKF & 0.28 & $<0.001$ \\
Supra-iliacal SKF & 0.07 & $<0.001$ \\
\hline
\end{tabular}

Analysed with ordinal regression

patients were defined according to recently proposed criteria of plasma triglycerides $>1.5$ $\mathrm{mmol} / \mathrm{L}$ and apo B levels $>1.2 \mathrm{~g} / \mathrm{L}$ [14] (OR: 4.2, $\mathrm{p}=0.02$ for FCHL patients vs spouses; OR: $4.7, \mathrm{p}=0.006$ for relatives vs spouses). In addition, similar trends were observed when only one FCHL patient or one NL relative from each family was taken for analysis (data not shown).

\section{Independent contribution of different fat compartments to fatty liver in FCHL patients and NL relatives}

In all FCHL family members, i.e. FCHL patients and NL relatives combined, all anthropometric variables were significantly correlated with the different stages of fatty liver in univariate analysis (Table 2 and Figure 1). Subsequently, age and sex corrected multivariate models were constructed to study the independent contribution of the different fat compartments to the fatty liver stages. As shown in Table 3, the prediction models that included either overall obesity, represented by BMI (model 1), or abdominal obesity (model 2), resulted in very similar correlation coefficients of 0.46 and 0.44 , respectively. A model with only visceral obesity, i.e. VAT-US measured at the same level as waist circumference, yielded a substantially lower coefficient (model 3). Of interest, when SAT-US was added to VAT-US (model 4a), the correlation coefficient very much approached the $\mathrm{r}^{2}$ of the model that included waist circumference (model 2). Replacing SAT-US by subscapular skinfold resulted in a similar model (model $4 \mathrm{~b}$ ), whereas biceps skinfold only marginally added to the contribution of VAT-US (model 4c versus model 3). Combining VATUS, subscapular and biceps skinfold into one model (model 5) showed that both visceral and subcutaneous obesity of the trunk, but not of the upper limb, independently contributed to the prediction of the different stages of fatty liver. Of note, correction for the hyperlipidemic state, i.e. being FCHL patient or normolipidemic relative (classical [1] or newly proposed [14] criteria), did not influence these results. 

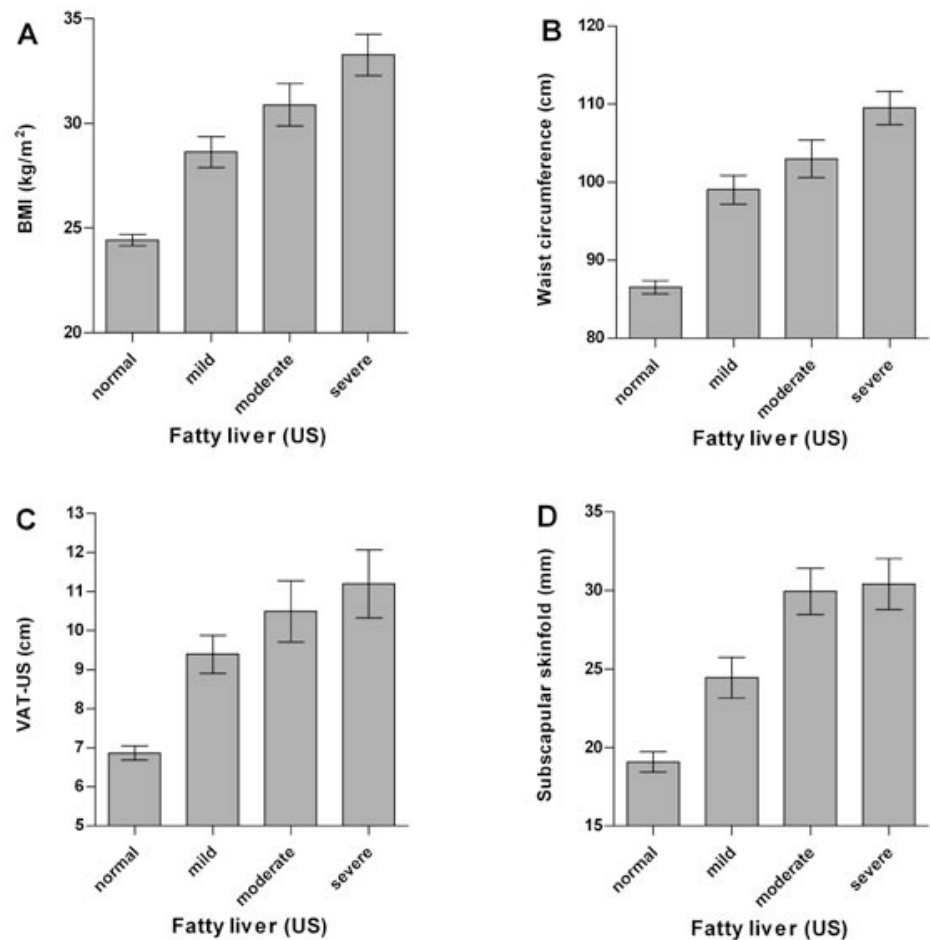

Figure 1.

Relationship of fatty liver stages with BMI (A), waist circumference (B), VAT-US (C) and subscapular SKF (D) in FCHL family members, i.e. patients and relatives combined (numbers of family members for each fatty liver stage: 120 normal, 53 mild, 16 moderate, 11 severe fatty liver). Error bars represent SEM

Relation of plasma triglycerides, apolipoprotein B and insulin levels with fatty liver stages in FCHL patients and normolipidemic relatives

In univariate, ordinal regression analysis, insulin levels were positively correlated with the different stages of fatty liver $\left(\mathrm{r}^{2}=0.32, \mathrm{p}<0.001\right.$, Figure 2$)$, independent of the affected state.

Of the two newly proposed parameters to diagnose FCHL [14], only plasma triglycerides were significantly related with the fatty liver stages $\left(r^{2}=0.22, p<0.001\right)$, whereas plasma apo B levels were not $\left(\mathrm{r}^{2}=0.002, \mathrm{p}=0.6\right)$.

Since plasma triglycerides are mainly secreted by the liver as VLDL-particles, we subsequently decided to measure VLDL-triglycerides and VLDL-apo B concentrations in a representative subset of relatives and patients $(n=69)$ and to relate these parameters with fatty liver. VLDLtriglycerides were similarly correlated with fatty liver stages as total plasma triglycerides $\left(\mathrm{r}^{2}=\right.$ $0.18, \mathrm{p}=0.001$, Figure 3, panel A). Of interest, VLDL-apo B did show a correlation with fatty liver in contrast to total plasma apo $\mathrm{B}\left(\mathrm{r}^{2}=0.12, \mathrm{p}=0.006\right.$, Figure 3 , panel $\left.\mathrm{B}\right)$. 
Table 3.

Prediction models of fatty liver stages with different fat compartments in FCHL family members

\begin{tabular}{|c|c|c|c|c|}
\hline Model & & Model $r^{2}$ & Variables entered & P-value \\
\hline 1. Overall obesity & & 0.46 & BMI & $<0.001$ \\
\hline 2. Abdominal obesity & & 0.44 & Waist circumference & $<0.001$ \\
\hline 3. Visceral obesity & & 0.35 & VAT-US & $<0.001$ \\
\hline \multirow[t]{3}{*}{$\begin{array}{l}\text { 4. Visceral + subcutaneous } \\
\text { obesity }\end{array}$} & a & 0.43 & $\begin{array}{l}\text { VAT-US } \\
\text { SAT-US }\end{array}$ & $\begin{array}{l}<0.001 \\
<0.001\end{array}$ \\
\hline & $\mathrm{b}$ & 0.40 & $\begin{array}{c}\text { VAT-US } \\
\text { Subscapular SKF }\end{array}$ & $\begin{array}{l}<0.001 \\
<0.001\end{array}$ \\
\hline & $\mathrm{c}$ & 0.36 & $\begin{array}{c}\text { VAT-US } \\
\text { Biceps SKF }\end{array}$ & $\begin{array}{c}<0.001 \\
0.01\end{array}$ \\
\hline $\begin{array}{l}\text { 5. Visceral }+ \text { subcutaneous } \\
\text { (limb }+ \text { trunk) obesity }\end{array}$ & & 0.41 & $\begin{array}{c}\text { VAT-US } \\
\text { Subscapular SKF } \\
\text { Biceps SKF }\end{array}$ & $\begin{array}{c}<0.001 \\
0.002 \\
0.20\end{array}$ \\
\hline
\end{tabular}

Analysed with ordinal regression with inclusion of age and sex as co-variates. Including FCHL status, i.e. being a FCHL or NL relative, as a co-variate did not influence the results

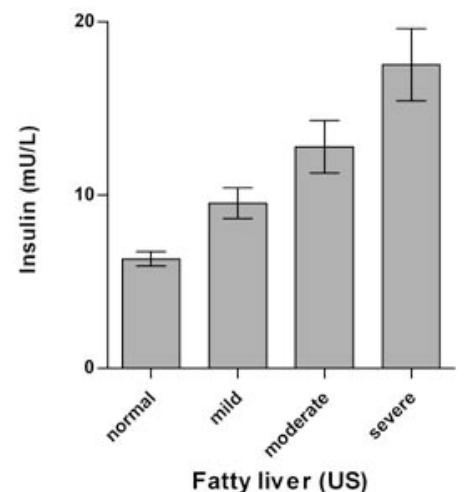

Figure 2.

Relation of fatty liver stages with plasma insulin levels in FCHL family members, i.e. patients and relatives combined. Error bars represent SEM 

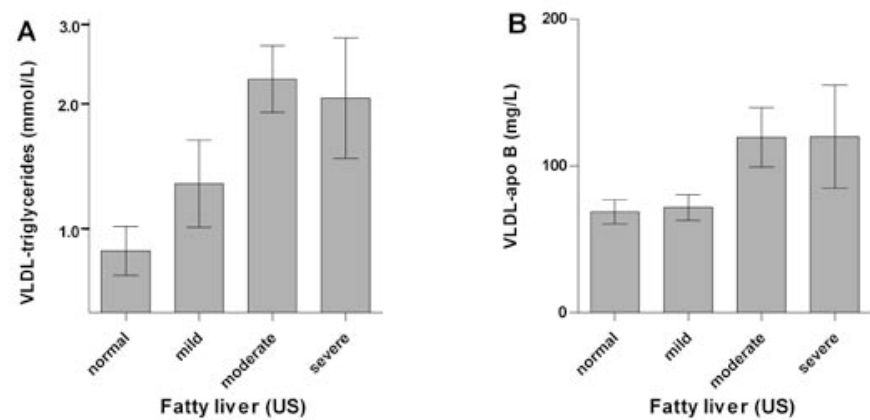

Figure 3.

Relation of fatty liver stages with VLDL-triglycerides (panel A) and VLDL-apo B (panel B) in a subset of FCHL family members $(n=69)$. Error bars represent SEM

\section{Discussion}

Our laboratory and others have previously demonstrated that familial combined hyperlipidemia develops against a background of abdominal obesity [7, 8]. The present study was conducted to relate these findings to fatty liver, a condition that is known to drive the production of VLDLparticles [12], which is an important feature of FCHL [9, 10].

The present study showed that both NL relatives and FCHL patients exhibited a three to four times increased risk (as expressed by the odds ratio) of having fatty liver in comparison with spouses. The prevalence of fatty liver was considerably higher in FCHL patients in comparison with spouses (49\% versus 18\%). This number seems to be somewhat lower than the previously reported prevalence of $76 \%$ in an outpatient FCHL population [13]. This discrepancy is likely to be explained by the higher co-morbidity that is often seen in outpatient populations in comparison with a non-outpatient population, as was predominantly the case in the present study. Furthermore, the previous study was conducted in a relatively small sample, thus yielding prevalence numbers with large confidence intervals. The prevalence estimate from the present larger population is therefore expected to be more reliable.

In all FCHL family members, obesity was an important contributor to the grade of fatty liver: $46 \%$ of the variation in fatty liver grade was accounted for by BMI. Of interest, a very similar correlation was observed for the abdominal compartment, quantified as waist circumference, indicating that it is specifically this region that predicts fatty liver in FCHL. A more detailed study in this region revealed that both the visceral as well as the subcutaneous fat depot, both measured with ultrasound, were independent predictors of fatty liver grade. Furthermore, the combination of these two compartments very much approached the model that included only 
waist circumference. The association of visceral fat with fatty liver has been demonstrated in several other conditions associated with the metabolic syndrome [23-25], whereas the correlation of the subcutaneous adipose tissue with fatty liver is more controversial [23, 24]. Nielsen et al. have elegantly shown that both visceral adipose tissue and subcutaneous adipose tissue contribute to the flux of free fatty acids to the liver [26, 27], which is thought to be the most important process that drives hepatic fat accumulation [28]. The present study also suggests that the independent contribution of the subcutaneous fat depot in FCHL is confined to the truncal region. Of interest, Abate et al. have demonstrated that subcutaneous truncal but not peripheral fat is associated with the hepatic glucose production [29], a process that is related with hepatic fat accumulation [23].

The importance of obesity in the relation with fatty liver was also illustrated by the observation that BMI completely abolished the seemingly marked difference in prevalence of fatty liver between FCHL patients and NL relatives (49\% versus 25\%). This indicates that FCHL patients and their NL relatives are very similar regarding the prevalence of fatty liver, with the only exception that the patients are more obese and older. In contrast, the almost threefold higher prevalence of fatty liver in FCHL patients in comparison with spouses remained significant after correction for age, gender and BMI. Additionally, the apparently small prevalence difference between NL relatives and spouses became significant after correction for age, gender and BMI. These data imply an increased susceptibility of FCHL family members in general for the development of fatty liver, independent of the affected state (defined by both the classical [1] and the more recently proposed [14] criteria). Genetic factors could account for this lower threshold, since FCHL family members and spouses share the same environment.

The central role of fatty liver in FCHL was further emphasized by its association with both insulin levels and plasma triglycerides. Apo B levels, another hallmark of FCHL, were not related with the different fatty liver stages. These results are in concordance with Toledo et al. who reported very similar outcomes in a diabetes population [30]. Of interest, in that report the relation of fatty liver with plasma triglycerides was mainly explained by VLDL-particle size [30], whereas our study suggested that VLDL-particle number, as reflected by VLDL-apo B levels, was associated with the different stages of fatty liver. It should be noted that an increase in VLDL-particle number could be due to an increased VLDL-production, but also to an impaired clearance.

The apparent discrepancy between our findings in FCHL and previous observations in type 2 diabetes mellitus [30] may be explained by the study populations that were under investigation. Previous studies have suggested that VLDL-particles are larger in patients type 2 diabetes mellitus, but of normal size in FCHL patients [31, 32].

In the present study, we used liver ultrasound to detect hepatic fat accumulation. The gold standard, liver biopsy, was not ethically accepted in this relatively healthy population. Liver ultrasound was therefore used as an alternative, validated method to detect hepatic fat accumulation $[18,19]$. Since ultrasound is less sensitive to detect mild stages of fatty liver [18], it is anticipated that the 
presently observed prevalence of fatty liver in FCHL is somewhat underestimated. Of note, the prevalence of fatty liver in the presently studied spouses was similar to the prevalence of fatty liver detected by histology in a general population [33, 34].

In summary, we showed that not only FCHL patients, but also their normolipidemic relatives have a three times higher risk of developing fatty liver compared to spouses. Within the FCHL population, BMI is an important contributor to fatty liver grade, and both the subcutaneous truncal and the visceral fat depots independently contributed to this association. The relation of fatty liver with plasma insulin, VLDL-triglycerides and VLDL-apo B levels further underlines the central role of fatty liver in the pathogenesis and expression of FCHL.

\section{References}

[1] Goldstein JL, Schrott HG, Hazzard WR, Bierman EL, Motulsky AG (1973) Hyperlipidemia in coronary heart disease. II. Genetic analysis of lipid levels in 176 families and delineation of a new inherited disorder, combined hyperlipidemia. J Clin Invest 52: 1544-1568

[2] Ayyobi AF, McGladdery SH, McNeely MJ, Austin MA, Motulsky AG, Brunzell JD (2003) Small, Dense LDL and Elevated Apolipoprotein B Are the Common Characteristics for the Three Major Lipid Phenotypes of Familial Combined Hyperlipidemia. Arterioscler Thromb Vasc Biol 23: 1289-1294

[3] Veerkamp MJ, de Graaf J, Bredie SJ, Hendriks JC, Demacker PN, Stalenhoef AF (2002) Diagnosis of familial combined hyperlipidemia based on lipid phenotype expression in 32 families: results of a 5-year follow-up study. Arterioscler Thromb Vasc Biol 22: 274-282

[4] Aitman TJ, Godsland IF, Farren B, Crook D, Wong HJ, Scott J (1997) Defects of insulin action on fatty acid and carbohydrate metabolism in familial combined hyperlipidemia. Arterioscler Thromb Vasc Biol 17: 748-754

[5] Carr MC, Brunzell JD (2004) Abdominal obesity and dyslipidemia in the metabolic syndrome: importance of type 2 diabetes and familial combined hyperlipidemia in coronary artery disease risk. J Clin Endocrinol Metab 89: 2601-2607

[6] Purnell JQ, Kahn SE, Schwartz RS, Brunzell JD (2001) Relationship of insulin sensitivity and ApoB levels to intra-abdominal fat in subjects with familial combined hyperlipidemia. Arterioscler Thromb Vasc Biol 21: 567-572

[7] van der Kallen CJ, Voors-Pette C, de Bruin TW (2004) Abdominal obesity and expression of familial combined hyperlipidemia. Obes Res 12: 2054-2061

[8] Ascaso JF, Sales J, Merchante A, et al. (1997) Influence of obesity on plasma lipoproteins, glycaemia and insulinaemia in patients with familial combined hyperlipidaemia. Int J Obes Relat Metab Disord 21: 360-366

[9] Venkatesan S, Cullen P, Pacy P, Halliday D, Scott J (1993) Stable isotopes show a direct relation between VLDL apoB overproduction and serum triglyceride levels and indicate a metabolically and biochemically coherent basis for familial combined hyperlipidemia. Arterioscler Thromb 13: 1110-1118 
[10] Kissebah AH, Alfarsi S, Adams PW (1981) Integrated regulation of very low density lipoprotein triglyceride and apolipoprotein-B kinetics in man: normolipemic subjects, familial hypertriglyceridemia and familial combined hyperlipidemia. Metabolism 30: 856 868

[11] Cabezas MC, de Bruin TW, Jansen H, Kock LA, Kortlandt W, Erkelens DW (1993) Impaired chylomicron remnant clearance in familial combined hyperlipidemia. Arterioscler Thromb 13: 804-814

[12] Adiels M, Taskinen MR, Packard C, et al. (2006) Overproduction of large VLDL particles is driven by increased liver fat content in man. Diabetologia: 1-11

[13] de Bruin TW, Georgieva AM, Brouwers MC, Heitink MV, van der Kallen CJ, van Greevenbroek MM (2004) Radiological evidence of nonalcoholic fatty liver disease in familial combined hyperlipidemia. Am J Med 116: 847-849

[14] Sniderman AD, Castro Cabezas M, Ribalta J, et al. (2002) A proposal to redefine familial combined hyperlipidaemia -- third workshop on FCHL held in Barcelona from 3 to 5 May 2001, during the scientific sessions of the European Society for Clinical Investigation. Eur J Clin Invest 32: 71-73

[15] Keulen ET, Voors-Pette C, de Bruin TW (2001) Familial dyslipidemic hypertension syndrome: familial combined hyperlipidemia, and the role of abdominal fat mass. Am J Hypertens 14: 357-363

[16] Farrell GC (2002) Drugs and steatohepatitis. Semin Liver Dis 22: 185-194

[17] Stolk RP, Wink O, Zelissen PM, Meijer R, van Gils AP, Grobbee DE (2001) Validity and reproducibility of ultrasonography for the measurement of intra-abdominal adipose tissue. Int J Obes Relat Metab Disord 25: 1346-1351

[18] Saadeh S, Younossi ZM, Remer EM, et al. (2002) The utility of radiological imaging in nonalcoholic fatty liver disease. Gastroenterology 123: 745-750

[19] Fishbein M, Castro F, Cheruku S, et al. (2005) Hepatic MRI for Fat Quantitation: Its Relationship to Fat Morphology, Diagnosis, and Ultrasound. J Clin Gastroenterol 39: 619-625

[20] Keulen ET, Kruijshoop M, Schaper NC, Hoeks AP, de Bruin TW (2002) Increased intima media thickness in familial combined hyperlipidemia associated with apolipoprotein B. Arterioscler Thromb Vasc Biol 22: 283-288

[21] Redgrave TG, Roberts DC, West CE (1975) Separation of plasma lipoproteins by density gradient ultracentrifugation. Anal Biochem 65: 42-49

[22] Karpe F, Hamsten A (1994) Determination of apolipoproteins B-48 and B-100 in triglyceride-rich lipoproteins by analytical SDS-PAGE. J Lipid Res 35: 1311-1317

[23] Kelley DE, McKolanis TM, Hegazi RA, Kuller LH, Kalhan SC (2003) Fatty liver in type 2 diabetes mellitus: relation to regional adiposity, fatty acids, and insulin resistance. Am J Physiol Endocrinol Metab 285: E906-916

[24] Westerbacka J, Corner A, Tiikkainen M, et al. (2004) Women and men have similar amounts of liver and intra-abdominal fat, despite more subcutaneous fat in women implications for sex differences in markers of cardiovascular risk. Diabetologia 47: 1360-1369 
[25] Knobler H, Schattner A, Zhornicki T, et al. (1999) Fatty liver--an additional and treatable feature of the insulin resistance syndrome. Qjm 92: 73-79

[26] Klein S (2004) The case of visceral fat: argument for the defense. J Clin Invest 113: $1530-1532$

[27] Nielsen S, Guo Z, Johnson CM, Hensrud DD, Jensen MD (2004) Splanchnic lipolysis in human obesity. J Clin Invest 113: 1582-1588

[28] Donnelly KL, Smith CI, Schwarzenberg SJ, Jessurun J, Boldt MD, Parks EJ (2005) Sources of fatty acids stored in liver and secreted via lipoproteins in patients with nonalcoholic fatty liver disease. J Clin Invest 115: 1343-1351

[29] Abate N, Garg A, Peshock RM, Stray-Gundersen J, Grundy SM (1995) Relationships of generalized and regional adiposity to insulin sensitivity in men. J Clin Invest 96: 88-98

[30] Toledo FG, Sniderman AD, Kelley DE (2006) Influence of hepatic steatosis (Fatty liver) on severity and composition of dyslipidemia in type 2 diabetes. Diabetes Care 29: $1845-1850$

[31] McEneny J, O’Kane MJ, Moles KW, et al. (2000) Very low density lipoprotein subfractions in Type II diabetes mellitus: alterations in composition and susceptibility to oxidation. Diabetologia 43: 485-493

[32] van Barlingen HH, Kock LA, de Man FH, Erkelens DW, de Bruin TW (1996) In vitro lipolysis of human VLDL: effect of different VLDL compositions in normolipidemia, familial combined hyperlipidemia and familial hypertriglyceridemia. Atherosclerosis 121: $75-84$

[33] Underwood Ground KE (1984) Prevalence of fatty liver in healthy male adults accidentally killed. Aviat Space Environ Med 55: 59-61

[34] Hilden M, Christoffersen P, Juhl E, Dalgaard JB (1977) Liver histology in a 'normal' population--examinations of 503 consecutive fatal traffic casualties. Scand J

Gastroenterol 12: 593-597 
Genetic predisposition to develop fatty liver

Five-year follow-up of waist circumference, insulin and ALT levels in familial combined hyperlipidemia

Brouwers MC, van Greevenbroek MM Vermeulen VM, van Lin JM van der Kallen CJ de Bruin TW

Clin Sci (Lond). 2007; 113(9): 375-381 


\begin{abstract}
Familial combined hyperlipidemia (FCHL), an entity with many features of the metabolic syndrome, is characterized by changes in cholesterol and triglyceride phenotype over time. This study was conducted to investigate the relation of alanine aminotransferase (ALT) levels, used as a surrogate for the amount of hepatic fat, with the switch in triglyceride phenotype and the increased susceptibility to develop hypertriglyceridemia in FCHL.

Body mass index, waist circumference, plasma triglycerides, insulin and ALT levels were measured in 145 FCHL family members and 54 spouses at baseline and after a five-year follow-up.

A switch from normotriglyceridemia to hypertriglyceridemia or vice versa, as observed in 22 of 145 FCHL family members, was associated with changes in plasma ALT levels $(p=0.001)$, but not with insulin levels or waist circumference. In five-year follow-up, an intra-individual relation was observed between waist circumference and both plasma triglycerides, insulin and ALT levels. For each waist circumference FCHL patients, but not their normolipidemic relatives, exhibited higher triglyceride and insulin levels than spouses $(\mathrm{p}<0.001)$. Remarkably, both FCHL patients and the normolipidemic relatives showed higher ALT levels for each waist circumference as compared to spouses ( $p<0.001$ for FCHL patients, $p=0.035$ for normolipidemic relatives). The present study shows that the longitudinal relation abdominal obesity-ALT is more specific for all FCHL family members, i.e. patients and their normolipidemic relatives, than the relation abdominal obesity-triglycerides. Additionally, the association of ALT with the switch in triglyceride phenotype suggests a central role of the liver in the pathogenesis of FCHL.
\end{abstract}




\section{Introduction}

Familial Combined Hyperlipidemia (FCHL) is a common primary hyperlipidemia characterized by premature cardiovascular disease [1] and features of the metabolic syndrome, such as insulin resistance [2], abdominal obesity [3] and hypertension [4]. FCHL is, by definition, a 'multiple-type hyperlipidemia', in which different Frederickson's subtypes of hyperlipidemia are present within one family (IIa, IIb and IV, corresponding with isolated hypercholesterolemia, combined hyperlipidemia and isolated hypertriglyceridemia, respectively) [1]. Shortly after the first description of FCHL, it was suggested that the 'multiple-type hyperlipidemia' could occur within one particular FCHL patient as well, and that expression of a hyperlipidemic phenotype at a certain point in time may be due to the combination of genetic and environmental factors [5]. The 'multiple-type hyperlipidemia' within one family makes the clinical diagnosis of FCHL demanding, given the necessity to screen for hyperlipidemia in at least two family members. Furthermore, the 'multiple-type hyperlipidemia' within one individual may lead to misclassification of the affected state, since subjects may be hyperlipidemic at one time-point, but normolipidemic at another measurement. Efforts have therefore been made to simplify and standardize the diagnosis of the FCHL phenotype, among others by including markers that are less variable over time, such as plasma apolipoprotein B levels $[6,7]$.

The hypertriglyceridemic phenotype in FCHL is the consequence of both an excessive production of VLDL-particles and an impaired clearance of remnants [8-10]. The former has been associated with both hepatic insulin resistance and hepatic fat accumulation [11, 12], which makes them good candidates for explaining the multiple-type hyperlipidemia in FCHL. Indeed, insulin resistance has been associated with the switch in triglyceride phenotype, i.e. from normotriglyceridemia to hypertriglyceridemia or vice versa, in FCHL [13]. To date, the relation between hepatic fat accumulation and the multiple-type hyperlipidemia in FCHL has been unknown. This relation is conceivable given the high prevalence of fatty liver in particularly hypertriglyceridemic FCHL patients [14, 15].

Therefore, the present study was conducted to investigate whether varying amounts of hepatic fat are responsible for the 'multiple-type hyperlipidemia' in FCHL, in particular the switch in triglyceride phenotype. Since an appreciable proportion of hepatic fat is derived from free fatty acids released from adipose tissue [16], we additionally questioned how the degree of (abdominal) obesity is related to fatty liver, insulin resistance and plasma triglycerides in individual FCHL patients, their normolipidemic relatives and spouses.

For this alanine aminotransferase (ALT) levels were determined with a five-year interval and evaluated in relation to lipid, insulin and obesity parameters in 21 FCHL pedigrees and their spouses. This liver specific enzyme is correlated with liver fat in normal subjects [17] and also in FCHL families [18], and has often been used in longitudinal studies as a marker of nonalcoholic fatty liver disease [19-21], a histological spectrum that comprises hepatic steatosis [22]. 


\section{Materials and methods}

\section{Study population}

FCHL families were ascertained as described previously [23]. Briefly, the diagnosis was established when at least two family members exhibited different types of hyperlipidemia, i.e. hypertrigycleridemia (triglycerides $>2.3 \mathrm{mmol} / \mathrm{L}$ ), hypercholesterolemia (cholesterol $>6.5$ $\mathrm{mmol} / \mathrm{L}$ ) or both, the so-called multiple-type hyperlipidemia. Furthermore, at least one family member had to have a history of premature cardiovascular disease. Exclusion criteria were other genetic hyperlipidemias, such as familial hypercholesterolemia and familial dysbetalipoproteinemia (E2/E2 genotype). FCHL probands, i.e. the index patients from each family, were non-obese (body mass index $<30 \mathrm{~kg} / \mathrm{m}^{2}$ ), non-diabetic and had a normal thyroid function. Recently, it was proposed to redefine FCHL according to factors that are more specific for FCHL and constant over time, i.e. apolipolipoprotein B $>1.2 \mathrm{~g} / \mathrm{L}$ and triglycerides $>1.5 \mathrm{mmol} / \mathrm{L}[6]$. In the current study, all analyses are conducted with both the traditional and newly proposed criteria of FCHL to assess the impact of these different criteria on the outcomes.

FCHL patients (i.e. all hyperlipidemic members of the established FCHL families), their normolipidemic (NL) relatives (i.e. all normolipidemic members of the established FCHL families) and their spouses visited the lipid research clinic after an overnight fast and complete abstinence from alcohol for the last three days. Lipid-lowering medication was withdrawn two weeks prior to their visit. Additionally, none of the subjects reported rapid weight loss, took any medication associated with the development of fatty liver [24] or consumed more than two units of alcohol daily. One subject was found to be seropositive for hepatitis C and was therefore excluded from this study. All subjects filled in a questionnaire regarding smoking and dietary habits.

In 2004, subjects were re-recruited in the same order as in 1999, thereby preventing a difference in follow-up period for different subgroups. In total, 21 families, consisting of 199 subjects (FCHL family members and spouses), were studied in both 1999 (baseline) and 2004. Nine of $63(14 \%)$ spouses who were invited for the second visit were lost to follow-up. Three subjects deceased in the follow-up period, the remaining six rejected further participation. They were significantly older than the 54 spouses with a follow-up measurement $(75$ versus 47 years, $\mathrm{p}<$ 0.001). There were no differences in age adjusted baseline cholesterol, triglycerides and body mass index (BMI) values. Of all 195 FCHL family members who were invited, 50 subjects $(26 \%)$ were lost in follow-up because of death $(n=8)$ or reluctance to participate $(n=42)$. Their baseline age, sex, cholesterol, triglyceride and BMI values were not different from the FCHL family members with a follow-up measurement.

All subjects gave written informed consent. The research protocol was approved by the medical ethical review committee at the Academic Hospital Maastricht and Maastricht University. 


\section{Anthropometric measurements}

Height was measured with a stadiometer. Weight was determined in underwear. Body mass index (BMI) was calculated as weight divided by height squared $\left(\mathrm{kg} / \mathrm{m}^{2}\right)$. Waist circumference was measured in standing position at the level of the umbilicus.

\section{Laboratory measurements}

Plasma cholesterol, triglycerides, insulin, apolipoprotein B, glucose and ALT determinations were done as described previously [4, 18]. HOMA-IR (homeotastasis model assessment insulin resistance) was calculated as a measure of insulin resistance [25].

\section{Statistical methods}

Data are expressed as mean \pm standard deviation (SD), or as median with interquartile range between brackets in case of non-normal distribution. An one-way ANOVA was used to detect age differences between FCHL patients, their NL relatives and spouses. Other variables were tested in linear regression models with inclusion of age and sex.

Prevalences of hyperlipidemia and changing phenotype between FCHL families and spouses were compared with a chi-square test. Waist circumference, plasma insulin and ALT levels in 1999 and 2004 were compared with the non-parametric Wilcoxon test for related samples. A mixed linear model for repeated measurements with random intercept was constructed to test the five-year longitudinal relations between waist circumference, ALT, insulin and triglyceride levels. Since the repeated measurements within one individual were included in the model as a random factor, the outcome of the model should be interpreted as the intra-individual relation averaged for all subjects. Obviously, such an intra-individual relation is superior to the inter-individual relation that is normally obtained in cross-sectional study designs. Dummy variables were entered in these models to study differences in these longitudinal relations between spouses and FCHL patients (spouse $=0$ and FCHL patient $=1$ ), and between spouses and normolipidemic relatives ( spouse $=0$ and NL relative $=1)$. All analyses were conducted with SPSS 13.0 statistical package (SPSS Inc. Illinois, USA).

\section{Results}

\section{Baseline characteristics}

Of all 199 subjects who were studied, 54 were spouses and 145 were FCHL family members derived from 21 FCHL families. At baseline (in 1999), 56 of 145 (39\%) subjects fulfilled the traditional FCHL criteria of hyperlipidemia, 29 (52\%) were hypercholesterolemic, 15 (27\%) were hypertriglyceridemic and $12(21 \%)$ were combined hyperlipidemic. The descriptives of these hyperlipidemic patients, their NL relatives and their spouses are listed in Table 1. FCHL patients were older than their NL relatives, but not in comparison with their spouses $(p<0.001$, one-way ANOVA). After correction for age and sex, FCHL patients were significantly more (abdominally) obese, and had higher insulin and ALT levels than their NL relatives and spouses (Table 1). 
Table 1.

Baseline characteristics of FCHL patiens, NL relatives and spouses

\begin{tabular}{lccc}
\hline & Spouses & NL relatives & FCHL \\
\hline Male / Female & $27 / 27$ & $33 / 56$ & $27 / 29$ \\
Age, years & $47 \pm 10$ & $39 \pm 16^{*}$ & $50 \pm 13$ \\
BMI, kg/m2 & $25.5 \pm 3.7$ & $25.2 \pm 4.1$ & $28.1 \pm 3.9+\ddagger$ \\
Waist circumference, cm & $90.2 \pm 10.5$ & $88.8 \pm 12.8$ & $96.5 \pm 10.5+\ddagger$ \\
Cholesterol, mmol/L & $5.2 \pm 0.8$ & $5.0 \pm 0.9$ & $6.7 \pm 1.2 \dagger \neq$ \\
Triglycerides, mmol/L & $1.1(0.7-1.6)$ & $1.1(0.9-1.4)$ & $2.0(1.4-2.6) \dagger \ddagger$ \\
Apolipoprotein B, g/L & $1.0 \pm 0.2$ & $1.0 \pm 0.2$ & $1.4 \pm 0.3 \dagger \neq$ \\
Insulin, mU/L & $4.9(2.0-9.1)$ & $5.4(2.0-8.9)$ & $8.2(5.2-13.5) \dagger \ddagger$ \\
ALT, U/L & $14.8(11.9-19.4)$ & $15.9(11.5-20.9)$ & $24.4(18.3-31.1) \dagger \ddagger$ \\
\end{tabular}

Values are expressed as mean $\pm \mathrm{SD}$, or median (interquartile range). The affected state is defined according the traditional criteria.

${ }^{*} \mathrm{p}<0.001$ versus spouses and FCHL; student's T-test

$t p<0.05$ versus spouses after correction for age and sex; linear regression,

$\neq \mathrm{p}<0.05$ versus normolipidemic relatives after correction for age and sex; linear regression

All analyses were Hochberg corrected

\section{Changes in lipoprotein phenotype expression in 5-year follow-up}

At the second visit in 2004, again 56 of 145 FCHL family members fulfilled the FCHL criteria with an exactly similar distribution among the different phenotypes, but most of these were not the same individuals as at baseline: 43 of $145(30 \%)$ subjects switched lipid phenotype in the follow-up period (Table 2). Twenty-six subjects switched phenotype according to the cholesterol level and 22 subjects switched phenotype according to the triglyceride level (Table 2). In total, 32 of 145 subjects (22\%) switched from normolipidemia to FCHL or vice versa (Table 2). When FCHL was defined according to recently proposed criteria (apolipoprotein B > $1.2 \mathrm{~g} / \mathrm{L}$ and triglycerides $>1.5 \mathrm{mmol} / \mathrm{L}$ ) [6], the switch from affected to non-affected (or vice versa) was somewhat lower, i.e. 28 of 145 subjects (19\%). Only one of these subjects reported a beneficial change in dietary habits, which was accompanied by a switch from hyperlipidemia to normolipidemia. Eight of these subjects changed their smoking habits over time, which did not appear to be associated with the expression of the affected state.

At baseline, 7 of $54(13 \%)$ spouses exhibited the hyperlipidemic phenotype. As expected, this was significantly lower than the 56 of $145(39 \%)$ observed at baseline in the FCHL families ( $p$ $=0.001$, chi-square). At the second visit, 12 of $54(22 \%)$ spouses had switched lipid phenotype. However, this proportion was not significantly different from FCHL family members $30 \%$ in FCHL versus $22 \%$ in spouses, $\mathrm{p}=0.30$, chi-square). 
Table 2.

Lipid phenotype expression in FCHL in 1999 and 2004

\begin{tabular}{|c|c|c|c|c|c|c|}
\hline & & \multicolumn{5}{|c|}{2004} \\
\hline & & NL & $\mathrm{HC}$ & $\mathrm{HC}+\mathrm{HTG}$ & HTG & Total \\
\hline \multirow[t]{5}{*}{1999} & NL & 73 & 9 & 2 & 5 & 89 \\
\hline & $\mathrm{HC}$ & 8 & 17 & 3 & 1 & 29 \\
\hline & $\mathrm{HC}+\mathrm{HTG}$ & 2 & 3 & 5 & 2 & 12 \\
\hline & HTG & 6 & 0 & 2 & 7 & 15 \\
\hline & Total & 89 & 29 & 12 & 15 & 145 \\
\hline
\end{tabular}

Values represent absolute numbers of subjects

\section{Changes in triglyceride phenotype expression in relation with waist circumference, insulin and ALT levels}

The switch in triglyceride phenotype, as observed in 22 FCHL patients, was not associated with changes in waist circumference or plasma insulin levels (Figure 1, panel A and B). Similar results were obtained when BMI or HOMA levels were taken for analysis (data not shown). In contrast, ALT levels were significantly related with the switch in triglyceride phenotype between 1999 and 2004 ( $p=0.001$, Figure 1, panel C). This relation remained significant after correction for waist circumference and plasma insulin levels $(p=0.001)$. Of note, similar results were obtained when these variables were related to the change from normolipidemia to FCHL (or vice versa), which was defined according to the recently proposed consensus, i.e. apolipoprotein B $>1.2 \mathrm{~g} / \mathrm{L}$ and triglycerides $>1.5 \mathrm{mmol} / \mathrm{L}(\mathrm{p}=0.4$ for waist circumference, $\mathrm{p}=0.6$ for plasma insulin and $\mathrm{p}=$ 0.04 for ALT levels).
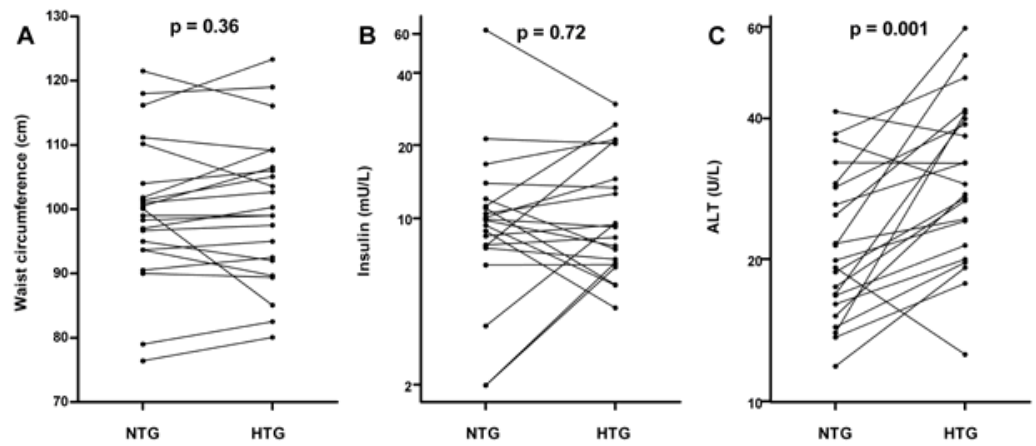

Figure 1.

Waist circumference, plasma insulin and alanine aminotransferase (ALT) levels in relation with the triglyceride phenotype switch (NTG: normotriglyceridemia, HTG: hypertriglyceridemia). Calculated with a Wilcoxon non-parametric test for related samples. Data are presented on a log scale 
Relation of abdominal obesity with plasma triglycerides, insulin and ALT levels in FCHL patients, NL relatives and spouses

Since the hyperlipidemic phenotype develops against a background of (abdominal) obesity [26], we subsequently investigated the intra-individual relation between abdominal obesity and plasma triglycerides, insulin and ALT levels in FCHL patients, normolipidemic relatives and spouses.

Despite the absent relation between waist circumference and the switch in triglyceride phenotype, an intra-individual relation was observed between plasma triglycerides and waist circumference in both spouses and FCHL family members (FCHL patients and NL relatives combined). Of interest, the FCHL family members were more susceptible to higher plasma triglycerides than spouses $(\mathrm{p}=0.004)$, which was explained by the FCHL patients, i.e. subjects who were hyperlipidemic in 1999 and/or 2004, as shown in Figure 2, panel A. For every waist circumference, FCHL patients had higher triglyceride levels than their spouses $(p<0.001)$. No differences were observed between spouses and the normolipidemic relatives, i.e. subjects who were normolipidemic in 1999 and 2004 ( $p=0.90$, Figure 2, panel A). Similar results were obtained for the intra-individual relation between abdominal obesity and plasma insulin levels (Figure 2, panel B). To investigate the role of the liver in this increased susceptibility, the relation between changes in waist circumference and changes in plasma ALT levels was subsequently examined. A five-year intra-individual relation was observed between waist circumference and ALT levels, which was again different between FCHL family members and spouses $(p<0.001)$. However, this time the difference was not only explained by FCHL patients, but also by the NL relatives, as shown in Figure 2, panel C. For every waist circumference, both NL relatives and FCHL patients exhibited significantly higher ALT levels than their spouses $(p=0.035$ and $p<$ 0.001, respectively). These outcomes were not affected by redefining FCHL according to the newly proposed criteria ( $\mathrm{p}=0.048$ and $\mathrm{p}<0.001$, respectively). Replacing waist circumference by BMI and insulin by HOMA-IR yielded very similar results (data not shown).
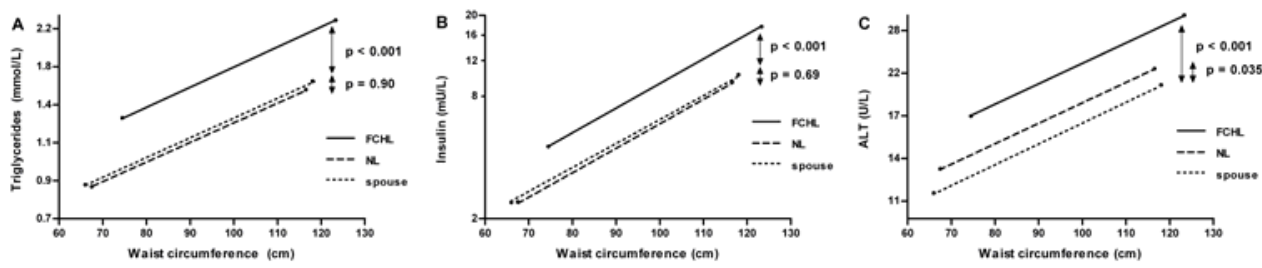

Figure 2.

5-year intra-individual relation between waist circumference and plasma triglycerides (panel A), insulin (panel B) and ALT levels (panel C) averaged for all spouses, NL relatives and FCHL patients. Regression lines, drawn from the minimum to the maximum waist value of each group, are the resultant of all individual five-year relations. Calculated with mixed linear regression for repeated measurements, age and gender corrected. Triglycerides, insulin and ALT are presented on a log scale. 


\section{Discussion}

The present study is the third to present long-term follow-up results in familial combined hyperlipidemia, and the first to incorporate data to evaluate the role of the liver in the pathogenesis of this highly prevalent genetic hyperlipidemia that is associated with premature cardiovascular complications. Longitudinal study designs are essential to study the changes in lipid phenotype over time, the so-called 'multiple-type hyperlipidemia' within one individual, a phenomenon that complicates the diagnosis and elucidation of FCHL.

In the current study, we observed that 43 of the 145 (30\%) FCHL family members had a different lipoprotein profile in 2004 in comparison with their initial measurement in 1999. This is in line with the 28 and 34\% reported in the two previous long-term follow-up studies in FCHL [27, 28]. However, this proportion was not significantly different from the $22 \%$ of spouses who changed lipid phenotype as well. These data suggest that the 'multiple-type hyperlipidemia' within one individual can occur in other conditions besides FCHL as well. Although this conclusion warrants some caution because of the small sample of controls that was used in the present study, it is of interest that Delawi et al. came to similar conclusions in a short-term follow-up study in FCHL patients and controls [29].

The current study focused on factors that are associated with the switch in lipid phenotype in FCHL. We anticipated that a switch in triglyceride phenotype is associated with changes in hepatic fat accumulation. This hypothesis was driven by a recent study reporting that the production of triglyceride-rich VLDL particles is positively associated with the amount of fat in the liver in both normal subjects and patients with type 2 diabetes mellitus [12]. Since liver biopsies were not ethically acceptable in this relatively healthy population, and magnetic resonance spectroscopy (MRS) was not widely in use at the initiation of this study, we determined plasma ALT levels as a marker of nonalcoholic fatty liver disease to test our hypothesis. ALT levels are correlated with liver fat quantified with ultrasound and MRS in normal subjects and FCHL families $[17,18]$, and have often been implemented as a surrogate for nonalcoholic fatty liver disease in longitudinal studies [19-21]. We found that ALT levels are indeed associated with the switch in triglyceride phenotype, suggestive of changes in hepatic fat as the driving force behind the change in triglyceride phenotype in FCHL. Of note, Westerbacka and colleagues have demonstrated that hepatic fat accumulation can indeed be a very reversible phenomenon [30]. Other factors that have previously been associated with the switch in triglyceride phenotype, i.e. obesity and insulin resistance [13,27], were not associated in the current study. This discrepancy may be explained by a lack of power. The currently observed significant intra-individual relation between (abdominal) obesity and plasma triglycerides in all FCHL patients seems to corroborate that suggestion. Alternatively, since plasma insulin levels reflect insulin resistance of liver, muscle as well as adipose tissue, they may not be a very specific marker for hepatic insulin resistance, which is another cause of VLDL-overproduction [11]. Furthermore, the effects of (abdominal) obesity on the hypertriglyceridemic phenotype are probably mediated through the induction of hepatic steatosis $[12,31]$. However, obesity is not a prerequisite for the development of 
hepatic fat accumulation [30]. The present study therefore shows that of the three markers under investigation, plasma ALT levels are most closely related to the expression of the hypertriglyceridemic phenotype in FCHL.

The potential role of the liver has also been implicated in the pathogenesis of other entities associated with the metabolic syndrome. Several groups have shown that ALT levels, even within the normal range, predict new-onset type 2 diabetes mellitus, and it has been proposed that ALT measurements, an easily obtainable biomarker, could be included in future diabetes prediction algorithms $[20,32]$. The present study suggests that the same could be true for the prediction of hypertriglyceridemia in FCHL.

The current results are in line with recent data published by Ter Avest et al. [33]. These authors concluded that elevated apolipoprotein B levels are the consequence of both a primary defect, possibly located in the liver, and of a secondary defect, which could be due to an increased hepatic free fatty acid flux resulting from expansion of adipose tissue [33]. The present study showed that hepatic fat accumulation, which drives the production of apolipoprotein B containing particles $[12,15]$, is also dependent on increments in abdominal obesity. In addition, with similar increments in abdominal obesity, not only FCHL patients, but also normolipidemic relatives exhibited significantly higher ALT levels, which is suggestive of an increased genetic susceptibility to develop fatty liver. Indeed, a genetic component was recently demonstrated in the same cohort [18].

An increased (genetic) susceptibility to develop fatty liver could have its origin in different processes, among others by an increased flux of free fatty acids towards the liver [16]. Of interest, Karjalainen et al. previously concluded that an excess hepatic supply of fatty acids, due to an increased release by adipose tissue in both FCHL patients and normolipidemic relatives, could precede the hyperlipidemia in FCHL [34]. These results are very much in concordance with our current data.

Unfortunately, in the present study we were not able to differentiate between different abdominal fat depots with distinctive features, i.e. visceral and subcutaneous obesity. This, together with the use of ALT as a surrogate of hepatic fat, should be regarded as a limitation of this study.

In summary, the present 5-year longitudinal study has underlined the role of the liver in the pathogenesis of FCHL by showing that the switch in triglyceride phenotype is associated with changes in ALT levels and that both FCHL patients and normolipidemic relatives exhibit higher ALT levels with similar increments in visceral obesity. Therefore, genes and metabolic pathways that contribute to the increased susceptibility to develop fatty liver in FCHL deserve our further attention. This will eventually lead to a better understanding of this highly prevalent disease characterized by different types of hyperlipidemia. 


\section{References}

[1] Goldstein JL, Schrott HG, Hazzard WR, Bierman EL, Motulsky AG (1973)

Hyperlipidemia in coronary heart disease. II. Genetic analysis of lipid levels in 176 families and delineation of a new inherited disorder, combined hyperlipidemia. J Clin Invest 52: 1544-1568

[2] Aitman TJ, Godsland IF, Farren B, Crook D, Wong HJ, Scott J (1997) Defects of insulin action on fatty acid and carbohydrate metabolism in familial combined hyperlipidemia. Arterioscler Thromb Vasc Biol 17: 748-754

[3] Purnell JQ, Kahn SE, Schwartz RS, Brunzell JD (2001) Relationship of insulin sensitivity and ApoB levels to intra-abdominal fat in subjects with familial combined hyperlipidemia. Arterioscler Thromb Vasc Biol 21: 567-572

[4] Keulen ET, Voors-Pette C, de Bruin TW (2001) Familial dyslipidemic hypertension syndrome: familial combined hyperlipidemia, and the role of abdominal fat mass. Am J Hypertens 14: 357-363

[5] Brunzell JD, Albers JJ, Chait A, Grundy SM, Groszek E, McDonald GB (1983) Plasma lipoproteins in familial combined hyperlipidemia and monogenic familial hypertriglyceridemia. J Lipid Res 24: 147-155

[6] Sniderman AD, Castro Cabezas M, Ribalta J, et al. (2002) A proposal to redefine familial combined hyperlipidaemia -- third workshop on FCHL held in Barcelona from 3 to 5 May 2001, during the scientific sessions of the European Society for Clinical Investigation. Eur J Clin Invest 32: 71-73

[7] Veerkamp MJ, de Graaf J, Hendriks JC, Demacker PN, Stalenhoef AF (2004) Nomogram to diagnose familial combined hyperlipidemia on the basis of results of a 5-year follow up study. Circulation 109: 2980-2985

[8] Kissebah AH, Alfarsi S, Adams PW (1981) Integrated regulation of very low density lipoprotein triglyceride and apolipoprotein-B kinetics in man: normolipemic subjects, familial hypertriglyceridemia and familial combined hyperlipidemia. Metabolism 30: 856-868

[9] Venkatesan S, Cullen P, Pacy P, Halliday D, Scott J (1993) Stable isotopes show a direct relation between VLDL apoB overproduction and serum triglyceride levels and indicate a metabolically and biochemically coherent basis for familial combined hyperlipidemia. Arterioscler Thromb 13: 1110-1118

[10] Cabezas MC, de Bruin TW, Jansen H, Kock LA, Kortlandt W, Erkelens DW (1993) Impaired chylomicron remnant clearance in familial combined hyperlipidemia. Arterioscler Thromb 13: 804-814

[11] Malmstrom R, Packard CJ, Caslake M, et al. (1997) Defective regulation of triglyceride metabolism by insulin in the liver in NIDDM. Diabetologia 40: 454-462

[12] Adiels M, Taskinen MR, Packard C, et al. (2006) Overproduction of large VLDL particles is driven by increased liver fat content in man. Diabetologia 49: 755-765

[13] Veerkamp MJ, de Graaf J, Stalenhoef AF (2005) Role of insulin resistance in familial combined hyperlipidemia. Arterioscler Thromb Vasc Biol 25: 1026-1031 
[14] de Bruin TW, Georgieva AM, Brouwers MC, Heitink MV, van der Kallen CJ, van Greevenbroek MM (2004) Radiological evidence of nonalcoholic fatty liver disease in familial combined hyperlipidemia. Am J Med 116: 847-849

[15] Brouwers MC, Bilderbeek-Beckers MA, Georgieva AM, van der Kallen CJ, van Greevenbroek MM, de Bruin TW (2007) Fatty liver is an integral feature of familial combined hyperlipidaemia: relationship with fat distribution and plasma lipids. Clin Sci (Lond) 112: 123-130

[16] Donnelly KL, Smith CI, Schwarzenberg SJ, Jessurun J, Boldt MD, Parks EJ (2005) Sources of fatty acids stored in liver and secreted via lipoproteins in patients with nonalcoholic fatty liver disease. J Clin Invest 115: 1343-1351

[17] Westerbacka J, Corner A, Tiikkainen M, et al. (2004) Women and men have similar amounts of liver and intra-abdominal fat, despite more subcutaneous fat in women: implications for sex differences in markers of cardiovascular risk. Diabetologia 47: 1360-1369

[18] Brouwers MC, Cantor RM, Kono N, et al. (2006) Heritability and genetic loci of fatty liver in familial combined hyperlipidemia. J Lipid Res 47: 2799-2807

[19] Schindhelm RK, Dekker JM, Nijpels G, et al. (2006) Alanine aminotransferase predicts coronary heart disease events: A 10-year follow-up of the Hoorn Study. Atherosclerosis

[20] Sattar N, Scherbakova O, Ford I, et al. (2004) Elevated alanine aminotransferase predicts new-onset type 2 diabetes independently of classical risk factors, metabolic syndrome, and $\mathrm{C}$-reactive protein in the west of Scotland coronary prevention study. Diabetes 53: $2855-2860$

[21] Suzuki A, Angulo P, Lymp J, et al. (2005) Chronological development of elevated aminotransferases in a nonalcoholic population. Hepatology 41: 64-71

[22] Brunt EM, Janney CG, Di Bisceglie AM, Neuschwander-Tetri BA, Bacon BR (1999) Nonalcoholic steatohepatitis: a proposal for grading and staging the histological lesions. Am J Gastroenterol 94: 2467-2474

[23] Voors-Pette C, de Bruin TW (2001) Excess coronary heart disease in Familial Combined Hyperlipidemia, in relation to genetic factors and central obesity. Atherosclerosis 157: 481-489

[24] Farrell GC (2002) Drugs and steatohepatitis. Semin Liver Dis 22: 185-194

[25] Matthews DR, Hosker JP, Rudenski AS, Naylor BA, Treacher DF, Turner RC (1985) Homeostasis model assessment: insulin resistance and beta-cell function from fasting plasma glucose and insulin concentrations in man. Diabetologia 28: 412-419

[26] van der Kallen CJ, Voors-Pette C, de Bruin TW (2004) Abdominal obesity and expression of familial combined hyperlipidemia. Obes Res 12: 2054-2061

[27] Veerkamp MJ, de Graaf J, Bredie SJ, Hendriks JC, Demacker PN, Stalenhoef AF (2002) Diagnosis of familial combined hyperlipidemia based on lipid phenotype expression in 32 families: results of a 5-year follow-up study. Arterioscler Thromb Vasc Biol 22: 274-282

[28] McNeely MJ, Edwards KL, Marcovina SM, Brunzell JD, Motulsky AG, Austin MA (2001) Lipoprotein and apolipoprotein abnormalities in familial combined hyperlipidemia: a 20 
year prospective study. Atherosclerosis 159: 471-481

[29] Delawi D, Meijssen S, Castro Cabezas M (2003) Intra-individual variations of fasting plasma lipids, apolipoproteins and postprandial lipemia in familial combined hyperlipidemia compared to controls. Clin Chim Acta 328: 139-145

[30] Westerbacka J, Lammi K, Hakkinen AM, et al. (2005) Dietary fat content modifies liver fat in overweight nondiabetic subjects. J Clin Endocrinol Metab 90: 2804-2809

[31] Nielsen S, Guo Z, Johnson CM, Hensrud DD, Jensen MD (2004) Splanchnic lipolysis in human obesity. J Clin Invest 113: 1582-1588

[32] Vozarova B, Stefan N, Lindsay RS, et al. (2002) High alanine aminotransferase is associated with decreased hepatic insulin sensitivity and predicts the development of type 2 diabetes. Diabetes 51: 1889-1895

[33] ter Avest E, Sniderman AD, Bredie SJ, Wiegman A, Stalenhoef AF, de Graaf J (2007) Effect of aging and obesity on the expression of dyslipidaemia in children from families with familial combined hyperlipidaemia. Clin Sci (Lond) 112: 131-139

[34] Karjalainen L, Pihlajamaki J, Karhapaa P, Laakso M (1998) Impaired insulin-stimulated glucose oxidation and free fatty acid suppression in patients with familial combined hyperlipidemia: a precursor defect for dyslipidemia? Arterioscler Thromb Vasc Biol 18: $1548-1553$ 
Genetic predisposition to develop fatty liver

Heritability and genetic loci of fatty liver in familial combined hyperlipidemia

Brouwers MC, Cantor RM, Kono N Yoon JL, van der Kallen CJ Bilderbeek-Beckers MA van Greevenbroek MM Lusis AJ, de Bruin TW

J Lipid Res. 2006; 47(12):2799-807 


\section{Abstract}

VLDL-overproduction, a process that is driven by an excess amount of hepatic fat, is a welldocumented feature of familial combined hyperlipidemia (FCHL). The aim of the present study was to investigate whether fatty liver, measured with ultrasound and as plasma alanine aminotransferase (ALT) levels, develops against a genetic background in FCHL, and to identify chromosomal loci that are linked to these traits.

In total, 157 FCHL family members and 20 spouses participated in the present study. Radiological evidence of fatty liver was more prevalent not only in FCHL probands (40\%), but also in their relatives $(35 \%)$ compared to spouses $(15 \%)(p<0.05)$. Heritability calculations revealed that $20-36 \%$ of the variability in ALT levels could be attributed to genetic factors. Non-parametric quantitative trait locus (QTL) analysis revealed three significant $(\mathrm{p}<0.001)$ loci with either the ultrasound or ALT trait in the male sample: 1q42.3, 7p12-21 and 22p13-q11, but none in the female sample or the entire group. Of these QTL, the 7p region was consistent over time, since re-analysis with ALT levels that were determined during a five-years earlier visit yielded similar results.

The present study shows that fatty liver is a heritable aspect of FCHL. Replication of particularly the $7 \mathrm{p}$ region is awaited. 


\section{Introduction}

More than three decades ago, familial combined hyperlipidemia (FCHL) was delineated as a highly prevalent $(1: 100)$, primary hyperlipidemia that was associated with premature coronary artery disease [1]. Although it was initially assumed that FCHL was inherited as an autosomal dominant disease [1], subsequent studies have revealed that FCHL follows a complex segregation pattern $[2,3]$. However, to date a true understanding of the genetic background of FCHL is still lacking, despite numerous genome screens and positional candidate association studies with FCHL-related traits, such as plasma triglycerides, total cholesterol, apolipoprotein B and LDL particle size [4-7]. One major breakthrough in the genetic dissection of FCHL was the recent identification and subsequent confirmation of upstream stimulatory factor 1 (USF1) located on chromosome 1q21-23 [8, 9]. Functional evidence is now accumulating that variants in the USF1gene can indeed contribute to the development of hyperlipidemia [10,11].

Nowadays, it is commonly accepted that the elevated plasma triglycerides, total cholesterol and apolipoprotein B in FCHL are heterogeneous in origin, since they are the consequence of both an overproduction of VLDL-particles as well as a decreased clearance of remnants [12-14]. Researchers have therefore stated that lipid levels are not the optimal phenotypical markers for elucidating the genetic background of FCHL [15]. However, easily obtainable markers that directly reflect either overproduction or clearance of lipid particles have not been available.

Previous experimental studies in human subjects have suggested that the production of VLDLparticles is driven by the amount of fat that is supplied to the liver [16, 17]. Adiels and colleagues confirmed this assumption by reporting that, in both normal subjects and patients with type 2 diabetes mellitus, the production of VLDL-particles is indeed related with the hepatic fat content [18]. Of interest, our laboratory recently demonstrated that an increased hepatic fat content is a common feature of FCHL as well, in particular in those patients with the hypertriglyceridemic phenotype [19].

Therefore, the present study was conducted to investigate whether an increased hepatic fat content is involved in the genetic background of FCHL. For this purpose, surrogates of fatty liver, i.e. liver ultrasound and plasma alanine aminotransferase (ALT) levels, were determined in our welldefined FCHL pedigrees and correlated with the traditional FCHL lipid traits. Subsequently, the heritability of fatty liver was estimated and a quantitative trait linkage (QTL) analysis was performed to search the genome for chromosomal loci implicated in the pathogenesis of fatty liver and hyperlipidemia in FCHL.

\section{Materials and methods}

\section{Study population}

FCHL patients, their relatives and spouses were ascertained as previously described [20]. Since FCHL family members and their spouses share a similar environment, any observed statistical difference is likely to be explained by genetic factors. It should therefore be noted that the spouses do not necessarily represent a random sample from the general population.

All subjects visited our lipid clinic after an overnight fast and total abstinence from alcohol for 
the last three days. They were withdrawn from lipid medication two weeks prior to their visit. Furthermore, subjects did not consume more than two units of alcohol daily, did not report recent rapid weight loss and did not use any medication associated with the development of fatty liver [21]. One subject was excluded from the present study because of positive serology for hepatitis C.

This study was approved by the Human Investigation Research Committee of the Academic Hospital of Maastricht/Maastricht University and the UCLA institutional review board. All subjects gave written informed consent.

Height was determined with a stadiometer, and weight was measured while wearing only underwear. Body mass index (BMI) was calculated as weight divided by height squared $(\mathrm{kg} /$ $\left.\mathrm{m}^{2}\right)$.

\section{Plasma measurements}

Blood was collected in pre-cooled EDTA tubes. After centrifugation at $3000 \mathrm{rpm}$ for $15 \mathrm{~min}$ at $4^{\circ} \mathrm{C}$, plasma aliquots were stored at $-80^{\circ} \mathrm{C}$. Apolipoprotein $\mathrm{B}$, total cholesterol, HDL-cholesterol and triglycerides were measured as described previously [20]. Alanine aminotransferase (ALT) levels were measured with a commercially available assay (Ecoline ${ }^{\circledR} \mathrm{S}+$, DiaSys Diagnostic Systems GmbH, Germany). ALT levels were also determined in plasma samples that were withdrawn during all subjects' screening visits, five years earlier $\left(\mathrm{ALT}_{1999}\right)$. Preparation of subjects and handling of plasma in 1999 were similar as described above.

\section{Ultrasound}

Ultrasound of the liver was performed with an ATL9 HDI (Bothel, USA) ultrasound system, using a C7-4 and a C4-2 transducer. Standardized images and movies of the liver and right kidney were recorded on videotape and examined by a radiologist unaware of the subject's clinical characteristics. Criteria for fatty liver were as previously described [22, 23]. In short, the four classifications were: 1) normal liver, normal hepatic echotexture and normal beam attenuation; 2) mild steatosis, slight increase in echogenicity of liver parenchyma in comparison with right kidney, with minimal or no decrease of visualization of hepatic vessels and diaphragm; 3) moderate steatosis, diffuse increase in echogenicity of the liver with slightly impaired visualization of intrahepatic vessels and diaphragm; 4) severe steatosis, marked increase of liver echogenicity, poor visualization of intrahepatic vessels and increased posterior beam attenuation represented by nonvisualization of the diaphragm. The intra-observer agreement expressed as kappa, determined in 30 random scans, was good $(x=0.68)$ and in agreement with earlier studies [23].

\section{Genotyping}

Genotyping of the 377 informative microsatellite markers was done by the Marshfield Genotyping service, as described previously [7]. Markers of Marshfield panel 10 were used with an average intermarker distance of 9.4cM. In total, 157 FCHL family members and 20 spouses were genotyped. 
Statistical and genetic analyses

Population characteristics:

Differences in general descriptives between FCHL probands, their relatives and spouses were calculated with linear regression for continuous traits and with logistic regression for binary traits, both with inclusion of age and sex. FCHL status was entered as a dummy variable into these models $($ FCHL proband/relative $=1$, spouse $=0)$. Sex specific correlations between fatty liver measured with ultrasound and ALT levels were calculated with ordinal regression. All the above analyses were conducted with SPSS 13.0 statistical package (SPSS Inc, Illinois USA).

Heritability of ALT levels:

Familiality of ALT levels, the continuous trait used as a surrogate for fatty liver, was assessed in two ways. First, the FCOR subprogram of the SAGE software package [24] was used to estimate the intraclass correlation for sibling pairs. This correlation is of relevance, since our linkage analyses were also conducted in sibling pairs (see below). Intraclass correlations were also calculated for plasma triglycerides, total cholesterol, HDL-cholesterol and apolipoprotein B levels.

Additionally, the variance component model implemented in SOLAR [25] was used to estimate familiality of the normalized deviates of ALT. Similar analyses were performed for ALT values adjusted for BMI.

It should be noted that the heritability estimate is strongly dependent on both the reproducibility of the essay, which was high for ALT (coefficient of variation: 3.5\%), and the temporal (biological) variation in the trait value.

Identification of genetic loci:

Multipoint QTL analysis at $1 \mathrm{cM}$ intervals was conducted using the nonparametric modelfree analysis option of the Genehunter software [26] that correlates the differences in trait levels within sibling pairs with their degree of allele sharing identitical by descent along the chromosomes. The linkage map was sex-averaged. In case of linkage in a region, similar trait values, i.e. plasma ALT levels or liver ultrasound stages, are expected to occur in sibling pairs with increased marker allele sharing, whereas those pairs that have markedly different trait values will exhibit less marker allele sharing. This is assessed with a statistical test similar to the KruskalWallis test. A p-value $<0.001$ was regarded as significant to identify a QTL. For continuous traits with more normal distributions, i.e. ALT levels, the Haseman-Elston option of the Genehunter software was used as well. It correlates allele-sharing identical by descent with the squares of trait differences in sibling pairs by regressing the squared trait differences against the estimated allele sharing. 


\section{Results}

\section{Sample characteristics}

Descriptive statistics of FCHL probands, their relatives and spouses are displayed in Table 1. FCHL probands had higher plasma triglycerides and total cholesterol levels in comparison with their spouses, and apo B levels were increased, but not significantly. Furthermore, both surrogates of fatty liver, i.e. plasma ALT and liver ultrasound, suggest a higher prevalence of fatty liver, not only in the FCHL probands, but also in their relatives (Table 1).

Of note, in all FCHL family members (probands and relatives combined), plasma ALT levels were related with the different stages of fatty liver as measured with ultrasound. $(r=0.49, \mathrm{p}<$ 0.001; ordinal regression). Furthermore, ALT levels were within the normal range. However, as shown in Figure 1, a marked sex difference was observed for this ALT-ultrasound relation $(\mathrm{r}=$ $0.69, \mathrm{p}<0.001$ in men versus $\mathrm{r}=0.27, \mathrm{p}=0.03$ for women), a phenomenon that has also been found for the relation ALT-fatty liver as measured with magnetic resonance spectroscopy [27].

Table 1.

Characteristics of FCHL patiens, NL relatives and spouses

\begin{tabular}{lccc}
\hline & Spouses & Relatives & FCHL probands \\
\hline Male / female & $9 / 11$ & $67 / 70$ & $12 / 8$ \\
Age, years & $58.6 \pm 9.2$ & $45.4 \pm 14.9^{*}$ & $57.1 \pm 9.1$ \\
$\mathrm{BMI}, \mathrm{kg} / \mathrm{m} 2$ & $26.1(22.9-28.7)$ & $25.8(23.1-28.9)$ & $27.0(24.3-28.6)$ \\
Apolipoprotein B, g/L & $1.0 \pm 0.3$ & $1.1 \pm 0.3 \dagger$ & $1.2 \pm 0.3$ \\
Cholesterol, mmol/L & $5.6 \pm 1.0$ & $5.6 \pm 1.6$ & $6.9 \pm 2.2 \dagger$ \\
HDL-cholesterol, mmol/L & $1.1 \pm 0.3$ & $0.9 \pm 0.3$ & $0.9 \pm 0.3$ \\
Triglycerides, mmol/L & $1.1(1.0-1.9)$ & $1.3(1.0-2.0) \dagger$ & $3.1(1.4-3.8) \dagger$ \\
ALT, U/L & $15.2(13.1-17.8)$ & $18.2(13.8-25.6) \dagger$ & $21.7(16.6-26.1) \dagger$ \\
Fatty liver (US) (\%) & 15 & $35 \ddagger$ & $40 \ddagger$ \\
\hline
\end{tabular}

Data are presented as mean \pm SD or as median (interquartile range). Abbreviations: BMI: body mass index, ALT: alanine aminotransferase, US: ultrasound.

${ }^{*} \mathrm{p}<0.05$, in comparison with spouses, student's T-test.

$t_{\mathrm{p}}<0.05$, in comparison with spouses, linear regression, age and gender adjusted

$\ddagger \mathrm{p}<0.05$, in comparison with spouses, logistic regression, age and gender adjusted

All analyses were Hochberg corrected 


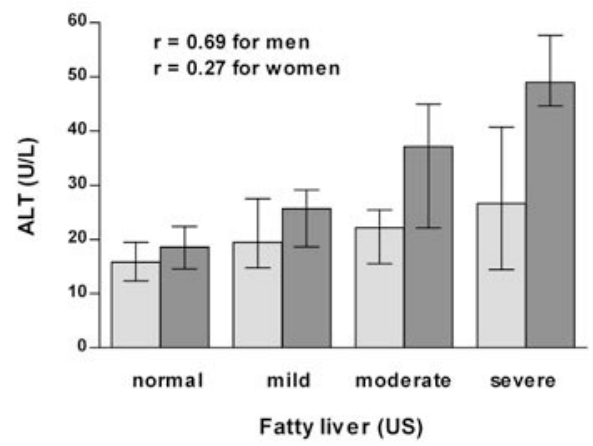

Figure 1.

Relation of fatty liver severity by ultrasound (US) versus plasma ALT levels in FCHL family members $(\mathrm{n}=157)$, split by sex (dark bars, men; light bars, women). ALT levels are presented as medians with interquartile range. Correlation coefficients were calculated with ordinal regression (Nagelkerke pseudo r)

\section{Relation of surrogates of fatty liver with plasma lipid levels}

To assess if fatty liver is related to the plasma lipid levels that are used to determine the characteristic FCHL phenotype, we calculated correlations of the surrogates of fatty liver with plasma apolipoprotein B, total cholesterol and triglycerides in all FCHL family members, i.e. probands and relatives combined. As shown in Figure 2, no significant correlations were observed for apolipoprotein B and total cholesterol levels with the ultrasound assessment of fatty liver (panels A and C), and marginal associations were found with ALT levels (panels B and D). A much stronger correlation was observed between the surrogates of fatty liver and plasma triglyceride concentrations (panel $\mathrm{E}$ and F).

\section{Heritability of ALT levels}

The intraclass correlation estimated with SAGE FCOR [24] in 230 sibling pairs (Table 2) was 0.18 for $\log$ ALT (sex corrected), corresponding with a maximum heritability estimate of 0.36 $\left(2 \mathrm{r}=\mathrm{h}^{2}\right)$ [28]. Intraclass correlations were $0.12\left(\mathrm{~h}^{2}=0.24\right)$ for plasma triglycerides, $0.27\left(\mathrm{~h}^{2}=0.54\right)$ for total cholesterol, $0.23\left(h^{2}=0.46\right)$ for HDL-cholesterol and $0.08\left(h^{2}=0.16\right)$ for apolipoprotein $\mathrm{B}$ levels, indicating that the maximum heritability for the traditional FCHL traits are in a similar range as ALT levels.

Heritability estimates for ALT levels when all family relationships were included were a maximum of 0.20 ( $p=0.09$, SOLAR). Of interest, a correction for BMI by including it as a covariate in the model did not reduce the value of the maximum heritability estimates for $\log \operatorname{ALT}(0.27, \mathrm{p}=$ 0.03 , SOLAR), implying that BMI does not appear to be a major contributor to these heritability estimates. 

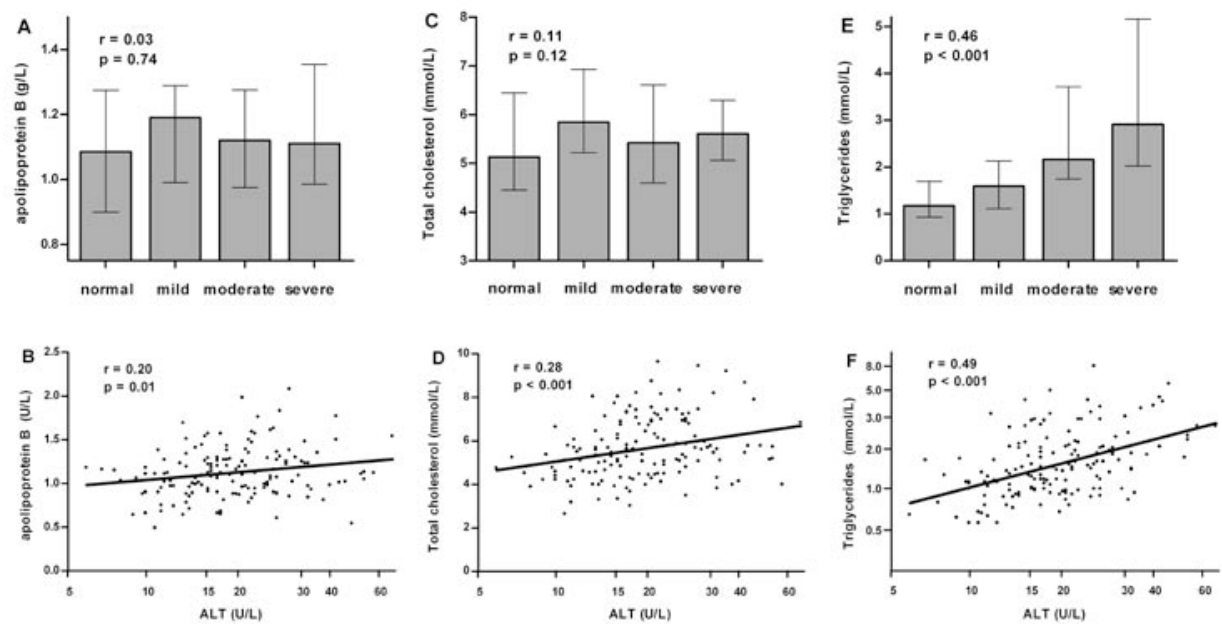

Figure 2.

Relation of ultrasound stages of fatty liver and ALT levels with apolipoprotein B (A, B), total cholesterol (C, D), and triglycerides (E, F) in FCHL family members. Data in A, C and E are presented as medians with interquartile ranges. ALT levels are presented on a $\log$ scale

Table 2 .

Sibships in heritability and QTL analyses

\begin{tabular}{lccccccccc}
\hline Sibship size & 2 & 3 & 4 & 5 & 6 & 7 & 9 & 11 & Total \\
\hline N of sibships & $28(18)$ & $12(2)$ & $5(2)$ & 3 & 2 & 1 & $(1)$ & 1 & $52(23)$ \\
N of individuals & $56(34)$ & $36(6)$ & $20(8)$ & 15 & 12 & 7 & $(9)$ & 11 & $157(57)$ \\
N of sibpairs & $28(18)$ & $36(6)$ & $30(12)$ & 30 & 30 & 21 & $(36)$ & 55 & $230(72)$ \\
N of independent & $28(18)$ & $24(4)$ & $15(6)$ & 12 & 10 & 6 & $(8)$ & 10 & $105(36)$ \\
sibpairs & & & & & & & & & \\
\hline
\end{tabular}

Data are presented for the total FCHL population (men and women combined). The data for the male sample are presented in parentheses 
QTL analysis for surrogates of fatty liver

Non-parametric multipoint QTL analyses were conducted for log ALT (sex corrected; continuous trait) and for the stages of fatty liver (US) (ordinal trait). This analysis did not reveal any QTL when the stringent criterion of $\mathrm{p}<0.001$ was applied. Since a sex specific relation was found for plasma ALT levels with fatty liver stages (US) (Figure 1), QTL analyses were subsequently conducted by splitting the sample by sex. Although the male sample was reduced to 72 sibpairs (Table 2$)$, one borderline significant $(p=0.001)$ and two significant $(p<0.001)$ QTL were observed for either log ALT levels or fatty liver (US). These were located on chromosome 1q42.3 $(\mathrm{p}=0.001$, for fatty liver [US]), 7p12-21 ( $\mathrm{p}=0.0002$, for log ALT) and 22p13-q11 ( $\mathrm{p}=0.0007$, for fatty liver [US]), see also Table 3. No QTL were found in the female sample. The male sample contained one potentially influential large pedigree consisting of nine siblings (Table 2), however similar QTL results were obtained when the analysis was conducted without this particular family (data not shown). The peak marker, the marker distance (from p-terminal end of the chromosome) and the Haseman-Elston LOD-score for the three QTL are also presented in Table 3. Plots of the QTL for log ALT and fatty liver (US) in the male FCHL sample are displayed in Figure 3. Of interest, the QTL plots for fatty liver (US) and log ALT are very similar, which likely reflects the correlation between these traits in the male population $(r=0.69)$, as was shown in Figure 1. Furthermore, the QTL plots for $\log$ ALT and $\log$ ALT corrected for BMI are similar in shape in all three graphs, illustrating that BMI does not contribute to the evidence for linkage to this region (Figure 3). Of interest, linkage peaks were observed for BMI only at 1q42.3 and 7 p12-21, but not at 22p13-q11 (Figure 3).

Table 3.

QTL for fatty liver (US) and log ALT by $\mathrm{p}<0.001$ in multipoint analyses of male FCHL sibships

\begin{tabular}{lccccc}
\hline Quantitative trait & $\begin{array}{c}\text { Marker closest } \\
\text { to QTL }\end{array}$ & $\begin{array}{c}\text { QTL chromosome } \\
\text { band }\end{array}$ & $\begin{array}{c}\text { Marker distance } \\
\text { from telomere }\end{array}$ & $\begin{array}{c}\text { Non-parametic } \\
\text { Z-score (p) }\end{array}$ & $\begin{array}{c}\text { HE } \\
\text { Lod }\end{array}$ \\
\hline Fatty liver (US) & D1S235 & $1 \mathrm{q} 42.3$ & 251 & $3.1(0.001)$ & \\
Log ALT & D1S3462 & $1 \mathrm{q} 42.2$ & 243 & $2.7(0.004)$ & 1.8 \\
& & & & & \\
Fatty liver (US) & D7S2846 & $7 \mathrm{p} 14.1$ & 56 & $2.2(0.01)$ & \\
Log ALT & D7S2846 & $7 \mathrm{p} 14.1$ & 62 & $3.6(0.0002)$ & 2.8 \\
& & & & & \\
Fatty liver (US) & D22S686 & 22q11.22 & 12 & $3.2(0.0007)$ & \\
Log ALT & D22S686 & 22q11.22 & 9 & $2.4(0.008)$ & 1.3 \\
\hline
\end{tabular}

Abbreviations: US: ultrasound, QTL: quantitative trait locus, HE: Haseman-Elston, ALT: alanine aminotransferase. HE Lod-score is only given for the continuous trait log ALT (see material and methods) 

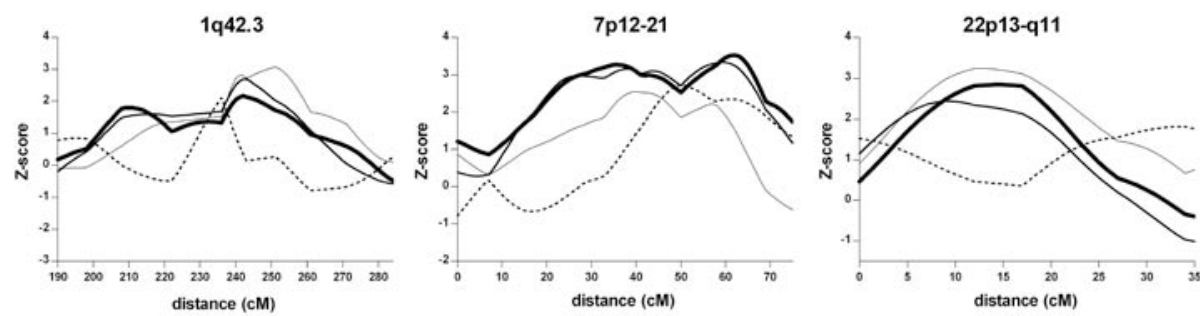

Figure 3.

QTL identified by $\mathrm{p}<0.001$ for either fatty liver (ultrasound, US; thin line) or log ALT (intermediate line) in male FCHL sibships. QTL are also shown for log ALT adjusted for BMI (bold line) and for BMI only (dashed line).

\section{QTL analysis for plasma triglycerides levels in $1 q$, $7 p$ and $22 q$ region}

Since both surrogates of fatty liver were correlated with plasma triglycerides (Figure 2, panel $\mathrm{E}$ and $\mathrm{F}$ ), one of the diagnostic hallmarks of FCHL, we subsequently decided to conduct QTL analyses for plasma triglycerides in the three significant regions. As shown in Figure 4, the Z-scores for plasma triglycerides were markedly lower than the original QTL for log ALT. However, the plots for triglycerides were similarly shaped, in particular the $7 \mathrm{p}$ region (Figure 4, panel B), which probably reflects the plasma triglycerides-ALT relation. This relation was also reflected by a diminished linkage signal of the original QTL at 7p and 22q, when ALT levels were corrected for plasma triglycerides (Figure 4, panel B and C). The unchanged QTL at 1q (Figure 4, panel A) probably indicates that this locus is specific for ALT levels, but does not affect plasma triglycerides.

\section{QTL analysis for $A L T_{1999}$ levels in $1 q, 7 p$ and $22 q$ region}

To further substantiate the observed linkage peaks, QTL analyses were conducted for log ALT levels that were determined in all subjects during their screenings visit, five years prior to the current study (further referred to as $\mathrm{ALT}_{1999}$ ). In addition, $\mathrm{ALT}_{1999}$ levels were also corrected for BMI, which was determined five years earlier $\left(\mathrm{BMI}_{1999}\right)$. Similar to our previous report [29] we assumed that, since a genetic predisposition does not change over time, QTL are not expected to change over time either, unless there is a strong environmental component that affects the trait [29]. Of note, a very similar intraclass correlation and heritability estimate were observed for $\mathrm{ALT}_{1999}$ levels in the overall FCHL population (intraclass correlation: 0.18 [SAGE], maximum heritability: 0.19 [SOLAR]).

As shown in Figure 5, panel A and C, the QTL for the original ALT (2004) trait did not appear at the $1 \mathrm{q}$ and $22 \mathrm{q}$ region when analyzed with the $\mathrm{ALT}_{1999}$ values, nor with $\mathrm{ALT}_{1999}$ adjusted for $\mathrm{BMI}_{1999}$. In contrast, the QTL on chromosome 7 was observed with the $\mathrm{ALT}_{1999}$ and $\mathrm{ALT}_{1999}$ adjusted for $\mathrm{BMI}_{1999}$ traits (Figure 5, panel B). Furthermore, the QTL was narrowed from 7p1221 to $7 \mathrm{p} 12-15\left(\mathrm{Z}_{\max }=2.7\right.$ at $33 \mathrm{cM}, \mathrm{p}=0.003$ for $\mathrm{ALT}_{1999}$ adjusted for $\left.\mathrm{BMI}_{1999}\right)$. We interpret this 
to mean that, when the linkage results for $\mathrm{ALT}_{2004}$ and $\mathrm{ALT}_{1999}$ levels are compared, the observed QTL on chromosome 7 is least likely to result from a Type 1 statistical error.

Of note, the linkage results for $\mathrm{BMI}_{1999}$ were very similar to the original results for BMI (data not shown).
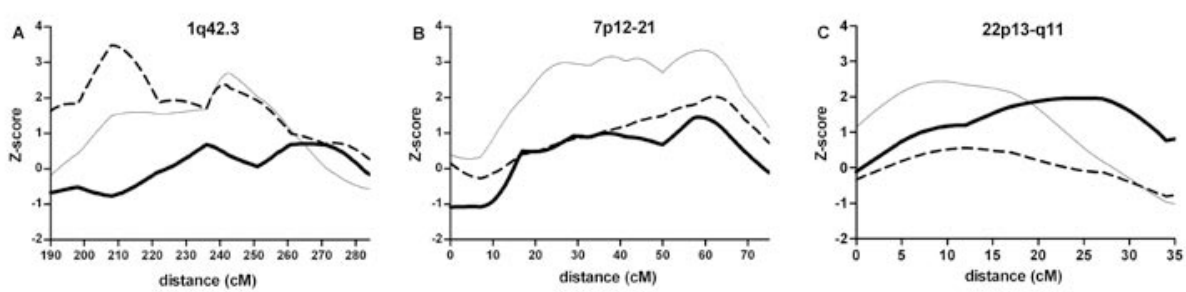

Figure 4.

QTL for plasma triglycerides (bold solid line) and for ALT levels corrected for triglycerides (bold dashed line) compared to the original QTL for $\log$ ALT (thin solid line) in 1q, 7p and 22q region
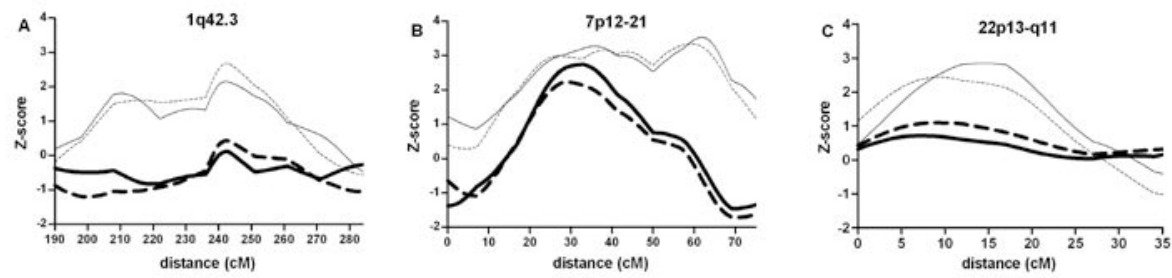

Figure 5.

QTL for $\log \mathrm{ALT}_{1999}$ (bold dashed line) and $\mathrm{ALT}_{1999}$ adjusted for BMI (bold solid line) compared to the original QTL (2004) for $\log$ ALT (thin dashed line) and $\log$ ALT adjusted for BMI (thin solid line) in 1q, 7p and 22q region 


\section{Discussion}

The current study was conducted to explore whether fatty liver develops against a genetic background in FCHL and, when affirmative, whether the fatty liver trait can be used in linkage analysis to unravel the genetic susceptibility to develop FCHL. These research questions were triggered by our previous observation of an increased prevalence of fatty liver in an outpatient FCHL population [19] and the recently reported relation between fatty liver and VLDLoverproduction in normal subjects and patients with type 2 diabetes mellitus [18].

Both surrogates of fatty liver that were used in the present study, i.e. liver ultrasound and plasma alanine aminotransferase (ALT) levels, were significantly correlated with plasma triglycerides levels in all FCHL family members, analogous to what has been observed in non-FCHL populations [18]. This relation probably reflects the relation between fatty liver and the overproduction of VLDL-particles, a well-documented feature of FCHL $[12,13]$. Of interest, we very recently reported that VLDL-apo B levels are also related with the amount of hepatic fat in FCHL [30]. Although this observation seems to be in contrast with the current almost absent relation between fatty liver surrogates and total apo B levels, it is most likely explained by the fact that only a minor part $(<10 \%)$ of the variation in total apo B levels is accounted for by VLDL-apo B [31].

The current study demonstrated that the prevalence of fatty liver as measured with ultrasound was increased not only in FCHL probands, but also in their relatives, which is suggestive of the presence of a genetic component. Similar results were obtained with ALT levels, a liver specific enzyme that was, within its normal range, associated with the ultrasound stages of fatty liver. The suggestion of a genetic component was subsequently confirmed by using two different analytical strategies as implemented in SOLAR [25] and SAGE [24]: 20-36\% of the variability in fatty liver, measured as plasma ALT levels, can be attributed to genetic factors. This is the first study that has estimated the contribution of genetic factors to variations in fatty liver. This contribution was within the same range as the more traditional FCHL traits that were included as a reference. Of note, the high maximum heritability for total cholesterol and HDL-cholesterol and the low heritability for plasma triglycerides are in agreement with previous reports [32, 33].

A subsequent genomic search for chromosomal regions associated with surrogate markers of fatty liver, i.e. ultrasound and ALT levels, revealed three loci in the male sample that fulfilled our significance criterion: 1q42.3, 7p12-21 and 22p13-q11 (Genehunter software [26]), whereas no QTL were observed in the female sample and overall population. We are reporting QTL with $\mathrm{p}<0.001$, but recognize that appropriate correction for multiple-testing has not been made. Such a correction is not straightforward, since two of the samples (male and female) are nested in the third (overall population). We are reporting these results, and suggest replication prior to additional gene finding efforts in these regions.

The three observed QTL in the male sample were not confounded by obesity, an important causal factor in the development of fatty liver [34, 35], since correction for body mass index 
did not affect the original QTL. Analysis with only BMI resulted in a linkage peaks at 1q and $7 \mathrm{p}$ as well. The currently observed QTL have not been reported before in FCHL. Although linkage has been noted for apolipoprotein AII levels in the 1q41 region [36], it is probably too far away from our observed QTL for fatty liver at 1q42.3. Since we are the first to use this refined phenotype, it is possible that we have detected QTL that would not have been found with the traditional FCHL traits. This is illustrated by our linkage results for plasma triglycerides: although the curve was similarly shaped for triglycerides and ALT levels (Figure 4), the results for triglycerides did by far not reach significance. Of note, a QTL for BMI has been reported before for the $7 \mathrm{p}$ region [37-39].

The sex-specificity of our findings is not exceptional, since very recently it was shown that gender-specific genetic effects are observed for many common traits, such as HDL-cholesterol, blood pressure, eosinophilia and lymphocyte count [40]. Of note, most significant linkage results were found in the male population [40], probably due to the more homogeneous nature of that sample. Since estrogens exert well-known effects on lipid and liver metabolism [41-43], variations in estrogen concentrations among premenopausal and postmenopausal women have probably introduced a substantial noise in our dataset.

The aspect of false-positive results is a frequently encountered problem in the field of linkage analysis [44]. Experts have therefore proposed guidelines to reduce the occurrence of type 1 errors as much as possible [44, 45]. We applied, similar to our previous report [29], an alternate strategy that can be regarded as a first validation step to avoid false-positive results [46]: since the maximum heritability of ALT levels did not change over time, we assumed that a true QTL should not change over time either. QTL analysis was therefore repeated with ALT levels that were determined during another visit, five years earlier. Re-analysis revealed that, of the three initially observed QTL, the one at 7p is most worthwhile to pursue, whereas the 1q and 22q regions may be type 1 errors.

Power is an additional important criterion for QTL analyses. Given the number of sibling pairs in our sample, we have $80 \%$ power to identify QTL with locus specific heritabilities of $15 \%$ or greater when a 0.05 level of significance is set. Given our stringent criterion of 0.001 , we have sufficient power to identify QTL of 30\% locus specific heritabilities, and thus are likely to have missed many QTL of smaller effects.

In the present study, ultrasound and ALT levels were used as surrogates of fatty liver. The gold standard, i.e. liver biopsy, was ethically not acceptable in this relatively healthy population. Furthermore, the use of magnetic resonance spectroscopy (MRS), an alternative method to assess the amount of hepatic fat, is limited in large-sized populations, such as the present one. Nevertheless, we are confident that we have carefully studied the role of genetic loci in fatty liver in men, since both ultrasound, a validated measure of moderate and severe stages of fatty liver [23], and ALT, a plasma marker that is, within its normal range, fairly correlated with hepatic fat on MRS [27], yielded similar QTL outcomes in the male population. 
In summary, the present study has demonstrated that fatty liver is a heritable aspect of FCHL. A subsequent genomic screen for surrogates of fatty liver revealed three QTL in the male sample, of which the chromosomal 7p12-15 region appeared to be the most consistent QTL over time. Replication and fine mapping of this region are therefore awaited.

\section{References}

[1] Goldstein JL, Schrott HG, Hazzard WR, Bierman EL, Motulsky AG (1973) Hyperlipidemia in coronary heart disease. II. Genetic analysis of lipid levels in 176 families and delineation of a new inherited disorder, combined hyperlipidemia. J Clin Invest 52: 1544-1568

[2] Jarvik GP, Brunzell JD, Austin MA, Krauss RM, Motulsky AG, Wijsman E (1994) Genetic predictors of FCHL in four large pedigrees. Influence of ApoB level major locus predicted genotype and LDL subclass phenotype. Arterioscler Thromb 14: 1687-1694

[3] Pajukanta P, Terwilliger JD, Perola M, et al. (1999) Genomewide scan for familial combined hyperlipidemia genes in finnish families, suggesting multiple susceptibility loci influencing triglyceride, cholesterol, and apolipoprotein B levels. Am J Hum Genet 64: $1453-1463$

[4] Allayee H, Krass KL, Pajukanta P, et al. (2002) Locus for elevated apolipoprotein B levels on chromosome $1 \mathrm{p} 31$ in families with familial combined hyperlipidemia. Circ Res 90: 926-931

[5] Naoumova RP, Bonney SA, Eichenbaum-Voline S, et al. (2003) Confirmed Locus on Chromosome 11p and Candidate Loci on $6 \mathrm{q}$ and $8 \mathrm{p}$ for the Triglyceride and Cholesterol Traits of Combined Hyperlipidemia. Arterioscler Thromb Vasc Biol

[6] Mar R, Pajukanta P, Allayee H, et al. (2004) Association of the APOLIPOPROTEIN A1/C3/A4/A5 gene cluster with triglyceride levels and LDL particle size in familial combined hyperlipidemia. Circ Res 94: 993-999

[7] Cantor RM, de Bruin T, Kono N, et al. (2004) Quantitative trait loci for apolipoprotein $\mathrm{B}$, cholesterol, and triglycerides in familial combined hyperlipidemia pedigrees. Arterioscler Thromb Vasc Biol 24: 1935-1941

[8] Pajukanta P, Lilja HE, Sinsheimer JS, et al. (2004) Familial combined hyperlipidemia is associated with upstream transcription factor 1 (USF1). Nat Genet 36: 371-376

[9] Huertas-Vazquez A, Aguilar-Salinas C, Lusis AJ, et al. (2005) Familial combined hyperlipidemia in Mexicans: association with upstream transcription factor 1 and linkage on chromosome 16q24.1. Arterioscler Thromb Vasc Biol 25: 1985-1991

[10] Naukkarinen J, Gentile M, Soro-Paavonen A, et al. (2005) USF1 and dyslipidemias: converging evidence for a functional intronic variant. Hum Mol Genet 14: 2595-2605

[11] Hoffstedt J, Ryden M, Wahrenberg H, van Harmelen V, Arner P (2005) Upstream transcription factor-1 gene polymorphism is associated with increased adipocyte lipolysis. J Clin Endocrinol Metab 90: 5356-5360

[12] Venkatesan S, Cullen P, Pacy P, Halliday D, Scott J (1993) Stable isotopes show a direct relation between VLDL apoB overproduction and serum triglyceride levels and indicate 
a metabolically and biochemically coherent basis for familial combined hyperlipidemia. Arterioscler Thromb 13: 1110-1118

[13] Kissebah AH, Alfarsi S, Adams PW (1981) Integrated regulation of very low density lipoprotein triglyceride and apolipoprotein-B kinetics in man: normolipemic subjects, familial hypertriglyceridemia and familial combined hyperlipidemia. Metabolism 30: 856-868

[14] Cabezas MC, de Bruin TW, Jansen H, Kock LA, Kortlandt W, Erkelens DW (1993) Impaired chylomicron remnant clearance in familial combined hyperlipidemia. Arterioscler Thromb 13: 804-814

[15] Porkka KV, Nuotio I, Pajukanta P, et al. (1997) Phenotype expression in familial combined hyperlipidemia. Atherosclerosis 133: 245-253

[16] Malmstrom R, Packard CJ, Caslake M, et al. (1998) Effects of insulin and acipimox on VLDL1 and VLDL2 apolipoprotein B production in normal subjects. Diabetes 47: 779-787

[17] Lewis GF, Uffelman KD, Szeto LW, Weller B, Steiner G (1995) Interaction between free fatty acids and insulin in the acute control of very low density lipoprotein production in humans. J Clin Invest 95: 158-166

[18] Adiels M, Taskinen MR, Packard C, et al. (2006) Overproduction of large VLDL particles is driven by increased liver fat content in man. Diabetologia: 1-11

[19] de Bruin TW, Georgieva AM, Brouwers MC, Heitink MV, van der Kallen CJ, van Greevenbroek MM (2004) Radiological evidence of nonalcoholic fatty liver disease in familial combined hyperlipidemia. Am J Med 116: 847-849

[20] Voors-Pette C, de Bruin TW (2001) Excess coronary heart disease in Familial Combined Hyperlipidemia, in relation to genetic factors and central obesity. Atherosclerosis 157: 481-489

[21] Farrell GC (2002) Drugs and steatohepatitis. Semin Liver Dis 22: 185-194

[22] Fishbein M, Castro F, Cheruku S, et al. (2005) Hepatic MRI for Fat Quantitation: Its Relationship to Fat Morphology, Diagnosis, and Ultrasound. J Clin Gastroenterol 39: 619-625

[23] Saadeh S, Younossi ZM, Remer EM, et al. (2002) The utility of radiological imaging in nonalcoholic fatty liver disease. Gastroenterology 123: 745-750

[24] Sorant AJM, Bonney GE, Elstron RC, Bailey-Wilson JE, Wilson AF (1994) S.A.G.E.: Statistical Analysis for Genetic Epidemiology. Case Western Reserve University, Cleveland, $\mathrm{Oh}$

[25] Almasy L, Blangero J (1998) Multipoint quantitative-trait linkage analysis in general pedigrees. Am J Hum Genet 62: 1198-1211

[26] Kruglyak L, Daly MJ, Reeve-Daly MP, Lander ES (1996) Parametric and nonparametric linkage analysis: a unified multipoint approach. Am J Hum Genet 58: 1347-1363

[27] Westerbacka J, Corner A, Tiikkainen M, et al. (2004) Women and men have similar amounts of liver and intra-abdominal fat, despite more subcutaneous fat in women: implications for sex differences in markers of cardiovascular risk. Diabetologia 47: 1360-1369 
[28] Falconer DS (1967) The inheritance of liability to diseases with variable age of onset, with particular reference to diabetes mellitus. Ann Hum Genet 31: 1-20

[29] Brouwers MC, Kono N, van Greevenbroek MM, et al. (2006) Longitudinal differences in familial combined hyperlipidemia quantitative trait loci. Arterioscler Thromb Vasc Biol 26: e118-119

[30] Brouwers MC, Bilderbeek-Beckers MA, Georgieva AM, van der Kallen CJ, van Greevenbroek MM, de Bruin TW (2007) Fatty liver is an integral feature of familial combined hyperlipidemia: relation with fat distribution and plasma lipids. Clin Sci (Lond) 112: $123-130$

[31] Sniderman A, Vu H, Cianflone K (1991) Effect of moderate hypertriglyceridemia on the relation of plasma total and LDL apo B levels. Atherosclerosis 89: 109-116

[32] Rao DC, Laskarzewski PM, Morrison JA, et al. (1982) The Cincinnati Lipid Research Clinic family study: cultural and biological determinants of lipids and lipoprotein concentrations. Am J Hum Genet 34: 888-903

[33] Hamsten A, Iselius L, Dahlen G, de Faire U (1986) Genetic and cultural inheritance of serum lipids, low and high density lipoprotein cholesterol and serum apolipoproteins A-I, A-II and B. Atherosclerosis 60: 199-208

[34] Suzuki A, Lindor K, St Saver J, et al. (2005) Effect of changes on body weight and lifestyle in nonalcoholic fatty liver disease. J Hepatol 43: 1060-1066

[35] Petersen KF, Dufour S, Befroy D, Lehrke M, Hendler RE, Shulman GI (2005) Reversal of nonalcoholic hepatic steatosis, hepatic insulin resistance, and hyperglycemia by moderate weight reduction in patients with type 2 diabetes. Diabetes 54: 603-608

[36] Allayee H, Castellani LW, Cantor RM, de Bruin TW, Lusis AJ (2003) Biochemical and genetic association of plasma apolipoprotein A-II levels with familial combined hyperlipidemia. Circ Res 92: 1262-1267

[37] Adeyemo A, Luke A, Cooper R, et al. (2003) A genome-wide scan for body mass index among Nigerian families. Obes Res 11: 266-273

[38] Chen W, Li S, Cook NR, et al. (2004) An autosomal genome scan for loci influencing longitudinal burden of body mass index from childhood to young adulthood in white sibships: The Bogalusa Heart Study. Int J Obes Relat Metab Disord 28: 462-469

[39] Heijmans BT, Beem AL, Willemsen G, Posthuma D, Slagboom PE, Boomsma D (2004) Further evidence for a QTL influencing body mass index on chromosome 7p from a genome-wide scan in Dutch families. Twin Res 7: 192-196

[40] Weiss LA, Pan L, Abney M, Ober C (2006) The sex-specific genetic architecture of quantitative traits in humans. Nat Genet 38: 218-222

[41] Ogawa Y, Murata Y, Nishioka A, Inomata T, Yoshida S (1998) Tamoxifen-induced fatty liver in patients with breast cancer. Lancet 351: 725

[42] Nemoto Y, Toda K, Ono M, et al. (2000) Altered expression of fatty acid-metabolizing enzymes in aromatase-deficient mice. J Clin Invest 105: 1819-1825

[43] Campos H, Walsh BW, Judge H, Sacks FM (1997) Effect of estrogen on very low density lipoprotein and low density lipoprotein subclass metabolism in postmenopausal women. J Clin Endocrinol Metab 82: 3955-3963 
[44] Lander E, Kruglyak L (1995) Genetic dissection of complex traits: guidelines for interpreting and reporting linkage results. Nat Genet 11: 241-247

[45] Pollex RL, Hegele RA (2005) Complex trait locus linkage mapping in atherosclerosis: time to take a step back before moving forward? Arterioscler Thromb Vasc Biol 25: 1541-1544

[46] Pollex RL, Hegele RA (2006) Longitudinal differences in familial combined hyperlipidemia quantitative trait loci. Arterioscler Thromb Vasc Biol 26: e120 


\section{Fatty liver based subgroups}

Fatty liver-based identification of two distinct hypertriglyceridemic subgroups in familial combined hyperlipidemia

Brouwers MC, van Greevenbroek MM Bilderbeek-Beckers MA, Robertus-Teunissen MG van der Kallen CJ, Stehouwer CD de Bruin TW

Metabolism. 2007; 56(10):1311-1317 


\begin{abstract}
The present study was conducted to investigate whether the fatty liver phenotype could be helpful in the identification of subgroups with distinct metabolic properties and lipid profiles within familial combined hyperlipidemia.

One hundred eighty-five FCHL family members participated in the current study; 38 subjects were found to be hypertriglyceridemic of whom $66 \%$ showed evidence of fatty liver, as measured with ultrasound.

A detailed comparison between the hypertriglyceridemic FCHL subjects with ( $\mathrm{n}=25)$ and without $(\mathrm{n}=13)$ fatty liver revealed that, despite very similar plasma triglycerides levels (3.5 versus $3.2 \mathrm{mmol} / \mathrm{L}$ in subjects with and without fatty liver, respectively), the fatty liver subgroup presented with significantly higher body mass index, visceral adipose tissue (ultrasound), insulin and alanine aminotransferase levels. Moreover, VLDL-subclass analysis showed that the VLDL2fraction of the fatty liver subgroup contained significantly less cholesterol and triglycerides $(\mathrm{p}=$ 0.02 for both parameters), which was likely explained by a decreased VLDL2 particle number, since VLDL2 apolipoprotein B levels tended to be lower $(\mathrm{p}=0.08)$.

These data indicate that hypertriglyceridemic FCHL subjects may belong to metabolically distinct subgroups and suggest that a refinement of the hypertriglyceridemic FCHL phenotype by adding information on fatty liver will eventually facilitate the elucidation of its complex genetic background.
\end{abstract}




\section{Introduction}

Familial combined hyperlipidemia (FCHL) is the most prevalent (1:100) genetic hyperlipidemia in western society [1] and is associated with a two to five fold increased risk to develop cardiovascular complications $[2,3]$. It is estimated that ten percent of the survivors of a premature myocardial infarction is affected with FCHL [1]. Recent studies have revealed that FCHL patients, similar to patients with type 2 diabetes mellitus, share many features of the metabolic syndrome [4]: insulin resistance [5], (visceral) obesity [6], hypertension [7], elevated plasma triglycerides, low HDL-cholesterol and abundance of small-dense LDL particles [8].

We recently reported that another aspect of the metabolic syndrome, i.e. fatty liver, is also highly prevalent in FCHL [9,10], which is in part explained by an increased genetic susceptibility [10]. A recent study in normal subjects and patients with type 2 diabetes mellitus demonstrated that an increased amount of hepatic fat is associated with the overproduction of VLDL-particles [11]. This is of interest considering that VLDL-overproduction is one of the best-documented features of FCHL $[12,13]$. It is very likely that a similar relation is present in FCHL, given our previously reported relation between fatty liver and the amount of VLDL-particles in plasma [9].

However, FCHL is a complex, heterogeneous entity. It is anticipated that the hyperlipidemic state in FCHL is the consequence of not only the fatty liver-VLDL-overproduction pathway, but also of an impaired catabolism, e.g. a delayed remnant particle clearance [14].

Therefore, the aim of the present study was to explore whether it is possible to refine the FCHL phenotype, in particular the hypertriglyceridemic phenotype, by employing a relatively easily obtainable trait that is closely related to VLDL-overproduction, i.e. fatty liver. The primary goal was to evaluate whether it is possible to identify subjects with as well as subjects without fatty liver within the hypertriglyceridemic subgroup, since this would implicate that there are indeed distinct subgroups within the hypertriglyceridemic FCHL phenotype. For this we performed ultrasound scanning of the liver in our well-defined FCHL families and first determined the prevalence of hypertriglyceridemia in spouses and FCHL family members with and without radiological evidence of fatty liver. Subsequently, subgroups were identified within the hypertriglyceridemic phenotype based on the presence or absence of fatty liver to investigate whether differences in metabolic parameters and VLDL1 and VLDL2 particle properties could be discerned.

\section{Methods}

\section{Subjects}

FCHL probands $(\mathrm{n}=23)$, their relatives $(\mathrm{n}=162)$ and spouses $(\mathrm{n}=72)$ participated in this study. FCHL families were diagnosed as described previously [7]. Because FCHL family members and their spouses share a similar environment, observed differences are likely to be explained by genetic factors. It should, however, be noted that the spouses do not necessarily represent a random sample from the general population.

Subjects visited the research ward after an overnight fast, three days abstinence from alcohol and two weeks withdrawal from lipid lowering medication. Furthermore, subjects consumed no 
more than $20 \mathrm{~g}$ of alcohol daily, had a stable weight and did not take any medication associated with the development of fatty liver [15]. One subject was found to be seropositive for hepatitis $\mathrm{C}$ and was therefore excluded from this study.

The study protocol was approved by Human Investigations Review Committee at Maastricht University/Academic Hospital Maastricht. All subjects gave written informed consent.

\section{Anthropometric measurements}

Subjects were weighed in their underwear. Height was measured with a stadiometer and body mass index (BMI) was calculated as weight $(\mathrm{kg})$ divided by height $(\mathrm{m})$ squared. Waist circumference was determined in supine position at the level midway between lower rib and iliac crest.

\section{Ultrasound}

All ultrasound measurements were performed by the same researcher with an ATL 9 HDI ultrasound (US) system (Bothel, USA), as described previously [9]. Both visceral adipose tissue (VAT-US) and subcutaneous adipose tissue (SAT-US) thickness were determined at the same level as waist circumference. SAT-US was measured at the midline as the distance between skin and linea alba, using a L5-10 transducer. VAT-US was determined as the distance between the anterior of the vertebrate body and the peritoneum, exactly as described by Stolk et al [16]. All measurements were done with minimal pressure exerted on the probe and at the end of a normal expiration. The intra-observer variability, as determined in 30 subjects, was $1.7 \%$.

The presence of fatty liver was also detected by ultrasound, using a C7-4 and C4-2 transducer. Standardized images of the liver and right kidney were recorded on videotape and examined by an independent radiologist unaware of the subject's clinical characteristics. Diagnosis of fatty liver was established by conventional criteria, i.e. increased echogeneity ('bright liver'), posterior beam attenuation and decreased visualization of hepatic blood vessels [17, 18]. The different stages of fatty liver, i.e. mild, moderate and severe, have been described in detail elsewhere [9]. The intra-observer agreement expressed as kappa, determined in 30 random scans, was substantial $(x=0.74)$, which is in agreement with earlier studies [18].

\section{Laboratory measurements}

Blood was collected in pre-cooled EDTA tubes. After centrifugation at $3000 \mathrm{rpm}$ for $15 \mathrm{~min}$ at $4^{\circ} \mathrm{C}$, plasma was stored at $-80^{\circ} \mathrm{C}$ for subsequent analyses. Measurements of plasma triglycerides, total cholesterol, glucose, insulin and apolipoprotein B were done as previously described [19]. Alanine aminotransferase (ALT) levels were measured with a commercially available assay (Ecoline ${ }^{\circledR} \mathrm{S}+$, DiaSys Diagnostic Systems GmbH, Germany). The degree of insulin resistance was estimated with the homeostasis model assessment (HOMA-IR: glucose*insulin/22.5) [20]. VLDL isolation was done as described by Redgrave et al. [21]. Subsequently, VLDL1 and VLDL2 subfractions were separated by density gradient ultracentrifugation as described by Zhao et al [22], with minor modifications, which represents ultracentrifugation at $160000 \mathrm{~g}$ for 2.5 hours at $4^{\circ} \mathrm{C}$ in a SW40 Ti rotor. Collection of fractions started from the top of the tube, where the upper $1.5 \mathrm{~mL}$ represents VLDL1 and the lower $5 \mathrm{~mL}$ represents VLDL2. Cholesterol and triglycerides concentrations in the VLDL1 and VLDL2 fractions were measured similarly as in total plasma. 
Apolipoprotein B concentration in these fractions was quantified by gel electrophoresis according to the method of Karpe et al. [23].

\section{Statistical analyses}

Linear regression analyses were conducted to detect differences in general characteristics between FCHL probands, their relatives and spouses with correction for age and sex. The different subgroups entered the models as dummy variables. Logistic regression was used in case of dichotomous variables (e.g. fatty liver yes/no).

Differences in prevalence of hypertriglyceridemia between spouses and FCHL family members (i.e. probands and relatives combined) with and without fatty liver were tested with a chi-square test.

General characteristics and lipoprotein particle properties were compared between hypertriglyceridemic FCHL subjects with and without fatty liver by means of linear regression, with correction for age and sex. All analyses were conducted with SPSS 13.0 statistical package (SPSS Inc., Chicago, Ill.)

\section{Results}

\section{Population characteristics}

Characteristics of FCHL probands, their relatives and spouses are summarized in Table 1. Both FCHL probands and their relatives were more (abdominally) obese than their spouses, which was predominantly explained by an increased amount of visceral fat, as reflected by VAT-US. SATUS, measured at the same level as waist circumference, was not significantly different among the three groups. Plasma triglycerides and total cholesterol are by definition elevated in FCHL probands and were also significantly higher in their relatives in comparison with spouses. In addition, both probands and relatives exhibited higher liver-specific ALT levels and were more insulin-resistant than spouses, as suggested by the increased insulin and HOMA-IR levels. The prevalence of fatty liver, as estimated with ultrasound, was two times higher in both probands and relatives.

\section{Relation between fatty liver and hypertriglyceridemia in spouses and FCHL}

Numerous studies have shown that hepatic fat accumulation is associated with higher plasma triglyceride levels [24-26]. The increased prevalence of hypertriglyceridemia among spouses and FCHL family members with fatty liver is in concordance with these studies: only 1 out of 58 $(2 \%)$ spouses without ultrasonographical evidence of fatty liver had elevated plasma triglycerides above $2.3 \mathrm{mmol} / \mathrm{L}$, whereas hypertriglyceridemia was observed in 5/14 (36\%) of the spouses with fatty liver (Odds ratio [OR]: 31.7, 95\%CI: 3.3 - 303.2; p $<0.001$, Figure 1). Similarly, hypertriglyceridemia was present in 13/117 (11\%) FCHL family members without fatty liver (probands and relatives combined), in contrast to 25/68 (37\%) FCHL family members with fatty liver (OR: 4.7, 95\%CI: $2.2-9.9 ; \mathrm{p}<0.001$, Figure 1).

Of more interest, when spouses and FCHL family members without fatty liver were compared, the proportion of subjects who were hypertriglyceridemic was significantly higher in FCHL 
Table 1.

Population characteristics

\begin{tabular}{lccc}
\hline & Spouses & FCHL relatives & FCHL probands \\
\hline Male / female & $33 / 39$ & $87 / 75$ & $11 / 12$ \\
Age, years & $51.2 \pm 11.8$ & $45.6 \pm 15.6^{*}$ & $54.7 \pm 9.5 \dagger$ \\
& & & \\
BMI, kg/m ${ }^{2}$ & $24.6(22.0-28.1)$ & $25.6(22.9-28.7) \ddagger$ & $27.3(25.6-29.5) \ddagger$ \\
Waist circumference, cm & $91.1 \pm 12.7$ & $93.3 \pm 12.6 \ddagger$ & $99.0 \pm 7.8 \ddagger$ \\
SAT-US, cm & $2.3 \pm 1.1$ & $2.5 \pm 1.2$ & $2.8 \pm 1.1$ \\
VAT-US, cm & $7.2(5.6-9.3)$ & $7.4(6.0-9.5) \ddagger$ & $8.4(7.5-10.8) \ddagger$ \\
& & & \\
Triglycerides, mmol/L & $1.1(0.9-1.2)$ & $1.3(1.0-1.9) \ddagger$ & $3.2(1.9-4.0) \ddagger^{\wedge}$ \\
Total cholesterol, mmol/L & $5.4(4.7-5.9)$ & $5.5(4.6-6.4) \ddagger$ & $7.0(5.7-8.7) \ddagger^{\wedge}$ \\
Apolipoprotein B, g/L & $1.0 \pm 0.2$ & $1.1 \pm 0.3 \ddagger$ & $1.3 \pm 0.3 \ddagger$ \\
& & & \\
Insulin, U/L & $5.1(2.0-8.9)$ & $6.7(3.4-10.9) \ddagger$ & $9.6(7.7-14.9) \ddagger^{\wedge}$ \\
HOMA-IR & $1.0(0.5-2.1)$ & $1.5(0.7-2.5) \ddagger$ & $2.7(1.8-4.0) \ddagger^{\wedge}$ \\
& & & \\
ALT, U/L & $16.0(13.2-19.1)$ & $18.2(13.9-25.3) \ddagger$ & $24.0(17.2-28.4)^{\wedge}$ \\
Fatty liver, \% & 19 & $36 \ddagger$ & $43 \ddagger$
\end{tabular}

Data are presented as mean \pm SD or as median (interquartile range).

$* \mathrm{p}<0.05$ versus spouses, linear regression

t $\mathrm{p}<0.05$ versus FCHL relatives, linear regression

$\ddagger \mathrm{p}<0.05$ versus spouses, linear regression with correction for age and sex

${ }^{\mathrm{p}}<0.05$ versus FCHL relatives, linear regression with correction for age and sex

All analyses are Hochberg corrected

$(13 / 117[11 \%])$ than in spouses $(1 / 58[2 \%] ; p=0.03$, Figure 1$)$. The prevalence of hypertriglyceridemia did not differ between spouses and FCHL family members with fatty liver $(5 / 14[36 \%]$ versus $25 / 68[37 \%], p=0.94$, Figure 1$)$. Similar trends were observed when the data were stratified by sex (results not shown).

In our further analyses, we concentrated on the hypertriglyceridemic (HTG) FCHL subgroups with fatty liver $(\mathrm{n}=25)$ and without fatty liver $(\mathrm{n}=13)$ to investigate whether distinct properties could be discerned. 


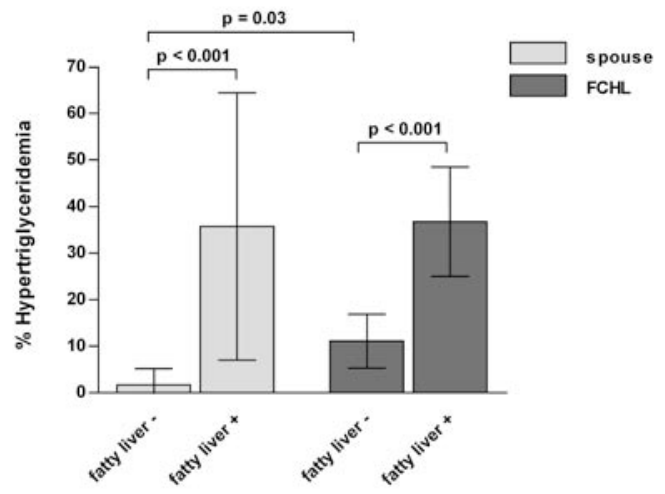

Figure 1.

Relation of fatty liver with the prevalence of hypertriglyceridemia in spouses ( $\mathrm{n}=58$ without fatty liver, $\mathrm{n}=14$ with fatty liver) and FCHL family members (probands and relatives combined; $\mathrm{n}=117$ without fatty liver, $\mathrm{n}=68$ with fatty liver). Error bars represent $95 \%$ confidence intervals.

Analyzed with chi-square tests. Hochberg corrected.

Characteristics of hypertriglyceridemic FCHL subjects with and without fatty liver

The characteristics of both HTG groups with and without fatty liver are displayed in Table 2. Of all subjects with fatty liver, 8 presented with mild fatty liver, 9 with moderate fatty liver and 8 with severe fatty liver. HTG FCHL subjects without fatty liver were only moderately overweight, in contrast to the HTG FCHL subjects with fatty liver who were, on average, obese $(\mathrm{p}<0.001)$. The amount of visceral fat was significantly higher in the HTG FCHL subjects with fatty liver, whereas no differences were observed for the amount of abdominal subcutaneous fat. Of interest, plasma triglycerides were very similar between both groups (Table 2) and, although the median values (but not the interquartile ranges) of total cholesterol and apolipoprotein B appeared to be different between the two groups (Table 2), age and gender corrected analyses revealed no significant differences $(\mathrm{p}=0.62$ and $\mathrm{p}=0.18$, respectively). HOMA-IR and plasma insulin and ALT levels were significantly higher in HTG FCHL subjects with fatty liver.

Of note, the FCHL subjects with and without fatty liver were derived from 17 and 10 different pedigrees, respectively, indicating that genetic dependency is not likely to account for the observed differences. 
Table 2.

Characteristics of hypertriglyceridemic FCHL patients with and without fatty liver

\begin{tabular}{lcc}
\hline & Fatty liver absent & Fatty liver present \\
\hline Male / female & $8 / 5$ & $10 / 15$ \\
Age, years & $53.0(43.5-54.5)$ & $55.0(45.0-59.5)$ \\
& & \\
Body mass index, kg/m² & $26.3(24.4-28.4)$ & $30.1(28.7-32.5)^{*}$ \\
Waist circumference, cm & $96.2(90.1-104.0)$ & $104.7(95.4-106.5)^{*}$ \\
SAT-US, cm & $2.9(2.4-3.5)$ & $3.1(2.1-3.8)$ \\
VAT-US, cm & $8.4(7.7-9.3)$ & $10.7(9.7-12.0)^{*}$ \\
& $3.2(3.1-3.8)$ & $3.5(2.7-4.9)$ \\
Triglycerides, mmol/L & $6.9(5.4-7.5)$ & $5.8(5.5-8.7)$ \\
Total cholesterol, mmol/L & $1.4(1.0-1.5)$ & $1.1(1.0-1.4)$ \\
Apolipoprotein B, g/L & & $12.8(9.4-17.7)^{*}$ \\
& $8.1(6.5-12.4)$ & $3.0(2.0-4.8)^{*}$ \\
Insulin, mU/L & $2.2(1.5-3.7)$ & $31.9(21.2-40.7)^{*}$ \\
HOMA-IR & $22.4(17.2-26.5)$ & \\
ALT, U/L & & \\
\hline
\end{tabular}

Data are expressed as median (interquartile range).

$* \mathrm{p}<0.05$, linear regression with correction for age and sex

Lipoprotein particle properties in hypertriglyceridemic FCHL subjects with and without fatty liver The production of VLDL1- and VLDL2-particles, the main constituents of plasma triglycerides, is, among others, driven by the amount of fat in the liver [11]. The current study also showed an association of fatty liver with hypertriglyceridemia. However, a FCHL subgroup had been identified with hypertriglyceridemia despite the absence of fatty liver. We realized that hypertriglyceridemia in absence of fatty liver could represent a different causality and we asked whether this could correspond to differences in lipoprotein amounts and composition, in particular VLDL-particles. Therefore, we determined the concentrations of triglycerides, cholesterol and apolipoprotein B in the VLDL1- and VLDL2-fraction in the HTG FCHL subgroups with and without fatty liver. Eight randomly selected normotriglyceridemic FCHL family members without fatty liver were included as a reference group (mean triglycerides: 1.2 $\mathrm{mmol} / \mathrm{L}$, total cholesterol: $5.6 \mathrm{mmol} / \mathrm{L}$, apolipoprotein B: $1.1 \mathrm{~g} / \mathrm{L}$ ).

As shown in Figure 2, both HTG subgroups showed increased concentrations of triglycerides and cholesterol in both the VLDL1- and VLDL2-fraction in comparison with the reference group. This was likely caused by an increased amount of particles because apolipoprotein B concentrations were generally increased in both fractions.

VLDL1 apolipoprotein B, cholesterol and triglycerides concentrations were not significantly 
different between both HTG groups (Figure 2). In contrast, in the VLDL2 fraction, both triglycerides and cholesterol concentrations were significantly higher in the HTG subgroup without fatty liver compared to the HTG FCHL subjects with fatty liver $(p=0.02$ for both parameters, Table 2). Since VLDL2 apolipoprotein B concentrations tended to be higher in the HTG subjects without fatty liver $(\mathrm{p}=0.08)$ and the composition of the particles was not different $(\mathrm{p}=0.58$ for VLDL2 triglycerides/apolipoprotein $\mathrm{B}$ ratio, $\mathrm{p}=0.88$ for VLDL2 cholesterol/apolipoprotein B ratio), it is likely that an increased VLDL2 particle number, rather than an increased particle size, accounts for the significantly higher VLDL2 triglycerides and cholesterol.
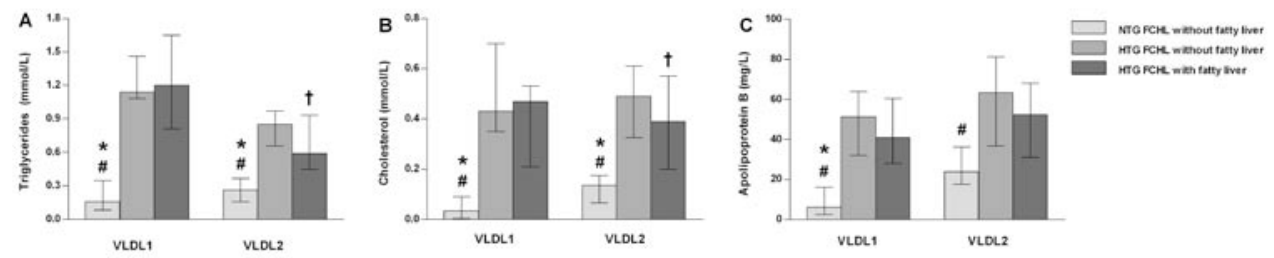

Figure 2.

Concentrations of triglycerides (panel A), cholesterol (panel B) and apolipoprotein B (panel C) in VLDL1 and VLDL2 fractions in normotriglyceridemic (NTG) FCHL family members without fatty liver $(\mathrm{n}=8)$, hypertriglyceridemic (HTG) FCHL subjects without fatty liver $(n=13)$ and hypertriglyceridemic FCHL subjects with fatty liver $(n=25)$. Median values are displayed. Error bars represent interquartile range.

$* \mathrm{p}<0.01$, normotriglyceridemic relatives vs hypertriglyceridemic FCHL without fatty liver;

$\#$ p $<0.01$, normotriglyceridemic relatives vs hypertriglyceridemic FCHL with fatty liver;

t $\mathrm{p}<0.05$ hypertriglyceridemic FCHL without fatty liver vs hypertriglyceridemic FCHL with fatty liver

Analyzed with linear regression, with correction for age and sex. Hochberg corrected.

\section{Discussion}

The heterogeneous nature of the dyslipidemia that is characteristic of familial combined hyperlipidemia (FCHL) has hampered researchers in their work to unravel its complex genetic background. The present study was conducted to investigate whether the application of the fatty liver trait could result in the identification of more homogeneous subgroups within FCHL and hence in a refinement of the FCHL phenotype. The study was triggered by the recent observation of Adiels et al. [11], who showed that the production of VLDL-particles, a process that is increased in FCHL $[12,13]$, is driven by the amount of fat that is accumulated in the liver. Furthermore, our laboratory recently demonstrated that the amount of hepatic fat is related with the amount of VLDL-particles in plasma in FCHL [9].

The prevalence of hypertriglyceridemia was associated with the presence of fatty liver in both FCHL family members and spouses, analogous to other reports [24-26]. However, several observations clearly distinguished FCHL family members from spouses. First, although the 
prevalence of hypertriglyceridemia was similar in spouses and FCHL family members when fatty liver was present, fatty liver itself was twice as prevalent in FCHL probands and their relatives in comparison to spouses. In other words: FCHL family members appear to be more prone to develop fatty liver than spouses, but as soon as fatty liver is present, the risk of developing hypertriglyceridemia is very similar. This observation further emphasizes the central role of fatty liver in the expression of FCHL, as recently reported $[9,10]$. Second, the prevalence of hypertriglyceridemia was significantly higher in those FCHL family members without fatty liver than in spouses without evidence of hepatic fat accumulation, indicating that fatty liver is not a prerequisite for the development of hypertriglyceridemia in a subgroup of FCHL patients.

A direct comparison between the hypertriglyceridemic FCHL subjects with and without fatty liver suggested that the underlying mechanisms for elevated plasma triglycerides are different. The subgroup with fatty liver was more (viscerally) obese and insulin-resistant, and had higher ALT levels. These parameters have indeed been associated with hepatic fat accumulation [24, 26]. Interestingly, plasma triglycerides, a variable that has also been linked to fatty liver [24-26], was very similar between both hypertriglyceridemic subgroups. Since ALT levels were within normal range in both subgroups, it was ethically not acceptable to perform liver biopsies to investigate whether both subgroups also differ in the degree of inflammation or fibrosis.

Analysis of the VLDL1- and VLDL2-particle profiles, particles that mainly determine plasma triglycerides concentrations, revealed that the hypertriglyceridemic subgroup with fatty liver had less VLDL2-particles compared to the hypertriglyceridemic subgroup without fatty liver, as shown by lower triglycerides, cholesterol and a trend towards lower apolipoprotein B concentrations in the VLDL2-fraction. Of note, the VLDL2 cholesterol, triglycerides and apolipoprotein B levels were still substantially higher than in the normotriglyceridemic reference group.

Since only six spouses were found to be hypertriglyceridemic, it was not feasible to investigate whether the existence of HTG subgroups with and without fatty liver are specific for FCHL or similar outcomes could be obtained within the spouses, as well.

The current data support the existence of two metabolically distinct subgroups within the hypertriglyceridemic FCHL phenotype but studies e.g. using stable isotopes are needed to provide the exact mechanistic difference. Since both insulin resistance and hepatic fat accumulation have been associated with VLDL-overproduction [11, 27, 28], one can speculate that overproduction may be responsible for the hypertriglyceridemia in those FCHL patients with fatty liver. Of note, given the significantly higher HOMA-IR levels in this subgroup, one may suggest that increased insulin resistance is actually the discriminating factor between hypertriglyceridemic subjects with and without fatty liver. However, hepatic fat accumulation and insulin resistance are highly correlated and current literature suggests that the former may precede the latter [29, 30]. The underlying mechanism for the hypertriglyceridemia of the FCHL subgroup without fatty liver is however much less obvious and it can be speculated that mixed causes including delayed elimination of remnants are involved.

One can conclude that the current study has demonstrated that it is feasible to identify subgroups within the hypertriglyceridemic FCHL phenotype based on the presence or absence of radiological evidence of fatty liver. Not only researchers would benefit from the identification of more homogeneous subgroups within FCHL, but also clinicians could use the fatty liver trait 
to select for the most optimal treatment strategy for an individual FCHL patient. The discovery and development of new therapeutic agents that target fatty liver and its associated metabolic abnormalities $[31,32]$ are in that respect of particular interest.

\section{References}

[1] Goldstein JL, Schrott HG, Hazzard WR, Bierman EL, Motulsky AG (1973) Hyperlipidemia in coronary heart disease. II. Genetic analysis of lipid levels in 176 families and delineation of a new inherited disorder, combined hyperlipidemia. J Clin Invest 52: 1544-1568

[2] Austin MA, McKnight B, Edwards KL, et al. (2000) Cardiovascular disease mortality in familial forms of hypertriglyceridemia: A 20-year prospective study. Circulation 101: $2777-2782$

[3] Voors-Pette C, de Bruin TW (2001) Excess coronary heart disease in Familial Combined Hyperlipidemia, in relation to genetic factors and central obesity. Atherosclerosis 157: 481-489

[4] Carr MC, Brunzell JD (2004) Abdominal obesity and dyslipidemia in the metabolic syndrome: importance of type 2 diabetes and familial combined hyperlipidemia in coronary artery disease risk. J Clin Endocrinol Metab 89: 2601-2607

[5] Aitman TJ, Godsland IF, Farren B, Crook D, Wong HJ, Scott J (1997) Defects of insulin action on fatty acid and carbohydrate metabolism in familial combined hyperlipidemia. Arterioscler Thromb Vasc Biol 17: 748-754

[6] Purnell JQ, Kahn SE, Schwartz RS, Brunzell JD (2001) Relationship of insulin sensitivity and ApoB levels to intra-abdominal fat in subjects with familial combined hyperlipidemia. Arterioscler Thromb Vasc Biol 21: 567-572

[7] Keulen ET, Voors-Pette C, de Bruin TW (2001) Familial dyslipidemic hypertension syndrome: familial combined hyperlipidemia, and the role of abdominal fat mass. Am J Hypertens 14: 357-363

[8] Ayyobi AF, McGladdery SH, McNeely MJ, Austin MA, Motulsky AG, Brunzell JD (2003) Small, Dense LDL and Elevated Apolipoprotein B Are the Common Characteristics for the Three Major Lipid Phenotypes of Familial Combined Hyperlipidemia. Arterioscler Thromb Vasc Biol 23: 1289-1294

[9] Brouwers MC, Bilderbeek-Beckers MA, Georgieva AM, van der Kallen CJ, van Greevenbroek MM, de Bruin TW (2007) Fatty liver is an integral feature of familial combined hyperlipidaemia: relationship with fat distribution and plasma lipids. Clin Sci (Lond) 112: 123-130

[10] Brouwers MC, Cantor RM, Kono N, et al. (2006) Heritability and genetic loci of fatty liver in familial combined hyperlipidemia. J Lipid Res 47: 2799-2807

[11] Adiels M, Taskinen MR, Packard C, et al. (2006) Overproduction of large VLDL particles is driven by increased liver fat content in man. Diabetologia: 1-11

[12] Venkatesan S, Cullen P, Pacy P, Halliday D, Scott J (1993) Stable isotopes show a direct relation between VLDL apoB overproduction and serum triglyceride levels and indicate a 
metabolically and biochemically coherent basis for familial combined hyperlipidemia. Arterioscler Thromb 13: 1110-1118

[13] Kissebah AH, Alfarsi S, Adams PW (1981) Integrated regulation of very low density lipoprotein triglyceride and apolipoprotein-B kinetics in man: normolipemic subjects, familial hypertriglyceridemia and familial combined hyperlipidemia. Metabolism 30: $856-868$

[14] Cabezas MC, de Bruin TW, Jansen H, Kock LA, Kortlandt W, Erkelens DW (1993) Impaired chylomicron remnant clearance in familial combined hyperlipidemia. Arterioscler Thromb 13: 804-814

[15] Farrell GC (2002) Drugs and steatohepatitis. Semin Liver Dis 22: 185-194

[16] Stolk RP, Wink O, Zelissen PM, Meijer R, van Gils AP, Grobbee DE (2001) Validity and reproducibility of ultrasonography for the measurement of intra-abdominal adipose tissue. Int J Obes Relat Metab Disord 25: 1346-1351

[17] Saverymuttu SH, Joseph AE, Maxwell JD (1986) Ultrasound scanning in the detection of hepatic fibrosis and steatosis. Br Med J (Clin Res Ed) 292: 13-15

[18] Saadeh S, Younossi ZM, Remer EM, et al. (2002) The utility of radiological imaging in nonalcoholic fatty liver disease. Gastroenterology 123: 745-750

[19] Keulen ET, Kruijshoop M, Schaper NC, Hoeks AP, de Bruin TW (2002) Increased intima media thickness in familial combined hyperlipidemia associated with apolipoprotein B. Arterioscler Thromb Vasc Biol 22: 283-288

[20] Matthews DR, Hosker JP, Rudenski AS, Naylor BA, Treacher DF, Turner RC (1985) Homeostasis model assessment: insulin resistance and beta-cell function from fasting plasma glucose and insulin concentrations in man. Diabetologia 28: 412-419

[21] Redgrave TG, Roberts DC, West CE (1975) Separation of plasma lipoproteins by density gradient ultracentrifugation. Anal Biochem 65: 42-49

[22] Zhao SP, Bastiaanse EM, Hau MF, et al. (1995) Separation of VLDL subfractions by density gradient ultracentrifugation. J Lab Clin Med 125: 641-649

[23] Karpe F, Hamsten A (1994) Determination of apolipoproteins B-48 and B-100 in triglyceride-rich lipoproteins by analytical SDS-PAGE. J Lipid Res 35: 1311-1317

[24] Westerbacka J, Corner A, Tiikkainen M, et al. (2004) Women and men have similar amounts of liver and intra-abdominal fat, despite more subcutaneous fat in women: implications for sex differences in markers of cardiovascular risk. Diabetologia 47: 1360-1369

[25] de Bruin TW, Georgieva AM, Brouwers MC, Heitink MV, van der Kallen CJ, van Greevenbroek MM (2004) Radiological evidence of nonalcoholic fatty liver disease in familial combined hyperlipidemia. Am J Med 116: 847-849

[26] Kelley DE, McKolanis TM, Hegazi RA, Kuller LH, Kalhan SC (2003) Fatty liver in type 2 diabetes mellitus: relation to regional adiposity, fatty acids, and insulin resistance. Am J Physiol Endocrinol Metab 285: E906-916

[27] Malmstrom R, Packard CJ, Caslake M, et al. (1997) Defective regulation of triglyceride metabolism by insulin in the liver in NIDDM. Diabetologia 40: 454-462

[28] Malmstrom R, Packard CJ, Watson TD, et al. (1997) Metabolic basis of 
hypotriglyceridemic effects of insulin in normal men. Arterioscler Thromb Vasc Biol 17: 1454-1464

[29] Westerbacka J, Lammi K, Hakkinen AM, et al. (2005) Dietary fat content modifies liver fat in overweight nondiabetic subjects. J Clin Endocrinol Metab 90: 2804-2809

[30] Petersen KF, Dufour S, Befroy D, Lehrke M, Hendler RE, Shulman GI (2005) Reversal of nonalcoholic hepatic steatosis, hepatic insulin resistance, and hyperglycemia by moderate weight reduction in patients with type 2 diabetes. Diabetes 54: 603-608

[31] Neuschwander-Tetri BA, Brunt EM, Wehmeier KR, Oliver D, Bacon BR (2003) Improved nonalcoholic steatohepatitis after 48 weeks of treatment with the PPAR gamma ligand rosiglitazone. Hepatology 38: 1008-1017

[32] Despres JP, Golay A, Sjostrom L (2005) Effects of rimonabant on metabolic risk factors in overweight patients with dyslipidemia. N Engl J Med 353: 2121-2134 


\section{Fatty liver based subgroups}

Inflammation, endothelial function and fibrinolysis in relation to fatty liver and hypertriglyceridemia in familial combined hyperlipidemia

Brouwers MC, Govers-Riemslag J Schalkwijk CG, van Greevenbroek MM van der Kallen CJ, Bekers O van Dieijen-Visser MP, ten Oever J Bilderbeek-Beckers MA de Bruin TW, ten Cate $\mathrm{H}$

Stehouwer CD

Thromb Res. 2007; in press 


\begin{abstract}
Familial combined hyperlipidemia (FCHL) is a genetic form of dyslipidemia, which is characterized by an increased cardiovascular risk. The current study was conducted to investigate the relation of endothelial, inflammatory and fibrinolysis markers with the presence of hypertriglyceridemia and fatty liver in FCHL, in order to advance insight in their contribution to the cardiovascular risk profile.

Key plasma markers of low-grade inflammation, endothelial dysfunction and fibrinolysis were measured in 38 hypertriglyceridemic FCHL patients and 38 age and sex-matched spouses. The presence of fatty liver was determined with ultrasound.

HsCRP, vWF, PAI-1, tPA and tPA/PAI-1 complex levels were significantly higher in hypertriglyceridemic FCHL patients compared to spouses $(\mathrm{p}<0.05)$. Subsequent analyses revealed that these increased levels were confined to FCHL patients with the fatty liver phenotype $(\mathrm{n}=25)$. Only PAI-1 and tPA levels were also elevated in the hypertriglyceridemic FCHL patients without fatty liver $(\mathrm{n}=13)$. Of interest, 11 hypertriglyceridemic non-FCHL patients with the E2/E2 genotype displayed significantly lower PAI-1 levels when compared to the overall FCHL population $(\mathrm{p}=0.001)$, implicating a role for apolipoprotein $\mathrm{E}$ in the relation of PAI-1 with plasma triglycerides.

In conclusion, markers of fibrinolysis were increased in all hypertriglyceridemic FCHL patients, whereas an increased state of endothelial dysfunction and inflammation was particularly observed in those hypertriglyceridemic FCHL patients who also have fatty liver. These results demonstrate the complex genesis of the unfavourable cardiovascular risk profile that is present in FCHL, and illustrate the potential risk of fatty liver above, and beyond hypertriglyceridemia per se.
\end{abstract}




\section{Introduction}

Familial combined hyperlipidemia (FCHL) was originally described as a highly prevalent familial dyslipidemia, characterized by different Fredrickson's subtypes of hyperlipidemia, among survivors of a premature myocardial infarction (1:10) [1]. It has been estimated that patients affected with FCHL are exposed to a two- to five-fold increased risk to develop a myocardial infarction [2, 3].

Elevated plasma LDL-cholesterol, apolipoprotein B and triglycerides levels, combined with reduced HDL-cholesterol concentrations and the presence of small-dense LDL-particles, probably all contribute to the increased cardiovascular risk in FCHL. Furthermore, most FCHL patients share many features of the metabolic syndrome, such as hypertension and visceral obesity $[4,5]$, factors that also confer an increased cardiovascular risk [6]. Other metabolic syndromerelated plasma markers that reflect activities such as low-grade inflammation [7], endothelial dysfunction [8] and decreased fibrinolysis [9], have been shown to be independently associated with an increased cardiovascular risk. Studies that reported on these emerging cardiovascular risk markers in FCHL did not yet differentiate between the different types of hyperlipidemia that are characteristic of FCHL [10-12]. This is of importance, given our previously observed marked difference in for example plasminogen activator inhibitor-1 (PAI-1) levels between hypercholesterolemic and hypertriglyceridemic FCHL patients [13], while the latter phenotype shows the closest correlation with the presence of metabolic syndrome [14].

We recently reported that an increased amount of hepatic fat appears to be the driving force behind the development of hypertriglyceridemia in most hypertriglyceridemic FCHL patients $[15,16]$. However, a substantial subgroup of approximately one-third of the hypertriglyceridemic FCHL patients did not have fatty liver, indicating that a different mechanism drives the hypertriglyceridemia in those subjects [16]. We questioned whether associations of FCHL with markers of inflammation, endothelial dysfunction and fibrinolysis differed according to the presence or absence of fatty liver in these patients.

Therefore, the objective of the current report was to study markers of low-grade inflammation (high sensitive $\mathrm{C}$-reactive protein [hsCRP] and soluble intercellular adhesion molecule 1 [sICAM1]), endothelial dysfunction (von Willebrand factor $[\mathrm{vWF}]$ and sE-selectin) and fibrinolysis (PAI-1, tissue plasminogen activator [tPA] and PAI1-tPA complex) in FCHL patients with hypertriglyceridemia, the lipid phenotype that is closely associated with the metabolic syndrome [14], and determine the effect of fatty liver on the observed relations.

\section{Materials and methods}

\section{Study population}

FCHL families were ascertained as described previously [4]. Hypertriglyceridemic (HTG) patients, defined as plasma triglycerides $>2.3 \mathrm{mmol} / \mathrm{L}$, were selected after extensive screening of 185 FCHL family members, as described in detail elsewhere [16]. Age and sex-matched spouses of FCHL family members were invited as a reference group.

Subjects visited the research clinic in the morning after an overnight fast and total abstinence 
from alcohol for at least the last three days. Lipid-lowering medication was withdrawn two weeks prior to the visit. Furthermore, subjects did not consume more than $20 \mathrm{~g}$ alcohol daily, had a stable weight and did not use any medication associated with development of fatty liver [17]. One subject was excluded because of hepatitis $\mathrm{C}$ seropositivity. Weight, height, waist circumference and blood pressure were measured as described previously [18].

The study protocol was approved by the Human Investigations Review Committee at Maastricht University/Academic Hospital Maastricht. All subjects gave written informed consent.

\section{Ultrasound measurements}

The amount of visceral adipose tissue (VAT-US) and the presence or absence of fatty liver were both determined with an ATL 9 HDI ultrasound system, as described in detail elsewhere [15, 19, 20].

\section{Laboratory measurements}

Blood was collected in pre-cooled EDTA containing tubes for measurements of plasma total cholesterol, HDL-cholesterol, triglycerides, insulin, hsCRP, sICAM-1 and sE-selectin, and in $3.8 \%$ sodium citrate tubes on room temperature for measurements of plasma PAI- 1 antigen, tPA antigen, PAI-1-tPA complex and vWF. Plasma triglycerides, total cholesterol, HDL-cholesterol, insulin, tPA antigen and $\mathrm{vWF}$ were determined as described before [4, 13]. sICAM and sEselectin were analyzed by a commercially available ELISA (Diaclone Research, France). HsCRP was determined on a BN ProSpect (Dade Behring, Germany) based on particle-enhanced immunonephelometry. The concentration of PAI-1 antigen and tPA/PAI-1 complexes were measured by commercial ELISA techniques (Technoclone, NL); Apo E polymorphisms were genotyped as described previously [21].

\section{Statistical analyses}

Data are presented as mean $\pm \mathrm{SD}$, or as median (interquartile range) in case of non-normal distribution. HTG FCHL patients and spouses were compared with a Student's T-test or a MannWhitney $U$ test in case of non-normal distribution. Differences between other groups, such as HTG FCHL patients with and without fatty liver, were analyzed with linear regression, with the variable of interest as dependent variable and the different groups and confounding factors as independent variables. All analyses were conducted with SPSS 13.0 statistical package.

\section{Results}

Markers of endothelial dysfunction, inflammation and fibrinolysis in spouses and bypertriglyceridemic FCHL patients

Table 1 shows the general descriptives of the 38 hypertriglyceridemic (HTG) FCHL patients and 38 spouses (matched for age and sex). The proportion of smokers was very comparable between FCHL patients and their spouses $(26 \%$ versus $22 \%)$, suggesting that lifestyle habits are indeed similar between both groups. As expected, HTG FCHL patients were more (viscerally) obese, as reflected by significantly higher BMI and VAT-US, and displayed significantly higher insulin 
levels, indicative of an insulin-resistant state. In addition, fatty liver was almost three times as prevalent in HTG FCHL patients compared to spouses.

Plasma hsCRP, vWF, PAI-1 antigen, tPA antigen and tPA/PAI-1 complex levels, were significantly higher in the HTG FCHL patients compared to spouses (Table 1). The significant difference in hsCRP levels between FCHL patients and spouses was completely abolished when adjusted for BMI or VAT-US ( $\mathrm{p}=0.58$ and $\mathrm{p}=0.52$, respectively). In contrast, the difference in PAI-1 levels remained after adjustment for BMI and VAT-US ( $p<0.001$ and $\mathrm{p}=0.001$, respectively). Further adjustment for endothelial markers (vWF or sE-selectin), total cholesterol, blood pressure or insulin levels did not affect the results for PAI-1 antigen levels either. Finally, the increased state of low-grade inflammation did not account for the increased levels of vWF in the HTG FCHL patients, since adjustment for sICAM-1 or CRP levels did not influence the results, nor did correction for insulin levels, (HDL-) cholesterol or systolic and diastolic blood pressure.

Table 1.

General characteristics and endothelial, inflammatory and fibrinolysis markers in HTG FCHL patients and spouses

\begin{tabular}{lcc}
\hline & Spouses & HTG FCHL patients \\
\hline Male / female, $\mathrm{n}$ & $17 / 21$ & $18 / 20$ \\
Age, years & $52.9 \pm 9.9$ & $52.1 \pm 9.6$ \\
Smoking, \% & $26 \%$ & $22^{\%}$ \\
Systolic blood pressure, $\mathrm{mmHg}$ & $133 \pm 20$ & $145 \pm 18^{*}$ \\
Diastolic blood pressure, $\mathrm{mmHg}$ & $84 \pm 10$ & $91 \pm 9^{*}$ \\
Body mass index, kg/m ${ }^{2}$ & $25.7 \pm 3.9$ & $29.4 \pm 3.6^{*}$ \\
VAT-US, cm & $7.6 \pm 2.6$ & $10.0 \pm 2.6^{*}$ \\
Insulin, mU/L & $5.6(2.0-9.9)$ & $11.8(7.6-16.3) \dagger$ \\
Total cholesterol, mmol/L & $5.2(4.5-5.9)$ & $6.3(5.4-8.2) \dagger$ \\
HDL-cholesterol, mmol/L & $1.0 \pm 0.2$ & $0.7 \pm 0.2^{*}$ \\
Triglycerides, mmol/L & $1.2(0.9-1.7)$ & $3.4(2.9-4.4) \dagger$ \\
Fatty liver, \% & 24 & $66 \neq$ \\
hsCRP, mg/L & $1.1(0.5-3.1)$ & $1.6(0.9-7.2) \dagger$ \\
sICAM-1, ng/ml & $485 \pm 145$ & $500 \pm 100$ \\
vWF, $\%$ & $109(78-143)$ & $132(94-167) \dagger$ \\
sE-selectin, ng/ml & $61.3 \pm 28.4$ & $69.7 \pm 27.6$ \\
PAI-1, ng/ml & $17.6(13.0-33.0)$ & $47.6(27.6-88.6) \dagger$ \\
tPA, ng/ml & $8.8 \pm 3.9$ & $14.1 \pm 3.1 *$ \\
tPA/PAI-1 complex, ng/ml & $24.6(7.2-46.1)$ & $48.1(34.5-57.8) \dagger$ \\
\hline
\end{tabular}

Data are presented as mean $\pm \mathrm{SD}$, or as median (interquartile range)

$* \mathrm{p}<0.05$, Student's T-test, $\uparrow \mathrm{p}<0.05$, Mann-Whitney U test, $\neq \mathrm{p}<0.001$, Pearson chi-square 
Markers of endothelial dysfunction, inflammation and fibrinolysis in hypertriglyceridemic FCHL patients with and without fatty liver

The hypertriglyceridemic FCHL population was subsequently divided based on the presence or absence of fatty liver. HTG FCHL patients with fatty liver were more (viscerally) obese and presented with higher insulin levels (Table 2). Of note, plasma triglycerides were not significantly different between both groups (Table 2).

All markers of endothelial dysfunction, inflammation and fibrinolysis (except tPA/PAI-1 complex) tended to be higher in the HTG subgroup with fatty liver, and the difference was significant for hsCRP, sE-selectin and PAI-1.

Again, adjustment for BMI or VAT-US abolished the results for hsCRP ( $p=0.76$ and $p=0.24$, respectively), but not completely for PAI-1 antigen levels ( $\mathrm{p}=0.06$ and $\mathrm{p}=0.06$, respectively). Adjustment for plasma insulin levels did not affect the results for PAI-1 $(p=0.03)$.

Of additional interest, plasma hsCRP, sICAM-1, vWF and sE-selectin levels appeared very similar in HTG FCHL patients without fatty liver and spouses, whereas all fibrinolysis markers were significantly higher in the former group ( $\mathrm{p}<0.05$, Mann-Whitney $\mathrm{U}$ test). PAI- 1 and tPA remained significantly different after adjustment for VAT-US or insulin levels, suggesting that hypertriglyceridemia per se may affect fibrinolysis in FCHL.

Table 2 .

General characteristics and markers of endothelial dysfunction, inflammation and fibrinolysis in hypertriglyceridemic FCHL patients with and without fatty liver

\begin{tabular}{lcc}
\hline & Fatty liver absent & Fatty liver present \\
\hline Male / female, $\mathrm{n}$ & $8 / 5$ & $10 / 15$ \\
Age, years & $53.0(43.5-54.5)$ & $55.0(45.0-59.5)$ \\
Smoking, \% & 18 & 24 \\
Systolic blood pressure, mmHg & $140(128-157)$ & $145(132-157)$ \\
Diastolic blood pressure, mmHg & $89(83-97)$ & $89(83-98)$ \\
Body mass index, kg/m ${ }^{2}$ & $26.3(24.4-28.4)$ & $30.1(28.7-32.5)^{*}$ \\
VAT-US, cm & $8.4(7.7-9.3)$ & $10.7(9.7-12.0)^{*}$ \\
Insulin, mU/L & $8.1(6.5-12.4)$ & $12.8(9.4-17.7)^{*}$ \\
Total cholesterol, mmol/L & $6.9(5.4-7.5)$ & $5.8(5.5-8.7)$ \\
HDL-cholesterol, mmol/L & $0.7(0.6-1.0)$ & $0.7(0.5-0.8)$ \\
Triglycerides, mmol/L & $3.2(3.1-3.8)$ & $3.5(2.7-4.9)$ \\
hsCRP, mg/L & $0.9(0.6-1.9)$ & $2.6(1.2-9.2)^{*}$ \\
sICAM-1, ng/ml & $459(414-529)$ & $514(434-584)$ \\
vWF, \% & $100(87-164)$ & $139(97-169)$ \\
sE-selectin, ng/ml & $58.0(35.0-66.0)$ & $71.0(55.5-99.5)^{*}$ \\
PAI-1, ng/ml & $34.0(26.3-46.1)$ & $64.1(33.1-112.9)^{*}$ \\
tPA, ng/ml & $12.1(11.2-15.7)$ & $15.2(13.3-16.6)$ \\
tPA/PAI-1 complex, ng/ml & $51.2(29.3-80.4)$ & $47.4(37.2-58.2)$ \\
\hline
\end{tabular}

Data are presented as medians (interquartile range), ${ }^{*} \mathrm{p}<0.05$, linear regression, sex adjusted. 


\section{Absence of PAI-1 antigen in the VLDL-fraction}

Subsequent experiments were performed to gain more insight in the contribution of plasma triglycerides to markers of fibrinolysis in FCHL. Since several coagulation proteins have been reported to have an increased affinity for triglyceride-rich lipoproteins [22], we questioned whether this was true for PAI-1, as well. Such an association could theoretically account for the relation between plasma triglycerides and PAI-1 levels.

We therefore isolated VLDL-particles by gradient ultracentrifugation [23] in three HTG FCHL patients and measured PAI-1 antigen levels in this fraction. However, PAI-1 antigen levels were not detectable in the VLDL-fraction, indicating that PAI-1 does not associate with VLDLparticles.

\section{Hypertriglyceridemic patients with E2/E2 genotype display lower PAI-1 antigen levels}

Previous in vitro studies have demonstrated that triglyceride-rich particles are able to induce the synthesis of PAI-1 in cultured endothelial and HepG2 cells, which is mediated by the interaction with apolipoprotein $\mathrm{B} / \mathrm{E}$ receptors $[24,25]$. To investigate the in vivo contribution of this process to plasma PAI-1 levels, we included a hypertriglyceridemic population that is characterized by a defective interaction between VLDL-particles and the apolipoprotein B/E receptor, i.e. E2/E2 homozygotes [26].

Eleven patients with E2/E2 genotype were recruited from our lipid clinic. Their characteristics are displayed in Table 3. A direct comparison with the HTG FCHL patients (similar as presented in Table 1) revealed that the E2/E2 sample contained significantly more men ( $p=0.01$, Fisher's exact test) whose HDL-cholesterol levels were significantly higher $(\mathrm{p}<0.001$, linear regression, sex-adjusted). Of importance, none of the other metabolic parameters, including plasma triglycerides, differed between both groups (sex adjusted, Table 3). Despite these comparable characteristics, plasma PAI-1 antigen levels were significantly lower in the patients with the E2/ E2 genotype ( $p=0.001)$, as shown in Figure 1, panel A. Similar trends were observed when both patient groups were stratified by the presence or absence of fatty liver (Figure 1, panel B and C). Indeed, correction for fatty liver did not affect the results. After further adjustment for VATUS, plasma triglycerides, HDL-cholesterol and insulin levels, patients with E2/E2 genotype still displayed 23\% lower plasma PAI-1 antigen levels than the HTG FCHL patients ( $\mathrm{p}<0.001)$. 
Table 3.

Characteristics of patients with E2/E2 genotype compared to hypertriglyceridemic FCHL patients

\begin{tabular}{lcc}
\hline & HTG FCHL & E2/E2 \\
\hline Male / female, $\mathrm{n}$ & $18 / 20$ & $10 / 1^{*}$ \\
Age, years & $52.1 \pm 9.6$ & $50.0 \pm 11.6$ \\
Smoking, \% & 22 & 27 \\
Systolic blood pressure, mmHg & $145 \pm 18$ & $134 \pm 16$ \\
Diastolic blood pressure, mmHg & $91 \pm 9$ & $87 \pm 7$ \\
Body mass index, kg/m2 & $29.4 \pm 3.6$ & $28.4 \pm 4.2$ \\
VAT-US, cm & $10.0 \pm 2.6$ & $10.7 \pm 2.8$ \\
Total cholesterol, mmol/L & $6.3(5.4-8.2)$ & $6.0(4.8-7.8)$ \\
HDL-cholesterol, mmol/L & $0.7 \pm 0.2$ & $0.9 \pm 0.2 \dagger$ \\
Triglycerides, mmol/L & $3.4(2.9-4.4)$ & $2.9(1.9-6.8)$ \\
Insulin, mU/L & $11.8(7.6-16.3)$ & $10.0(6.8-18.8)$ \\
Fatty liver, \% & 66 & 55 \\
\hline
\end{tabular}

Data are presented as mean $\pm \mathrm{SD}$ or as medians (interquartile range)

$* \mathrm{p}<0.05$, Fisher's exact test

$+\mathrm{p}<0.05$, linear regression, sex adjusted

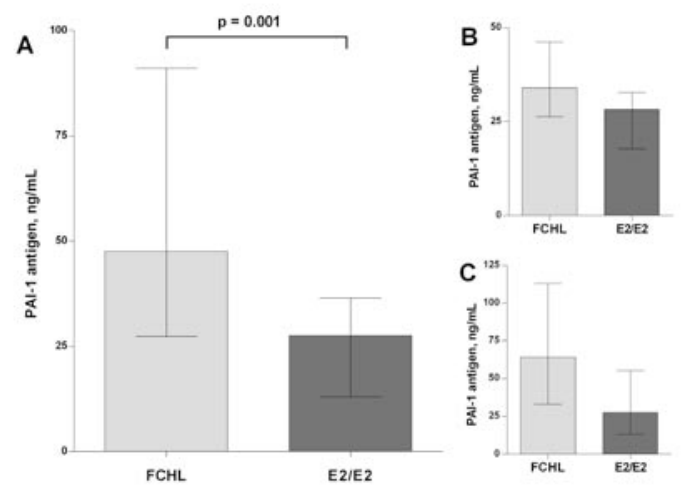

Figure 1.

Plasma PAI-1 antigen levels in HTG FCHL patients $(\mathrm{n}=38)$ versus patients with E2/E2 genotype $(\mathrm{n}=11)($ panel A). Panel B and C display the results stratified by the absence or presence of fatty liver, respectively. Bars represent medians with errror bars as the interquartile range. Analyzed with linear regression with adjustment for sex. 


\section{Discussion}

Familial combined hyperlipidemia is a highly prevalent disease characterized by an increased cardiovascular risk [1-3], which is accounted for by its typical dyslipidemia and its association with the metabolic syndrome. The present study was conducted to investigate markers of lowgrade inflammation (hsCRP and sE-selectin), endothelial dysfunction (vWF and sICAM) and impaired fibrinolysis (PAI-1, tPA and PAI-1-tPA complex) in hypertriglyceridemic patients with familial combined hyperlipidemia (FCHL), the phenotype closest associated with the metabolic syndrome [14], and determine the effect of fatty liver on the observed relations.

Hypertriglyceridemic FCHL patients presented with significantly higher hsCRP, vWF, PAI-1, tPA and PAI-1-tPA complex levels, indicating an impaired fibrinolysis and an increased state of lowgrade inflammation and endothelial dysfunction in comparison with their spouses. Other markers of low-grade inflammation and endothelial dysfunction, such as sICAM-1 and sE-selectin, were not significantly different between both groups, in line with the recent report of ter Avest et al [12]. In this respect it is of importance to note that the markers under investigation are not one hundred percent specific for their purpose, since their concentrations are not only dependent on production, but are also determined by clearance. Furthermore, tPA, PAI-1 and sICAM are also derived from endothelial cells and may therefore also reflect endothelial dysfunction, whereas sE-selectin and vWF also reflect, to some extent, low-grade inflammation [27].

Markers of low-grade inflammation and endothelial dysfunction were particularly elevated in those HTG patients with an increased amount of hepatic fat. HTG FCHL patients without radiological evidence of fatty liver did not display increased levels of inflammatory and endothelial markers when compared to spouses. The association of these markers with the amount of hepatic fat is in concordance with previous results obtained from non-diabetic, non-FCHL subjects with fatty liver [28] and supports cross-sectional and prospective data that nonalcoholic fatty liver disease associates with an increased cardiovascular risk [29-31]. Half of the hypertriglyceridemic FCHL patients with fatty liver displayed hsCRP levels above $3.0 \mathrm{mg} / \mathrm{L}$, the threshold value that is associated with an increased cardiovascular risk [32]. Although CRP is secreted by the liver, it is questionable whether hepatic fat accumulation by itself causes an increased secretion of this inflammation marker. Given the completely abolished association of hsCRP with fatty liver after adjustment for (visceral) obesity, it is more likely that the amount of adipose tissue determines plasma hsCRP levels, probably by means of increased release of interleukin-6, the main mediator of CRP-secretion [33]. In contrast, adjustment for (visceral) obesity or insulin resistance hardly affected the association of PAI-1 with fatty liver, suggesting an active contribution of fatty liver to increased PAI-1 levels and thus to an unfavourable cardiovascular risk profile, which is in concordance with recent cross-sectional and interventional studies [34, 35].

The current study supports division of the heterogeneous HTG population into more homogeneous subgroups, for example by employing the fatty liver trait, since these subgroups appear to have different cardiovascular risk profiles. This illustrates the complex genesis of 
the unfavourable cardiovascular risk profile in FCHL. It should however be recognized that HTG FCHL patients without fatty liver did not present with a normal risk profile. Although the state of low-grade inflammation and endothelial dysfunction was similar to spouses, we showed that blood pressure, plasma lipids, fibrinolysis markers were still significantly higher, the latter even after correction for (visceral) obesity or insulin resistance. We therefore postulate that plasma triglycerides might have a direct effect on plasma PAI-1 levels in HTG FCHL patients. In vivo evidence for this assumption has been provided by intravenous triglycerides infusion experiments in healthy men [36]. We showed that this is not the consequence of accumulation of PAI-1 in plasma due to an affinity for VLDL-particles, as has been observed for several vitamin K-dependent coagulation factors [22], since PAI-1 was not be detectable in VLDLparticles isolated by ultracentrifugation.

In vitro studies have shown that VLDL-particles stimulate PAI-1 secretion by endothelial and HepG2 cells, a process that is dependent on the interaction of VLDL-particles with the apo $\mathrm{B} / \mathrm{E}$ receptor $[24,25]$. We are the first to report the relevance of this process to variations in plasma PAI-1 levels, in vivo. Patients with the E2/E2 genotype (familial dysbetalipoproteinemia), who are known to have a defective binding to the apo B/E receptor [26], displayed $20 \%$ lower PAI-1 levels than HTG FCHL patients. Potential confounders of this observation, such as visceral obesity, insulin resistance, plasma triglycerides and presence of fatty liver were very similar between HTG FCHL patients and patients with E2/E2 genotype. Of note, none of the HTG FCHL patients carried two $\varepsilon 2$ alleles, since the E2/E2 genotype is an exclusion criterion of FCHL [1, 4]. At first sight, our results may appear to be in contrast with a previous genetic association study that did not report an association of PAI-1 levels with the 82 alleles [37]. However, that study population consisted of only E2-heterozygotes, but no E2-homozygotes [37], which may have masked any genotypic influence.

The current sample of HTG FCHL patients has originated from an extensive screening procedure in our well-defined FCHL population, as described elsewhere [16]. In addition, patients with familial dysbetalipoproteinemia have always been difficult to collect given the low prevalence $(0.1 \%)$ of this disorder in the general population [38]. For these reasons, the present study was conducted in a relatively small sample of subjects, which might have caused type II statistical errors. We do however believe that this unique combination of two carefully phenotyped dyslipidemic populations has provided valuable information with regard to hepatic fat accumulation and hypertriglyceridemia in relation to cardiovascular risk markers in FCHL.

To summarize, we have demonstrated that elevation of inflammatory and endothelial markers is particularly confined to hypertriglyceridemic FCHL patient with fatty liver, whereas markers of fibrinolysis are also increased in hypertriglyceridemic patients without fatty liver. Apolipoprotein $\mathrm{E}$ is likely to play a role in this latter observation (summarized in Figure 2). The present study emphasizes the complex genesis of the unfavourable cardiovascular risk profile in FCHL. 


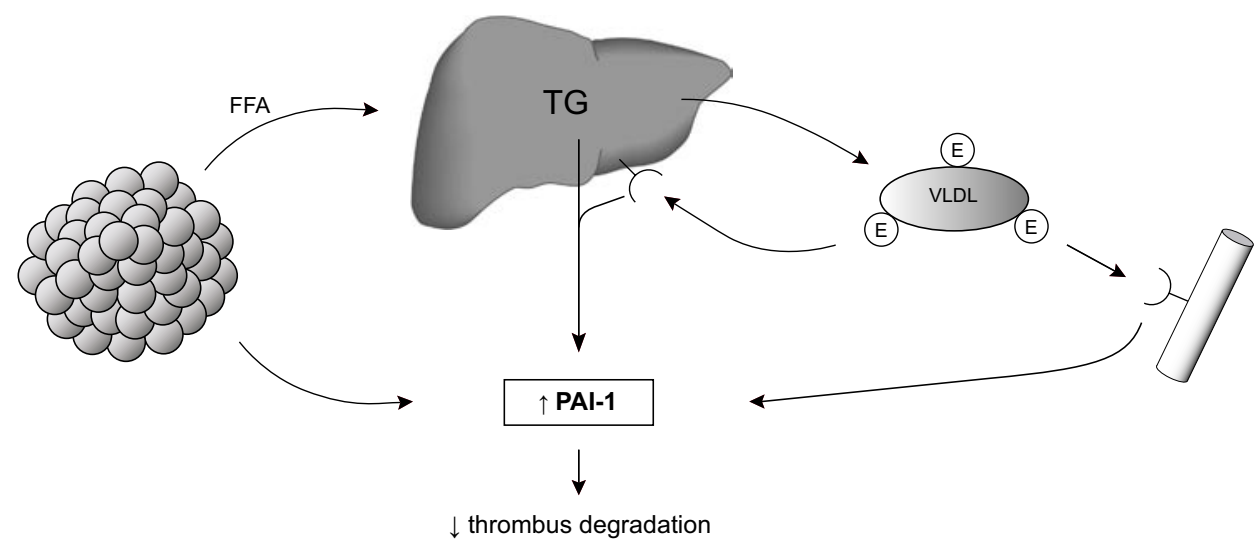

Figure 2.

Contribution of adipose tissue, liver and blood vessel to elevated plasma PAI-1 levels

\section{References}

[1] Goldstein JL, Schrott HG, Hazzard WR, Bierman EL, Motulsky AG (1973)

Hyperlipidemia in coronary heart disease. II. Genetic analysis of lipid levels in 176 families and delineation of a new inherited disorder, combined hyperlipidemia. J Clin Invest 52: 1544-1568

[2] Austin MA, McKnight B, Edwards KL, et al. (2000) Cardiovascular disease mortality in familial forms of hypertriglyceridemia: A 20-year prospective study. Circulation 101: $2777-2782$

[3] Voors-Pette C, de Bruin TW (2001) Excess coronary heart disease in Familial Combined Hyperlipidemia, in relation to genetic factors and central obesity. Atherosclerosis 157: 481-489

[4] Keulen ET, Voors-Pette C, de Bruin TW (2001) Familial dyslipidemic hypertension syndrome: familial combined hyperlipidemia, and the role of abdominal fat mass. Am J Hypertens 14: 357-363

[5] Purnell JQ, Kahn SE, Schwartz RS, Brunzell JD (2001) Relationship of insulin sensitivity and ApoB levels to intra-abdominal fat in subjects with familial combined hyperlipidemia Arterioscler Thromb Vasc Biol 21: 567-572

[6] Kannel WB, Cupples LA, Ramaswami R, Stokes J, 3rd, Kreger BE, Higgins M (1991) Regional obesity and risk of cardiovascular disease; the Framingham Study. J Clin Epidemiol 44: 183-190

[7] Ridker PM, Rifai N, Rose L, Buring JE, Cook NR (2002) Comparison of C-reactive protein and low-density lipoprotein cholesterol levels in the prediction of first cardiovascular events. N Engl J Med 347: 1557-1565

[8] de Jager J, Dekker JM, Kooy A, et al. (2006) Endothelial dysfunction and low-grade inflammation explain much of the excess cardiovascular mortality in individuals with type 
2 diabetes: the Hoorn Study. Arterioscler Thromb Vasc Biol 26: 1086-1093

[9] Hamsten A, Wiman B, de Faire U, Blomback M (1985) Increased plasma levels of a rapid inhibitor of tissue plasminogen activator in young survivors of myocardial infarction. $\mathrm{N}$ Engl J Med 313: 1557-1563

[10] Stroes E, de Bruin T, de Valk H, et al. (1997) NO activity in familial combined hyperlipidemia: potential role of cholesterol remnants. Cardiovasc Res 36: 445-452

[11] Karasek D, Vaverkova H, Halenka M, Budikova M, Novotny D (2005) Soluble cell adhesion molecules s-VCAM-1 and s-ICAM-1 in subjects with familial combined hyperlipidemia. Biomed Pap Med Fac Univ Palacky Olomouc Czech Repub 149: 101-108

[12] Ter Avest E, Holewijn S, van Tits LJ, de Wit HM, Stalenhoef AF, de Graaf J (2007) Endothelial function in familial combined hyperlipidaemia. Eur J Clin Invest 37: 381-389

[13] Georgieva AM, Cate HT, Keulen ET, et al. (2004) Prothrombotic markers in familial combined hyperlipidemia: evidence of endothelial cell activation and relation to metabolic syndrome. Atherosclerosis 175: 345-351

[14] Ayyobi AF, Brunzell JD (2003) Lipoprotein distribution in the metabolic syndrome, type 2 diabetes mellitus, and familial combined hyperlipidemia. Am J Cardiol 92: 27-33

[15] Brouwers MC, Bilderbeek-Beckers MA, Georgieva AM, van der Kallen CJ, van Greevenbroek MM, de Bruin TW (2007) Fatty liver is an integral feature of familial combined hyperlipidaemia: relationship with fat distribution and plasma lipids. Clin Sci (Lond) 112: 123-130

[16] Brouwers MC, Van Greevenbroek MM, Bilderbeek-Beckers MA, et al. (2007) Fatty liver based identification of two distinct hypertriglyceridemic subgroups in familial combined hyperlipidemia Metabolism 56: 1311-1317

[17] Farrell GC (2002) Drugs and steatohepatitis. Semin Liver Dis 22: 185-194

[18] Keulen ET, Kruijshoop M, Schaper NC, Hoeks AP, de Bruin TW (2002) Increased intima media thickness in familial combined hyperlipidemia associated with apolipoprotein B. Arterioscler Thromb Vasc Biol 22: 283-288

[19] Saverymuttu SH, Joseph AE, Maxwell JD (1986) Ultrasound scanning in the detection of hepatic fibrosis and steatosis. Br Med J (Clin Res Ed) 292: 13-15

[20] Saadeh S, Younossi ZM, Remer EM, et al. (2002) The utility of radiological imaging in nonalcoholic fatty liver disease. Gastroenterology 123: 745-750

[21] Bekers O, op den Buijsch RA, de Vries JE, Wijnen PA, van Dieijen-Visser MP (2002) Capillary electrophoretic detection in apolipoprotein E genotyping. Electrophoresis 23: 1878-1881

[22] Xu N, Dahlback B, Ohlin AK, Nilsson A (1998) Association of vitamin K-dependent coagulation proteins and $\mathrm{C} 4 \mathrm{~b}$ binding protein with triglyceride-rich lipoproteins of human plasma. Arterioscler Thromb Vasc Biol 18: 33-39

[23] Redgrave TG, Roberts DC, West CE (1975) Separation of plasma lipoproteins by density gradient ultracentrifugation. Anal Biochem 65: 42-49

[24] Sironi L, Mussoni L, Prati L, et al. (1996) Plasminogen activator inhibitor type-1 synthesis and mRNA expression in HepG2 cells are regulated by VLDL. Arterioscler Thromb Vasc Biol 16: 89-96 
[25] Stiko-Rahm A, Wiman B, Hamsten A, Nilsson J (1990) Secretion of plasminogen activator inhibitor-1 from cultured human umbilical vein endothelial cells is induced by very low density lipoprotein. Arteriosclerosis 10: 1067-1073

[26] Schneider WJ, Kovanen PT, Brown MS, et al. (1981) Familial dysbetalipoproteinemia. Abnormal binding of mutant apoprotein $\mathrm{E}$ to low density lipoprotein receptors of human fibroblasts and membranes from liver and adrenal of rats, rabbits, and cows. J Clin Invest 68: 1075-1085

[27] Schalkwijk CG, Stehouwer CD (2005) Vascular complications in diabetes mellitus: the role of endothelial dysfunction. Clin Sci (Lond) 109: 143-159

[28] Targher G, Bertolini L, Scala L, Zoppini G, Zenari L, Falezza G (2005) Non-alcoholic hepatic steatosis and its relation to increased plasma biomarkers of inflammation and endothelial dysfunction in non-diabetic men. Role of visceral adipose tissue. Diabet Med 22: $1354-1358$

[29] Targher G, Bertolini L, Padovani R, Zenari L, Zoppini G, Falezza G (2004) Relation of nonalcoholic hepatic steatosis to early carotid atherosclerosis in healthy men: role of visceral fat accumulation. Diabetes Care 27: 2498-2500

[30] Schindhelm RK, Dekker JM, Nijpels G, et al. (2006) Alanine aminotransferase predicts coronary heart disease events: A 10-year follow-up of the Hoorn Study Atherosclerosis

[31] Ioannou GN, Weiss NS, Boyko EJ, Mozaffarian D, Lee SP (2006) Elevated serum alanine aminotransferase activity and calculated risk of coronary heart disease in the United States Hepatology 43: 1145-1151

[32] Ridker PM, Buring JE, Cook NR, Rifai N (2003) C-reactive protein, the metabolic syndrome, and risk of incident cardiovascular events: an 8-year follow-up of 14719 initially healthy American women. Circulation 107: 391-397

[33] Castell JV, Gomez-Lechon MJ, David M, Hirano T, Kishimoto T, Heinrich PC (1988) Recombinant human interleukin-6 (IL-6/BSF-2/HSF) regulates the synthesis of acute phase proteins in human hepatocytes. FEBS letters 232: 347-350

[34] Alessi MC, Bastelica D, Mavri A, et al. (2003) Plasma PAI-1 Levels Are More Strongly Related to Liver Steatosis Than to Adipose Tissue Accumulation. Arterioscler Thromb Vasc Biol 23: 1262-1268

[35] Yki-Jarvinen H, Sutinen J, Silveira A, et al. (2003) Regulation of plasma PAI-1 concentrations in HAART-associated lipodystrophy during rosiglitazone therapy Arterioscler Thromb Vasc Biol 23: 688-694

[36] Krebs M, Geiger M, Polak K, et al. (2003) Increased plasma levels of plasminogen activator inhibitor-1 and soluble vascular cell adhesion molecule after triacylglycerol infusion in man. Thromb Haemost 90: 422-428

[37] Mermod JJ, Kruithof EK, Alouani S, Quiquerez AL, Sadoul R (1997) ApoE genotype does not affect plasma tPA and PAI-1 antigen levels. Am J Med Genet 74: 172-175

[38] de Beer F, Stalenhoef AF, Hoogerbrugge N, et al. (2002) Expression of type III hyperlipoproteinemia in apolipoprotein E2 (Arg158 --> Cys) homozygotes is associated with hyperinsulinemia. Arterioscler Thromb Vasc Biol 22: 294-299 


\section{Fatty liver in relation to the multiple-type hyperlipidemia}

\subsection{1}

Longitudinal differences in familial combined hyperlipidemia quantitative trait loci

Brouwers MC, Kono N van Greevenbroek MM van der Kallen CJ, Lusis AJ de Bruin TW Cantor RM

Letter to the Editor Arterioscler Thromb Vasc Biol. 2006; 26(6):e118-9 
$106 \quad 2.3 .1$ 
Familial combined hyperlipidemia (FCHL), associated with premature cardiovascular disease, is the most common genetic hyperlipidemia with an estimated prevalence of $1 \%$ [1]. The complex genetic background of FCHL is slowly being dissected through genome screens and positional candidate association studies [2-6]. However, it has also been argued that although numerous studies have been conducted and reported, there are many conflicting results and a true understanding of the genetics remains largely unknown [7]. Previously in this journal, we reported 4 quantitative trait loci (QTL) for apolipoprotein B (apoB), cholesterol, and triglycerides with $\mathrm{p}<0.001$ in 22 FCHL pedigrees from Maastricht, in The Netherlands [8]. The original QTL study was based on the premise that because FCHL is defined by increased serum levels of cholesterol, apoB, and triglycerides in families, QTL analyses of these quantitative traits in FCHL families might identify important genetic loci for this disorder. This letter reports QTL analyses of 5-year follow-up data in the same individuals.

Longitudinal studies show that the lipid phenotype within individual FCHL patients changes over time $[9,10]$. This expected intra-individual variability in cholesterol, triglyceride, and apoB levels may also change the heritabilities of these traits, thus affecting the locations and levels of significance of the reported QTL. However, one might expect that true QTL that are not falsepositives would exhibit consistent chromosome locations, although the levels of significance might vary somewhat. To assess the impact of longitudinal lipid variability on our previous QTL, we recruited all of the Maastricht FCHL family members who participated in the original study and used the same research protocol and QTL analysis strategy used in the original study [8]. The protocol of the current study was approved by the appropriate institutional ethics committees.

Unfortunately, 17 subjects from the original QTL analyses were lost to follow-up. Table 1 shows the characteristics of those who remained in the longitudinal study both at baseline in 1999 and in the follow-up in 2004. Nonparametric Mann-Whitney 2 sample tests found no significant differences in the baseline 1999 body mass index (BMI) and plasma trait values between the whole sample and those 17 lost to follow-up, providing confidence that there was no bias introduced by removing these 17 people from the study.

Table 1.

Baseline and 2004 characteristics

\begin{tabular}{lccc}
\hline & 1999 & 2004 & Variation $\dagger$ \\
\hline Males / females & $24 / 30$ & $24 / 30$ & \\
Age, years & $45.5(28.3-51.3)$ & $49.5(34.0-57.0)$ & \\
BMI, kg/m2 & $26.8(23.6-30.5)$ & $27.7(23.9-30.5)^{*}$ & \\
Apolipoprotein B, g/L & $1.16(0.97-1.4)$ & $1.12(0.97-1.3)$ & $8.3(5.1-17.9)$ \\
Total cholesterol, mmol/L & $5.3(4.7-6.7)$ & $5.3(4.4-6.1)$ & $11.0(5.7-17.7)$ \\
Triglycerides, mmol/L & $1.3(1.0-2.2)$ & $1.6(1.1-3.0)$ & $21.9(8.3-33.6)$ \\
\hline
\end{tabular}

Data are presented as median (interquartile range).

$* \mathrm{p}=0.06$, non-parametric test for related measurements (Wilcoxon). $\dagger$ calculated as $\mathrm{SD}_{1999-2004} /$ mean $_{1999-2004} * 100$ 
However, their exclusion from the 1999 QTL analysis did influence the levels of significance of the original QTL to some extent. The cholesterol QTL on chromosome 12p13 had a marked reduction in the level of significance with their removal ('1999-original' versus '1999';

Figure 1, panel A), making a direct comparison of that QTL from the abbreviated 1999 data with the follow-up 2004 data difficult. The original QTL for the other traits were not affected so markedly by the loss of these 17 subjects (panels B, C, and D), allowing their direct comparison in the location and significance levels with the QTL derived from the 2004 data. The QTL for triglycerides in the abbreviated data set measured in 1999 at 4p15-16 was very similar in shape and location to the one generated after the 5-year follow-up $\left(Z_{\text {max-2004 }}=2.7\right.$ at $40 \mathrm{cM}, \mathrm{p}=0.003$, panel B). The overlap between these longitudinal QTL suggests that the region on 4p15-16 might be narrowed to one between 35 and $45 \mathrm{cM}$ for further studies. There was a greater reduction in the level of significance of the QTL for apoB at 17p11-q21 $\left(Z_{\text {max-2004 }}=1.7\right.$ at $64 \mathrm{cM}, \mathrm{p}=0.04$, panel C). The QTL at 1p21-31 for apoB remained highly significant for the longitudinal data collected in $2004\left(\mathrm{Z}_{\text {max-2004 }}=3.8\right.$ at $137 \mathrm{cM}, \mathrm{p}=0.00007$, panel D), with the QTL having nearly the same shape.

In the early 1980's, Brunzell and colleagues reported that lipid phenotypes in FCHL can change over time, likely because of the interplay of genetic and environmental factors [9] prompting our assessment of the consistency of our QTL. Given the loss to follow-up, 3 of the 4 previously reported QTL [8] were eligible for analysis. We were especially encouraged by the consistency in the location and level of significance of the QTL for apoB at 1p21-31 and the QTL for triglycerides at 4p15-16. The nonparametric QTL analysis applied to these traits may have mitigated the influence of any outliers in the original and follow-up analyses, thus keeping the true QTL consistent.

The present study supports the validity of this QTL approach for gene localization of FCHLrelated quantitative traits over time and prioritizes the 1p21-31 region for fine mapping and positional candidate association studies. 

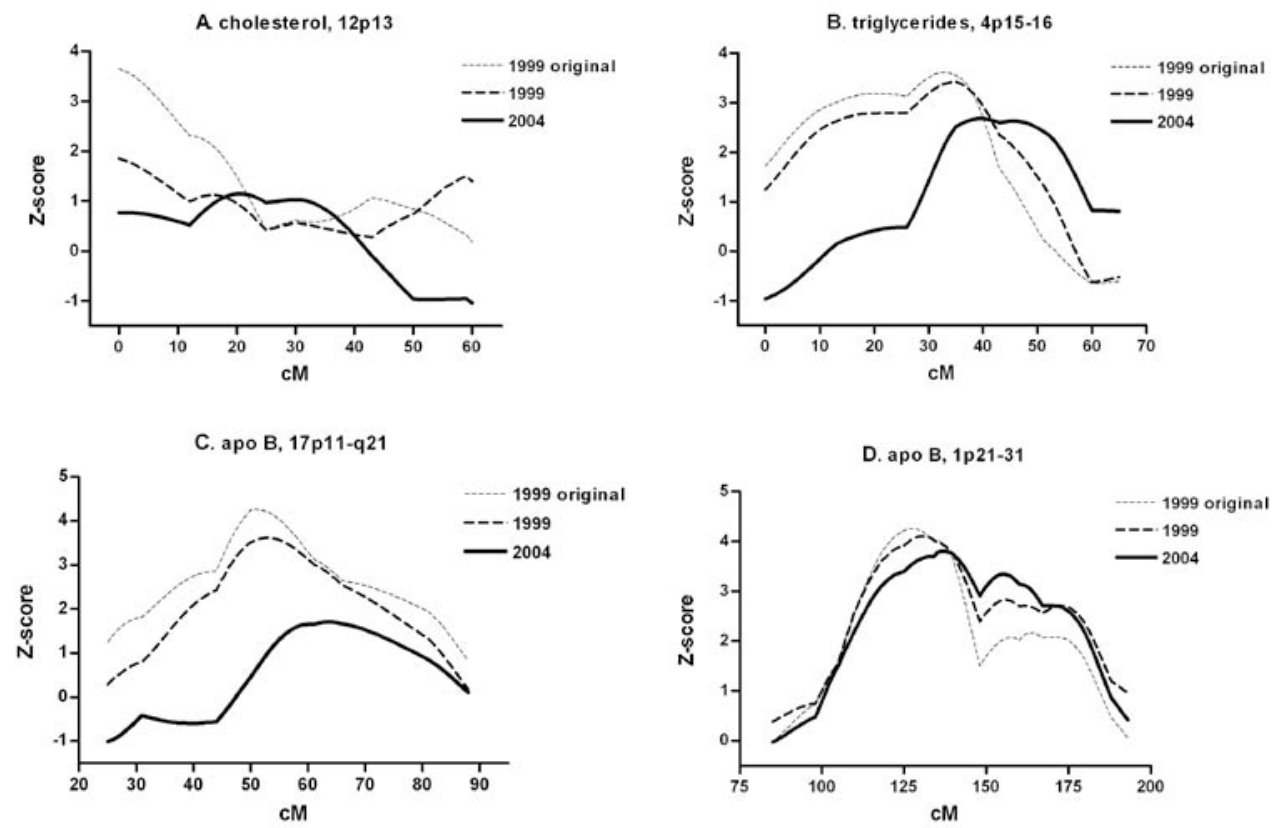

Figure 1.

QTL analyses with 5-year interval for cholesterol (A), triglycerides (B) and apolipoprotein B (C, D). The '1999-original' curves represent the significant QTL as presented in the original report [8]; the '1999' curve is composed of the same measurements as '1999-original', but without the 17 subjects who were lost in follow-up; the '2004' curve is composed of the same population as '1999', but with measurements with 5-years interval.

\section{References}

[1] Goldstein JL, Schrott HG, Hazzard WR, Bierman EL, Motulsky AG (1973) Hyperlipidemia in coronary heart disease. II. Genetic analysis of lipid levels in 176 families and delineation of a new inherited disorder, combined hyperlipidemia. J Clin Invest 52: 1544-1568

[2] Pajukanta P, Lilja HE, Sinsheimer JS, et al. (2004) Familial combined hyperlipidemia is associated with upstream transcription factor 1 (USF1). Nat Genet 36: 371-376

[3] Shoulders CC, Jones EL, Naoumova RP (2004) Genetics of familial combined hyperlipidemia and risk of coronary heart disease. Hum Mol Genet 13 Spec No 1: R149-160

[4] Badzioch MD, Igo RP, Jr., Gagnon F, et al. (2004) Low-density lipoprotein particle size loci in familial combined hyperlipidemia: evidence for multiple loci from a genome scan. 
Arterioscler Thromb Vasc Biol 24: 1942-1950

[5] Naoumova RP, Bonney SA, Eichenbaum-Voline S, et al. (2003) Confirmed Locus on Chromosome 11p and Candidate Loci on $6 \mathrm{q}$ and $8 \mathrm{p}$ for the Triglyceride and Cholesterol Traits of Combined Hyperlipidemia. Arterioscler Thromb Vasc Biol 23: 2070-2077

[6] Naukkarinen J, Gentile M, Soro-Paavonen A, et al. (2005) USF1 and dyslipidemias: converging evidence for a functional intronic variant. Hum Mol Genet 14: 2595-2605

[7] Pollex RL, Hegele RA (2005) Complex trait locus linkage mapping in atherosclerosis: time to take a step back before moving forward? Arterioscler Thromb Vasc Biol 25: $1541-1544$

[8] Cantor RM, de Bruin T, Kono N, et al. (2004) Quantitative trait loci for apolipoprotein $\mathrm{B}$, cholesterol, and triglycerides in familial combined hyperlipidemia pedigrees. Arterioscler Thromb Vasc Biol 24: 1935-1941

[9] Brunzell JD, Albers JJ, Chait A, Grundy SM, Groszek E, McDonald GB (1983) Plasma lipoproteins in familial combined hyperlipidemia and monogenic familial hypertriglyceridemia. J Lipid Res 24: 147-155

[10] Veerkamp MJ, de Graaf J, Bredie SJ, Hendriks JC, Demacker PN, Stalenhoef AF (2002) Diagnosis of familial combined hyperlipidemia based on lipid phenotype expression in 32 families: results of a 5-year follow-up study. Arterioscler Thromb Vasc Biol 22: 274-282 


\section{Fatty liver in relation to the multiple-type hyperlipidemia}

Parabolic relation between plasma triglycerides and LDL-cholesterol: long-term follow-up in the general population, type 2 diabetes mellitus and familial combined hyperlipidemia

Brouwers MC, Georgieva AM van Greevenbroek MM van der Kallen CJ, Heine RJ ter Avest E, de Graaf J Dekker JM, Stalenhoef AF Stehouwer CD de Bruin TW

Published in part in: Clin Sci (Lond). 2007; in press 


\begin{abstract}
To test the hypothesis that plasma LDL-cholesterol and triglycerides levels are related in a parabolic fashion: an increase in triglycerides within the range of plasma triglycerides $0.5-1.5 \mathrm{mmol} /$ $\mathrm{L}$ is associated with an increase in LDL-cholesterol, but further increments in plasma triglycerides $>1.5 \mathrm{mmol} / \mathrm{L}$ are associated with a decrease in LDL-cholesterol.

Data were obtained twice with an interval of six years in 1343 subjects derived from the general population (the Hoorn study). Three groups were defined: one with triglycerides $<1.5 \mathrm{mmol} / \mathrm{L}$ at both time-points (group A), one group with triglycerides $<1.5 \mathrm{mmol} / \mathrm{L}$ at one and $>1.5$ $\mathrm{mmol} / \mathrm{L}$ at the other measurement (group B), and one group with triglycerides $>1.5 \mathrm{mmol} / \mathrm{L}$ at both measurements (group C). In a mixed linear model, a positive relation between triglycerides and LDL-cholesterol was observed for males in group $\mathrm{A}(\beta \mathrm{a}=0.5, \mathrm{p}<0.001)$ and group $\mathrm{B}(\beta \mathrm{b}$ $=0.2, \mathrm{p}<0.001)$, whereas a negative relation was found for males in group $\mathrm{C}(\beta \mathrm{c}=-0.2, \mathrm{p}=$ $0.003)$. The regression slopes did not differ between diabetic and non-diabetic subjects. In the female subgroup, the relation was not significantly negative in group $C(\beta c=-0.1, p=0.4)$. In a second population, consisting of 54 pedigrees with familial combined hyperlipidemia (FCHL, $\mathrm{n}$ $=431$ subjects), a similar longitudinal parabolic relation was observed for men.

The present longitudinal data provide more insight in the complex relation between clinically relevant lipid parameters and show that one cannot interpret one without the other. Furthermore, these results aid to the elucidation of the complex dyslipidemia that characterizes FCHL.
\end{abstract}




\section{Introduction}

Cardiovascular disease is the leading cause of death in western society. One of the major conditional risk factors for cardiovascular disease are elevated low-density lipoprotein (LDL)-cholesterol levels [1-3].

Of interest, subjects with elevated plasma triglycerides levels, such as patients with type 2 diabetes mellitus, often present with fairly normal LDL-cholesterol levels [4, 5]. This, however, does not imply that their cardiovascular risk profile is comparable to the general population. Hypertriglyceridemia has been associated with an increased cardiovascular risk [6], which is for a greater part explained by its clustering with reduced high-density lipoprotein (HDL)-cholesterol levels and the presence of small-dense LDL particles, often referred to as the 'atherogenic lipid triad' [7].

Both triglycerides and cholesterol are transported in plasma by apolipoprotein B containing particles, the former predominantly as very low-density lipoprotein (VLDL)-triglycerides and the latter as LDL-cholesterol. The relationship between (VLDL-) triglycerides and LDL-cholesterol appears to be complex, since previous cross-sectional studies have shown varying results in different populations $[8,9]$.

These contrasting outcomes may be explained by the existence of different subclasses of VLDL, i.e. VLDL 2 and VLDL1, that interact in a different fashion with LDL-particles. The relatively triglyceride-poor VLDL2-particles are the principal VLDL-subclass at low plasma triglycerides levels and are mainly catabolized into cholesterol-rich (buoyant) LDL-particles [10]. The triglyceride-rich VLDL1-particles become abundant when plasma triglycerides levels are elevated [8]. VLDL1-particles exchange triglycerides for cholesterol with LDL-particles resulting in smalldense, relatively cholesterol poor LDL-particles.

We anticipated that the relationship between plasma triglycerides and LDL-cholesterol could be dependent on the actual plasma triglycerides concentration, i.e. at low concentrations, there is a positive relationship, representing the VLDL2-LDL pathway whereas at triglycerides levels above $1.5 \mathrm{mmol} / \mathrm{L}$, when there is increased cholesterol exchange between the triglyceride-rich VLDL-particles and LDL [10], a negative relation can be observed, representing predominance of the VLDL1-small dense LDL pathway.

From the above presented kinetic studies and population based observations we hypothesized that plasma triglycerides and LDL-cholesterol levels are part of a complex metabolic continuum that follows a parabolic relation, as outlined in detail in Figure 1.

In the present study we investigated the hypothesis of a parabolic relationship between fasting plasma triglycerides and LDL-cholesterol in two independent longitudinal studies. In one cohort study, the Hoorn Study [11], 1343 subjects derived from the general population were measured at two different time points with an approximately 6-years interval. In addition, we evaluated the longitudinal observations with the same time interval on plasma triglycerides and LDL-cholesterol in families with a complex genetic dyslipidemia, i.e. familial combined hyperlipidemia (FCHL). 


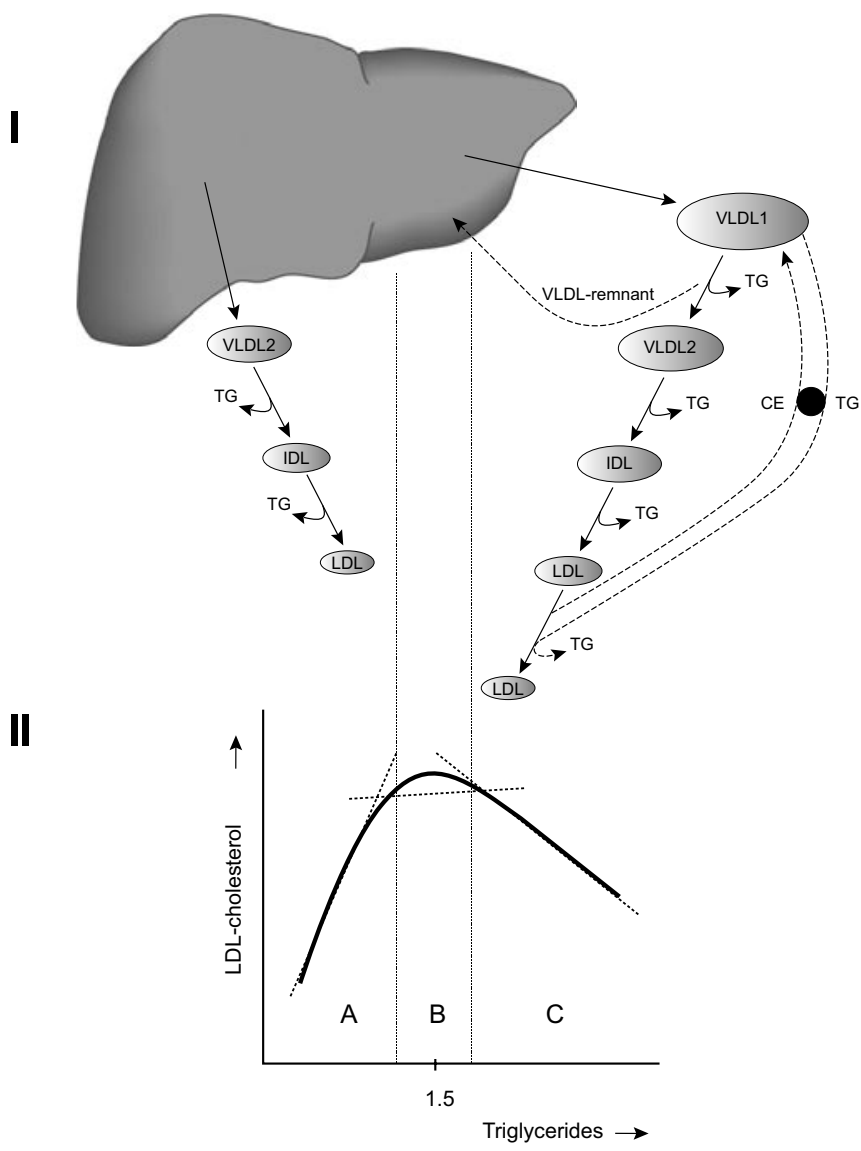

Figure 1.

Hypothesized parabolic relation between plasma triglycerides and LDL-cholesterol. In normotriglyceridemic conditions, triglycerides are mainly incorporated in VLDL2-particles, which are mostly degraded into LDL-particles. Therefore, an increase in VLDL2-particles is expected to lead to an increase in plasma triglycerides and LDL-cholesterol, explaining part A of the parabolic curve in panel II. A further increase in plasma triglycerides is accompanied by an increased secretion of triglyceride-rich VLDL1-particles. VLDL1-particles exchange cholesteryl-ester and triglycerides with LDL- (and HDL-) particles, mediated by cholesteryl-ester transfer protein (CETP), resulting in cholesterol depleted LDL-particles. Many cholesteryl-esters transferred to VLDL1-particles leave the plasma cholesterol pool, since it is estimated that a large proportion of the VLDL1-particles is not catabolized to VLDL2 and eventually to LDL, but is directly taken up as VLDL-remnants by, among others, the liver. This would explain the negative correlation between plasma triglycerides and LDL-cholesterol (part C of the parabolic curve in panel II). Part B of the parabolic curve in panel II depicts the transition from VLDL2-secretion to VLDL1-secretion by the liver. This occurs, on average, at plasma triglycerides $1.5 \mathrm{mmol} / \mathrm{L}$. The three dashed lines in panel II indicate the three subgroups that have been constructed to test our hypothesis of a parabolic relation, see materials and methods sections. 


\section{Materials and methods}

\section{Study population}

General population, the Hoorn Study. Subjects were participants of the Hoorn study, a prospective-based cohort study on glucose intolerance in a random sample of 50 to 75-year old individuals taken from the population register of the town Hoorn in the Netherlands [11, 12]. As described previously [13], of all 2484 subjects who initially participated in baseline examinations (1989-1992), 971 (39\%) did not participate in the 1996-1998 follow-up investigation, for several reasons. Another 151 subjects were excluded because of missing values $(n=36)$ or use of lipid lowering medication $(\mathrm{n}=115)$.

FCHL families, Nijmegen and Maastricht cohort. FCHL was originally described as the presence of different hyperlipidemic phenotypes, i.e. hypertriglyceridemia, hypercholesterolemia or both, within one family [14]. FCHL family members were collected and followed prospectively in two cohorts that were analyzed together: the Nijmegen and Maastricht cohort. The Nijmegen FCHL families ( $n=299$ subjects) were originally recruited based on the 90th percentile diagnostic criteria for hypercholesterolemia and hypertriglyceridemia, which has been described in detail elsewhere, together with their baseline (1999) and follow-up (2004) characteristics [15]. Baseline (1999) criteria and descriptives of the Maastricht FCHL population have also been described previously [16]. Of the 195 subjects who initially participated in the Maastricht study, 50 were lost in follow-up (death $\mathrm{n}=8$, reluctance to participate $\mathrm{n}=42$ )

Baseline characteristics of both cohorts were very similar (Table 1). In both cohorts, type 2 diabetes mellitus was an exclusion criterion for the index patient $[15,16]$. No subjects carried the apo E2/E2 genotype. Furthermore, FCHL patients were withdrawn from lipid lowering medication for at least two weeks prior to both visits. The baseline hyperlipidemic (affected) state was defined according to the recently proposed criteria: fasting triglycerides $>1.5 \mathrm{mmol} / \mathrm{L}$ and apolipoprotein $\mathrm{B}>1.2 \mathrm{~g} / \mathrm{L}$ [17], which is based on previous empirical evidence [18].

The studies were approved by the ethics committee of the University Hospital Vrije Universiteit Amsterdam, the University Medical Center Nijmegen and the Academic Hospital Maastricht/ Maastricht University. All subjects gave written informed consent.

Table 1.

Baseline comparison between Maastricht and Nijmegen affected and non-affected FCHL family members

\begin{tabular}{lcccc}
\hline & \multicolumn{2}{c}{ Non-affected } & \multicolumn{2}{c}{ Affected } \\
& Maastricht & Nijmegen & Maastricht & Nijmegen \\
\hline Male / female, n & $41 / 64$ & $89 / 119$ & $16 / 18$ & $43 / 41$ \\
Age, years & $41.5 \pm 16.3$ & $35.2 \pm 15.5^{*}$ & $46.8 \pm 12.9$ & $49.4 \pm 12.9$ \\
Total cholesterol, mmol/L & $5.2 \pm 1.1$ & $5.2 \pm 1.1$ & $6.7 \pm 1.0$ & $6.9 \pm 0.9$ \\
Triglycerides, mmol/L & $1.3(0.9-1.4)$ & $1.0(0.8-1.3)$ & $2.0(1.7-2.5)$ & $2.1(1.8-2.8)$ \\
Apolipoprotein B, g/L & $1.0 \pm 0.2$ & $0.9 \pm 0.2$ & $1.5 \pm 0.2$ & $1.4 \pm 0.2$ \\
\hline
\end{tabular}

Data are presented as mean $\pm \mathrm{SD}$ or as median (interquartile range); ${ }^{*} \mathrm{p}=0.001$ non-affected relatives Maastricht versus Nijmegen, Student's test; All analyses between Nijmegen and Maastricht were age adjusted. 


\section{Measurements}

Subjects visited the study centers after an overnight fast. Weight and height were measured with participants wearing light clothing only, and the body mass index (BMI) was calculated as weight divided by height squared $\left(\mathrm{kg} / \mathrm{m}^{2}\right)$.

Fasting plasma triglycerides, total cholesterol, HDL-cholesterol and apolipoprotein B were determined as described elsewhere (the latter variable was measured in FCHL only) [15, 16, 19]. LDL-cholesterol was calculated with the Friedewald formula (LDL-cholesterol $=$ total cholesterol - HDL-cholesterol - (plasma triglycerides * 0.45) [20]. Since this formula is only valid for plasma triglycerides $<4.5 \mathrm{mmol} / \mathrm{L}$ [20], subjects with triglycerides above this value were excluded from analyses $(n=19$ in the general population and $n=13$ in FCHL families). In the Nijmegen FCHL cohort, LDL-cholesterol was also approximated by subtracting VLDLcholesterol and HDL-cholesterol from total cholesterol levels. VLDL-cholesterol was measured by ultracentrifugation [18]. All subjects from the general population (Hoorn study) underwent a 75-g oral glucose tolerance test (OGTT) as described previously [11]. Diabetes mellitus was diagnosed according to the WHO-1999 criteria [21].

\section{Statistical analyses}

Baseline characteristics of the cohorts are presented as mean (SD) or median (interquartile ranges). Since sex hormones are known to influence lipid metabolism [22], all analyses were conducted for male and female subjects separately. A Student's T-test was used to test for differences between males and females, and between affected and non-affected FCHL family members. Because of non-normal distribution, plasma triglycerides were log-transformed.

To test the possibility of a parabolic relation between plasma concentrations of triglycerides and LDL-cholesterol, three groups were defined (Figure 1): a group with plasma triglycerides $<1.5$ $\mathrm{mmol} / \mathrm{L}$ both at baseline and at follow-up (group A), a group with triglycerides $>1.5 \mathrm{mmol} / \mathrm{L}$ at both time points (group C), and the remaining group in whom plasma triglycerides were $<1.5$ $\mathrm{mmol} / \mathrm{L}$ at one time-point and $>1.5 \mathrm{mmol} / \mathrm{L}$ at the other moment (group B). The cut-off value of $1.5 \mathrm{mmol} / \mathrm{L}$ was used since it is commonly accepted that from this value there is substantial cholesteryl ester exchange between VLDL1 and LDL-particles (Figure 1) [10]. A mixed linear model for repeated measurements with random intercept was used accounting for repeated measurement in one individual. A significant relation should therefore be interpreted as the intraindividual relation averaged for all subjects in the particular subgroup. In the FCHL population, cohort (Nijmegen/Maastricht) was entered as a fixed variable. According to our hypothesis, we anticipated a significant, positive relation in group A, a significant, negative relation in group C and a relation intermediate to group $\mathrm{A}$ and $\mathrm{C}$ in group $\mathrm{B}$ (Figure 1). In addition, the regression slopes of the different subgroups were compared with each other by using interaction terms. All analyses were conducted with SPSS 13.0 statistical package (SPSS Inc. Illinois, USA). 


\section{Results}

Longitudinal relation between plasma triglycerides and LDL-cholesterol in the general population

Baseline characteristics of male $(n=616)$ and female $(n=727)$ subjects, derived from the general population (Hoorn study), are presented in Table 2. Male subjects were less obese, had higher plasma triglycerides and significantly lower total cholesterol, LDL-cholesterol and HDLcholesterol compared to the female subjects.

As shown in Figure 2 panel I, mixed linear regression analysis revealed a 6 -year positive relation between plasma triglycerides and LDL-cholesterol for males in group A (plasma triglycerides below $1.5 \mathrm{mmol} / \mathrm{L}$ at both time points) and group B (plasma triglycerides below and above 1.5 $\mathrm{mmol} / \mathrm{L}$ at different time points). This relation was also significant, but negative for males in group C (plasma triglycerides above $1.5 \mathrm{mmol} / \mathrm{L}$ at both time points). According to our hypothesis, the slopes of the regression lines, indicated as $\beta$, decreased with increasing plasma triglycerides (Figure 2, panel I). These slopes were all significantly different from each other ( $\beta$ a versus $\beta b: p=0.02 ; \beta b$ versus $\beta c: p<0.001 ; \beta a$ versus $\beta c: p<0.001$, Hochberg corrected).

Of interest, when the longitudinal relation was studied in male subjects who were diabetic at the first and/or second visit ( $\mathrm{n}=39,31$ and 40 in group A, B and C, respectively), a very similar relation could be discerned (Figure 2, panel I, inset). For each subgroup, the regression slopes were not significantly different between diabetic and non-diabetic subjects (data not shown).

In the female population, the regression slopes of the 6-year longitudinal relation between plasma triglycerides and LDL-cholesterol also decreased from group A, to group B, to group C, but the negative relation in the latter group did not reach significance (Figure 2, panel II). All slopes were significantly different from each other $(\beta a$ versus $\beta b: p=0.003 ; \beta b$ versus $\beta c: p=0.02 ; \beta a$ versus $\beta \mathrm{c}: \mathrm{p}<0.001$, Hochberg corrected). Analogous to the male population, a similar relation was observed in the female diabetic subjects ( $\mathrm{n}=36,33$ and 51 in group $A, B$ and $C$ respectively; Figure 2, panel II, inset).

Table 2.

Baseline Characteristics of Hoorn Population

\begin{tabular}{lcc}
\hline & Men $(\mathrm{n}=616)$ & Women $(\mathrm{n}=727)$ \\
\hline Age, years & $60.5 \pm 7.0$ & $59.9 \pm 7.0$ \\
Body mass index, $\mathrm{kg} / \mathrm{m} 2$ & $25.9 \pm 2.8$ & $26.2 \pm 3.6^{*}$ \\
Triglycerides, $\mathrm{mmol} / \mathrm{L}$ & $1.4(1.0-1.8)$ & $1.2(0.9-1.6)^{*}$ \\
Total cholesterol, mmol/L & $6.3 \pm 1.0$ & $6.7 \pm 1.1^{*}$ \\
HDL-cholesterol, mmol/L & $1.2 \pm 0.3$ & $1.5 \pm 0.4^{*}$ \\
LDL-cholesterol, mmol/L & $4.4 \pm 0.9$ & $4.6 \pm 1.0^{*}$ \\
\hline
\end{tabular}

Data are presented as mean $\pm \mathrm{SD}$ or as median (interquartile range);

$* \mathrm{p}<0.01$ males versus females, Student's T-test 

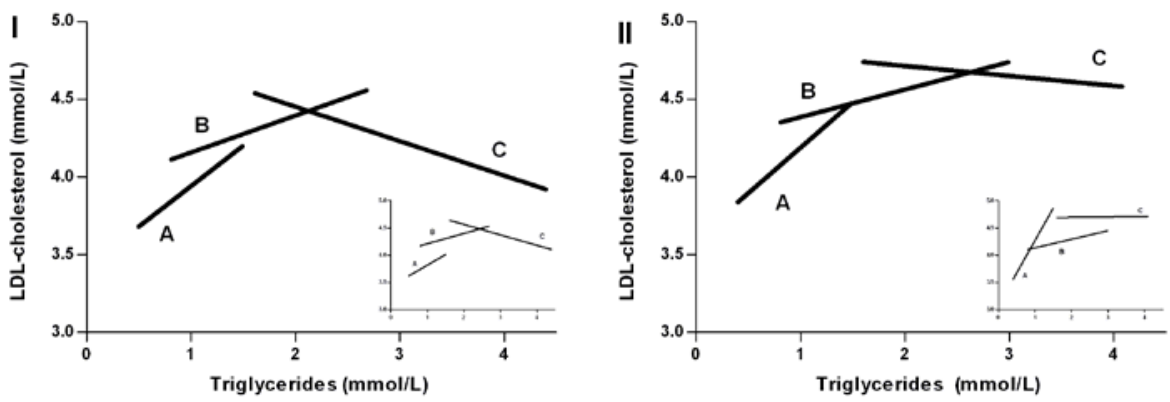

Figure 2.

6-year intra-individual relation between plasma triglycerides and LDL-cholesterol averaged for male (panel I) and female (panel II) subgroups, derived from the general population (Men: group A: $\mathrm{n}=303, \beta \mathrm{a}=0.5, \mathrm{p}<0.001$; group B: $\mathrm{n}=167, \beta \mathrm{b}=0.2, \mathrm{p}<0.001$; group $\mathrm{C}: \mathrm{n}=146, \beta \mathrm{c}=-0.2, \mathrm{p}=0.003$. Women: group $\mathrm{A}: \mathrm{n}=407, \beta \mathrm{a}=0.6, \mathrm{p}<0.001$; group $B: n=163, \beta b=0.2, p=004$; group $C: n=157, \beta c=-0.1, p=0.4$ ).

The insets show the same relation, but for diabetic subjects only. Regression lines are drawn from the minimum to the maximum triglycerides value of each subgroup.

\section{Longitudinal relation between plasma triglycerides and LDL-cholesterol in FCHL families}

We additionally studied the longitudinal relation between plasma triglycerides and LDL-cholesterol in two cohorts of FCHL families that were combined in this analysis. Table 3 summarizes the baseline characteristics of the 54 FCHL families, split by gender and the affected state. Since the affected state depends on plasma triglycerides (cut-off $1.5 \mathrm{mmol} / \mathrm{L}$ ) [17], subsequent analyses were conducted in all FCHL family members, i.e. affected and non-affected relatives combined, to obtain a population with plasma triglycerides ranging from normotriglyceridemia to hypertriglyceridemia, which enabled us to study the possible presence of a parabolic relation. Comparable to what as described above, a 5-year longitudinal parabolic relation between plasma triglycerides and LDL-cholesterol was present in the male FCHL family members, as reflected by a significant, positive relation in group $\mathrm{A}(\beta \mathrm{a}=1.1, \mathrm{p}<0.001)$, a significant, negative relation in group $C(\beta c=-0.3, p=0.002)$ and a relation intermediate to group $A$ and group $C$ in group $B$ $(\beta b=0.2, p=0.1)$, as shown in Figure 3, panel I. All regression slopes were significantly different from each other ( $\beta$ a versus $\beta b: p=0.001 ; \beta b$ versus $\beta$ : $p<0.001 ; \beta$ a versus $\beta c: p<0.001$, Hochberg corrected). Of note, sub-analysis in the Nijmegen cohort revealed very similar results when LDL-cholesterol levels, approximated by subtracting VLDL-cholesterol and HDL-cholesterol from total cholesterol levels, were taken for analysis (Figure 3, panel I, inset). Therefore, the use of the Friedewald formula does not account for the observed parabola.

Interestingly, substituting LDL-cholesterol by apolipoprotein B levels, representing the total amount of atherogenic particles in plasma, did not yield a parabolic model in the overall male FCHL population (Figure 3, panel II).

A five-year longitudinal relation between plasma triglycerides and LDL-cholesterol was not found in any of the female FCHL subgroups (group A: $n=132, \beta a=0.1, p=0.6$; group B: $n=$ 
$45, \beta b=-0.01, p=0.9$; group $C: n=64, \beta c=-0.1, p=0.5)$, nor was a relation observed between plasma triglycerides and apolipoprotein $\mathrm{B}$.

Table 2.

Baseline characteristics of FCHL population

\begin{tabular}{lcccc}
\hline & \multicolumn{2}{c}{ Men } & \multicolumn{2}{c}{ Women } \\
& $\begin{array}{c}\text { Non-affected } \\
(\mathrm{n}=131)\end{array}$ & $\begin{array}{c}\text { Affected } \\
(\mathrm{n}=59)\end{array}$ & $\begin{array}{c}\text { Non-affected } \\
(\mathrm{n}=182)\end{array}$ & $\begin{array}{c}\text { Affected } \\
(\mathrm{n}=59)\end{array}$ \\
\hline Age, years & $37.1 \pm 15.9$ & $46.7 \pm 11.6^{*}$ & $37.4 \pm 16.2$ & $50.7 \pm 13.9^{*}$ \\
Body mass index, $\mathrm{kg} / \mathrm{m}^{2}$ & $23.9 \pm 3.5$ & $26.4 \pm 3.1^{*}$ & $24.0 \pm 3.9$ & $27.4 \pm 4.2^{*}$ \\
Triglycerides, $\mathrm{mmol} / \mathrm{L}$ & $1.1(0.9-1.6)$ & $2.0(1.8-2.6)^{*}$ & $1.0(0.8-1.3) \dagger$ & $2.1\left(1.8-2.8^{*}\right.$ \\
Total cholesterol, $\mathrm{mmol} / \mathrm{L}$ & $5.1 \pm 1.1$ & $6.8 \pm 1.0^{*}$ & $5.2 \pm 1.1$ & $7.0 \pm 1.0^{*}$ \\
HDL-cholesterol, $\mathrm{mmol} / \mathrm{L}$ & $1.1 \pm 0.3$ & $0.9 \pm 0.2^{*}$ & $1.3 \pm 0.3 \dagger$ & $1.0 \pm 0.3^{*} \dagger$ \\
LDL-cholesterol, mmol/L & $3.5 \pm 1.1$ & $4.9 \pm 0.8^{*}$ & $3.5 \pm 1.0$ & $4.9 \pm 1.0^{*}$ \\
Apolipoprotein $\mathrm{B}, \mathrm{g} / \mathrm{L}$ & $1.0 \pm 0.2$ & $1.4 \pm 0.2^{*}$ & $1.0 \pm 0.2$ & $1.5 \pm 0.2^{*}$ \\
\hline
\end{tabular}

Data are presented as mean \pm SD or as median (interquartile range); the affected state was defined as apolipoprotein $\mathrm{B}>1.2 \mathrm{~g} / \mathrm{L}$ and triglycerides $>1.5 \mathrm{mmol} / \mathrm{L}$.

${ }^{*} \mathrm{p}<0.001$ non-affected versus affected FCHL family members with the same gender, Student's T-test t $\mathrm{p}<0.05$ men versus women with the same lipid status, Student's T-test
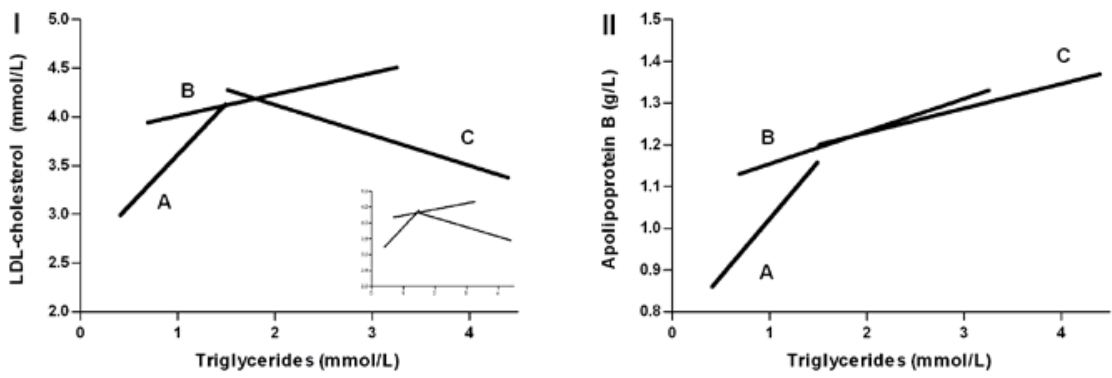

Figure 3.

5-year intra-individual relation between plasma triglycerides and LDL-cholesterol (panel I) and apolipoprotein B levels (panel II) averaged for male FCHL subgroups (LDL-cholesterol: group A: $\mathrm{n}=65, \beta \mathrm{a}=1.1, \mathrm{p}<0.001$; group $B: n=54, \beta b=0.2, p=0.1$; group $C: n=71, \beta c=-0.3, p=0.002$. Apolipoprotein B: group A: $\beta a=0.3, p<0.001$; group $B \beta b=0.1, p=0.02$; group $C: \beta c=0.1, p=0.004)$. The inset of panel I shows the results for LDL-cholesterol approximated by subtracting VLDL-cholesterol and HDL-cholesterol from total cholesterol in the Nijmegen FCHL population. Regression lines are drawn from the minimum to the maximum triglycerides value of each subgroup. 


\section{Intra-individual parabolic relation between plasma triglycerides and LDL-cholesterol}

Further evidence for the above presented longitudinal relation between plasma triglycerides and LDL-cholesterol was explored by investigating whether one individual can pass completely trough the parabolic curve. Therefore, at least three consecutive measurements in one individual were required with plasma triglycerides values below, around and above the parabolic peak value of $1.5 \mathrm{mmol} / \mathrm{L}$. Although such series of data are hard to obtain for each individual, 13 male subjects in the FCHL population were measured more than twice and fulfilled each of the following plasma triglycerides criteria: one measurement around $1.5 \mathrm{mmol} / \mathrm{L}$, i.e. between the arbitrary values of 1.4 and $1.6 \mathrm{mmol} / \mathrm{L}$, one measurement below $1.4 \mathrm{mmol} / \mathrm{L}$ and one measurement above $1.6 \mathrm{mmol} / \mathrm{L}$. Of the 13 subjects who met these criteria, 11 subjects showed an increase in LDL-cholesterol levels when plasma triglycerides values increased from low to moderate levels $(\mathrm{p}=0.02)$, whereas a further increase was accompanied with a decrease in LDL-cholesterol ( $p$ $=0.006$ ) (Figure 4).
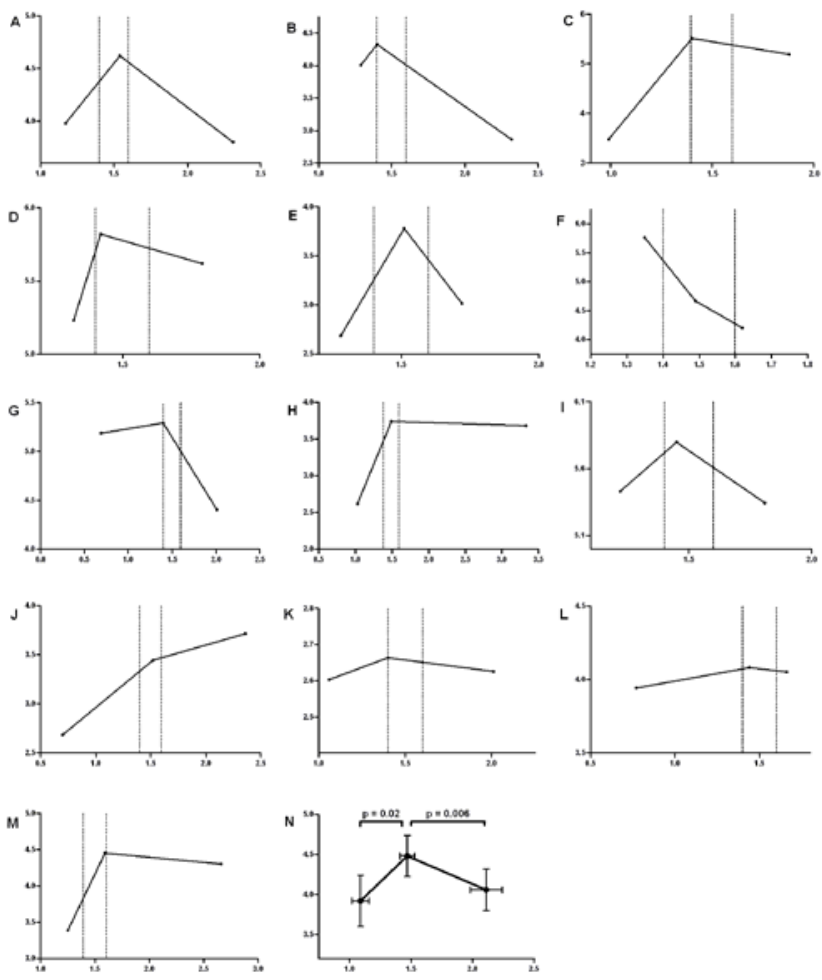

Figure 4.

Intra-individual relation between plasma triglycerides and LDL-cholesterol for 13 FCHL family members with each three consecutive measurements below triglycerides $1.4 \mathrm{mmol} / \mathrm{L}$, between 1.4 and $1.6 \mathrm{mmol} / \mathrm{L}$ and above $1.6 \mathrm{mmol} / \mathrm{L}$ (Panel A to M). Dashed vertical lines indicate plasma triglycerides $1.4 \mathrm{mmol} / \mathrm{L}$ and $1.6 \mathrm{mmol} / \mathrm{L}$. Panel $\mathrm{N}$ depicts the average relation. Error bars represent SEM. Analyzed with Wilcoxon's non-parametric test for paired samples. 


\section{Discussion}

The present longitudinal study was conducted in two study populations with a follow-up duration of five and six years to test the hypothesis that a longitudinal parabolic relation exists between triglycerides and cholesterol. Such a relation could explain some of the complex and seemingly contradicting data that are present in the literature. The data revealed that the longitudinal relation between plasma triglycerides and LDL-cholesterol indeed follows a parabolic curvature in male subjects, not only in the general population, but also in the two most common forms of dyslipidemia, i.e. type 2 diabetes mellitus and familial combined hyperlipidemia (FCHL). The hypothesized relation was based on the fact that triglycerides can be incorporated into either VLDL1 - or VLDL2-particles that are known to have distinct catabolic pathways (figure 1) [10].

The verification of our hypothesized parabola required dividing the general (Hoorn) population and the FCHL families into three subgroups. This approach revealed a significant positive intra-individual relation between plasma triglycerides and LDL-cholesterol for male subjects with plasma triglycerides below $1.5 \mathrm{mmol} / \mathrm{L}$ and a significant negative relation for male subjects with plasma triglycerides above this threshold, which is suggestive of an intra-individual parabolic relation between plasma triglycerides and LDL-cholesterol. Of interest, 11 of 13 male FCHL family members with observations in each of the different triglycerides segments individually passed through the complete parabolic curve, substantiating the evidence for the parabolic relation between triglycerides and LDL cholesterol.

Interventional studies aimed at lowering plasma triglycerideds levels are very much in concordance with our epidemiological data. Ginsberg et al. reported a significant reduction of plasma triglycerides after weight loss in hypertriglyceridemic subjects, which was accompanied by an increase in LDL-cholesterol levels due to a decreased clearance and to a beneficial shift from small-dense LDL-particles towards more buoyant particles [23]. Very similar results have been reported for therapy with fibrates [24], one of the second-line therapeutic modalities to reduce triglycerides levels [25].

Therefore, the observed parabola stresses the necessity to take into account plasma triglycerides concentrations when LDL-cholesterol levels are monitored over time, e.g. during intervention. An increase in plasma triglycerides is accompanied by an increase in LDL-cholesterol that subsequently falls when plasma triglycerides rise further. Of importance, we do not suggest that a parabolic relation between plasma triglycerides and LDL-cholesterol is synonymous to a parabolic relation between plasma triglycerides and cardiovascular risk. Elevated plasma triglycerides cluster with reduced HDL-cholesterol levels and the presence of small-dense LDL-particles into the atherogenic lipid triad [7] that confers an increased risk of cardiovascular disease [26, 27]. The decrease in the LDL-cholesterol/apolipoprotein $B$ ratio that coincided with the rise in plasma triglycerides is indeed indicative of small-dense LDL. Therefore, the present, almost linear, longitudinal relation between plasma triglycerides and apolipoprotein B in the male FCHL population seems to corroborate the suggestion that apolipoprotein B levels are a better marker 
for cardiovascular risk estimation than LDL-cholesterol [28], in particular in hypertriglyceridemic conditions.

The currently observed parabolic relation between plasma triglycerides and LDL-cholesterol is in line with the observation that many hypertriglyceridemic subjects, including patients with type 2 diabetes mellitus, frequently display normal LDL-cholesterol levels $[4,5]$. In addition, it provides more insight in the complex 'multiple-type hyperlipidemia' that characterizes FCHL. This highly prevalent genetic hyperlipidemia was originally described as the presence of different lipid phenotypes, i.e. hypercholesterolemia, hypertriglyceridemia or the combination of both, within one family [14]. Almost one decade after the first description, Brunzell and colleagues reported that these different lipid phenotypes can also occur within one FCHL individual over time, which was later confirmed by others $[9,15]$. The current data suggest that this so-called 'multiple-type hyperlipidemia', a phenomenon that complicates the diagnosis and elucidation of FCHL, may not be as heterogeneous as it appears at first sight: as plasma triglycerides increase, an individual FCHL patient will progress through the parabolic curvature and will consecutively present with isolated hypercholesterolemia, combined hyperlipidemia (both hyperhypercholesterolemia and hypertriglyceridemia) and eventually with isolated hypertriglyceridemia, accounting for the different lipid phenotypes over time. Since the parabolic curvature is valid not only for FCHL, but also for the general population and type 2 diabetes mellitus, the current study suggests that the 'multiple-type hyperlipidemia' can occur in all individuals. However, the 'multiple-type hyperlipidemia' has always been associated specifically to FCHL, which might be explained by the fact that FCHL individuals are more likely to balance around the cut-off values for hypercholesterolemia and hypertriglyceridemia due to an increased genetic susceptibility to elevated baseline cholesterol and triglycerides levels [29, 30].

In the present study, a parabolic relation was observed in men, but not in women. Estrogens exert a well-known effect on lipid metabolism [22], among others by affecting hepatic lipase activity [31]. It is expected that the composition of the female FCHL population, i.e. pre-menopausal and postmenopausal women, in combination with the varying estrogens levels over time in the pre-menopausal women may have introduced a substantial noise in the dataset. In contrast, the female subset of the general population appeared to be more homogeneous, since the baseline age of the female subjects was on average $59.9 \pm 7.0$ years, which is far above the average age of onset of the menopause [32]. This may explain the presence of a significant, longitudinal relation in this group, although it was not identical to men. This may be accounted for by the fact that, despite the absence of estrogens in postmenopausal women, the activity of hepatic lipase, a key enzyme in the formation small-dense LDL-particles [10], is still lower compared to men [33].

In summary, the present follow-up study in two independent populations has provided epidemiological data that, within an individual, plasma triglycerides and LDL-cholesterol levels are related in a parabolic fashion over time. The complex relation between these two clinically relevant parameters shows that one cannot be interpreted without the other, which is relevant in case 
of pharmacological interventions. In addition, it might aid to the elucidation of the complex dyslipidemia that characterizes FCHL.

\section{References}

[1] Kannel WB, Dawber TR, Friedman GD, Glennon WE, McNamara PM (1964) Risk Factors in Coronary Heart Disease. An Evaluation of Several Serum Lipids as Predictors of Coronary Heart Disease; the Framingham Study. Ann Intern Med 61: 888-899

[2] Pekkanen J, Linn S, Heiss G, et al. (1990) Ten-year mortality from cardiovascular disease in relation to cholesterol level among men with and without preexisting cardiovascular disease. N Engl J Med 322: 1700-1707

[3] Stamler J, Daviglus ML, Garside DB, Dyer AR, Greenland P, Neaton JD (2000) Relationship of baseline serum cholesterol levels in 3 large cohorts of younger men to long-term coronary, cardiovascular, and all-cause mortality and to longevity. Jama 284: $311-318$

[4] Ayyobi AF, Brunzell JD (2003) Lipoprotein distribution in the metabolic syndrome, type 2 diabetes mellitus, and familial combined hyperlipidemia. Am J Cardiol 92: 27-33

[5] Sacks FM, Tonkin AM, Craven T, et al. (2002) Coronary heart disease in patients with low LDL-cholesterol: benefit of pravastatin in diabetics and enhanced role for HDLcholesterol and triglycerides as risk factors. Circulation 105: 1424-1428

[6] Austin MA, Hokanson JE, Edwards KL (1998) Hypertriglyceridemia as a cardiovascular risk factor. Am J Cardiol 81: 7B-12B

[7] Austin MA, King MC, Vranizan KM, Krauss RM (1990) Atherogenic lipoprotein phenotype. A proposed genetic marker for coronary heart disease risk. Circulation 82: 495-506

[8] Tan CE, Foster L, Caslake MJ, et al. (1995) Relations between plasma lipids and postheparin plasma lipases and VLDL and LDL subfraction patterns in normolipemic men and women. Arterioscler Thromb Vasc Biol 15: 1839-1848

[9] Brunzell JD, Albers JJ, Chait A, Grundy SM, Groszek E, McDonald GB (1983) Plasma lipoproteins in familial combined hyperlipidemia and monogenic familial hypertriglyceridemia. J Lipid Res 24: 147-155

[10] Packard CJ, Shepherd J (1997) Lipoprotein heterogeneity and apolipoprotein B metabolism. Arterioscler Thromb Vasc Biol 17: 3542-3556

[11] Mooy JM, Grootenhuis PA, de Vries H, et al. (1995) Prevalence and determinants of glucose intolerance in a Dutch caucasian population. The Hoorn Study. Diabetes Care 18: $1270-1273$

[12] Girman CJ, Dekker JM, Rhodes T, et al. (2005) An exploratory analysis of criteria for the metabolic syndrome and its prediction of long-term cardiovascular outcomes: the Hoorn study. Am J Epidemiol 162: 438-447

[13] de Vegt F, Dekker JM, Jager A, et al. (2001) Relation of impaired fasting and postload glucose with incident type 2 diabetes in a Dutch population: The Hoorn Study. Jama 285: 2109-2113 
[14] Goldstein JL, Schrott HG, Hazzard WR, Bierman EL, Motulsky AG (1973)

Hyperlipidemia in coronary heart disease. II. Genetic analysis of lipid levels in 176 families and delineation of a new inherited disorder, combined hyperlipidemia. J Clin Invest 52: 1544-1568

[15] Veerkamp MJ, de Graaf J, Bredie SJ, Hendriks JC, Demacker PN, Stalenhoef AF (2002) Diagnosis of familial combined hyperlipidemia based on lipid phenotype expression in 32 families: results of a 5-year follow-up study. Arterioscler Thromb Vasc Biol 22: 274-282

[16] Keulen ET, Voors-Pette C, de Bruin TW (2001) Familial dyslipidemic hypertension syndrome: familial combined hyperlipidemia, and the role of abdominal fat mass. Am J Hypertens 14: 357-363

[17] Sniderman AD, Castro Cabezas M, Ribalta J, et al. (2002) A proposal to redefine familial combined hyperlipidaemia -- third workshop on FCHL held in Barcelona from 3 to 5 May 2001, during the scientific sessions of the European Society for Clinical Investigation. Eur J Clin Invest 32: 71-73

[18] Veerkamp MJ, de Graaf J, Hendriks JC, Demacker PN, Stalenhoef AF (2004) Nomogram to diagnose familial combined hyperlipidemia on the basis of results of a 5-year followup study. Circulation 109: 2980-2985

[19] Bos G, Dekker JM, Nijpels G, et al. (2003) A combination of high concentrations of serum triglyceride and non-high-density-lipoprotein-cholesterol is a risk factor for cardiovascular disease in subjects with abnormal glucose metabolism--The Hoorn Study. Diabetologia 46: 910-916

[20] Friedewald WT, Levy RI, Fredrickson DS (1972) Estimation of the concentration of lowdensity lipoprotein cholesterol in plasma, without use of the preparative ultracentrifuge. Clin Chem 18: 499-502

[21] (1999) Definition, diagnosis and classification of Diabetes Mellitus and its complications. Report of a WHO consultation. Part 1: Diagnosis and classification of diabetes mellitus. In, Geneva

[22] Campos H, Walsh BW, Judge H, Sacks FM (1997) Effect of estrogen on very low density lipoprotein and low density lipoprotein subclass metabolism in postmenopausal women. J Clin Endocrinol Metab 82: 3955-3963

[23] Ginsberg HN, Le NA, Gibson JC (1985) Regulation of the production and catabolism of plasma low density lipoproteins in hypertriglyceridemic subjects. Effect of weight loss. J Clin Invest 75: 614-623

[24] Vega GL, Grundy SM (1985) Gemfibrozil therapy in primary hypertriglyceridemia associated with coronary heart disease. Effects on metabolism of low-density lipoproteins. Jama 253: 2398-2403

[25] (2002) Third Report of the National Cholesterol Education Program (NCEP) Expert Panel on Detection, Evaluation, and Treatment of High Blood Cholesterol in Adults (Adult Treatment Panel III) final report. Circulation 106: 3143-3421

[26] Austin MA, Breslow JL, Hennekens CH, Buring JE, Willett WC, Krauss RM (1988) Lowdensity lipoprotein subclass patterns and risk of myocardial infarction. Jama 260: 1917-1921 
[27] Lamarche B, Tchernof A, Moorjani S, et al. (1997) Small, dense low-density lipoprotein particles as a predictor of the risk of ischemic heart disease in men. Prospective results from the Quebec Cardiovascular Study. Circulation 95: 69-75

[28] Barter PJ, Ballantyne CM, Carmena R, et al. (2006) Apo B versus cholesterol in estimating cardiovascular risk and in guiding therapy: report of the thirty-person/ten-country panel. J Intern Med 259: 247-258

[29] van der Kallen CJ, Voors-Pette C, de Bruin TW (2004) Abdominal obesity and expression of familial combined hyperlipidemia. Obes Res 12: 2054-2061

[30] Shoulders CC, Jones EL, Naoumova RP (2004) Genetics of familial combined hyperlipidemia and risk of coronary heart disease. Hum Mol Genet 13 Spec No 1: R149-160

[31] Applebaum-Bowden D, McLean P, Steinmetz A, et al. (1989) Lipoprotein, apolipoprotein, and lipolytic enzyme changes following estrogen administration in postmenopausal women. J Lipid Res 30: 1895-1906

[32] Lawlor DA, Ebrahim S, Smith GD (2003) The association of socio-economic position across the life course and age at menopause: the British Women's Heart and Health Study. Bjog 110: 1078-1087

[33] Bos G, Snijder MB, Nijpels G, et al. (2005) Opposite contributions of trunk and leg fat mass with plasma lipase activities: the Hoorn study. Obes Res 13: 1817-1823 
3.

General discussion

Discussion of specific aims Methodological considerations Concluding remarks en future perspectives 


\subsection{Discussion of specific aims}

The results of this thesis will be discussed according to the specific aims that were posed in the introduction (paragraph 1.4)

\subsubsection{Genetic predisposition to develop fatty liver in FCHL: possible mechanisms}

Since FCHL patients are more prone to develop hyperlipidemia, even after correction or matching for the degree of obesity [1,2], we questioned whether the same is true for the development of fatty liver. In a cross-sectional study, we showed in paragraph 2.1.1 that the BMI-adjusted prevalence of fatty liver measured by ultrasound was increased in FCHL patients as well as in their normolipidemic relatives when compared to spouses. Paragraph 2.1.2 revealed that fatty liver, as estimated from plasma ALT levels used as a biomarker, was related to (abdominal) obesity over a time period of five years. Of interest, for every degree of (abdominal) obesity, both FCHL patients and their normolipidemic relatives displayed higher ALT levels in comparison with their spouses. Both paragraphs clearly show, by utilizing different measures of fatty liver (ultrasound and ALT) and with different study designs (cross-sectional and longitudinal), that FCHL patients are more prone to develop fatty liver, even after correction for obesity. These findings are very suggestive of an increased genetic susceptibility to develop fatty liver, since FCHL patients were compared with their spouses, who share the same environment. Furthermore, the increased prevalence of fatty liver in normolipidemic relatives suggests that fatty liver may precede the hyperlipidemia in FCHL.

A genetic component responsible for the increased prevalence of fatty liver was eventually demonstrated in paragraph 2.1.3: 20 to $36 \%$ of the variability in fatty liver in FCHL is accounted for by genetic factors.

An increased genetic susceptibility to develop fatty liver in FCHL could have its mechanistic origin in all those processes that result in an increased hepatic fat accumulation. These processes are summarized in Figure 1 and will be reviewed below. The chromosomal regions which harbor the genes for these enzymes and hormones that are likely to be involved in the development of fatty liver are exhibited in Appendix B with the purpose of a direct comparison with linkage results for fatty liver in FCHL (presented in paragraph 2.1.3).

\subsubsection{Afferent causes}

In patients with nonalcoholic fatty liver disease (NAFLD) it has been estimated that almost twothirds of the hepatic fat input is derived from free fatty acids (FFA) [3].

The contribution of dysfunctional adipose tissue to an increased hepatic flux in FCHL has already been discussed in detail in the Introduction section (paragraph 1.2.1.1). Of interest, our laboratory recently reported an up-regulation of the fatty acid transporter CD36 in cultured pre-adipocytes from FCHL patients [4]. This further underlines the presence of a primary defect in FCHL adipose tissue rather than an adaptive phenomenon, since these pre-adipocytes were taken from their original environment and were subsequently cultured under standardized conditions [4]. 


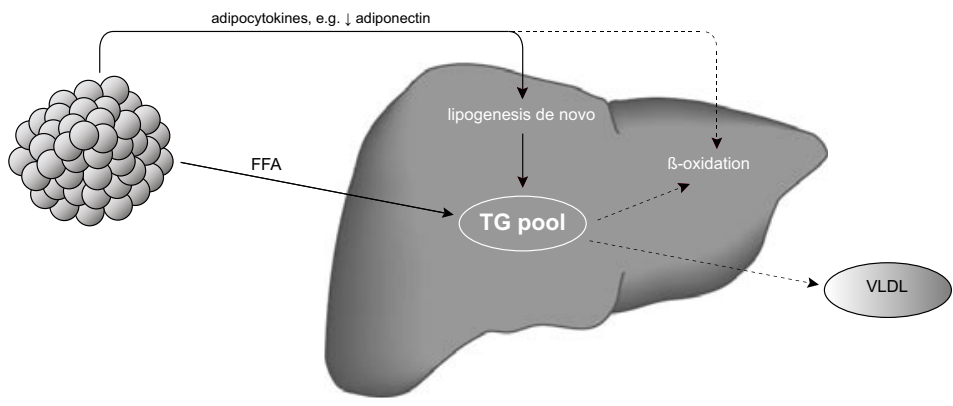

Figure 1.

Processes that could theoretically contribute to an increased susceptibility to develop fatty liver in FCHL. Dashed lines indicate suppression of a pathway, whereas straight lines represent stimulatory actions, all resulting in hepatic fat accumulation

The contribution of the different fat compartments to the hepatic flux of FFAs in humans has elegantly been addressed by Nielsen et al. [5]. By using stable isotope dilution and hepatic vein catherization techniques it was shown that the hepatic flux of FFAs was $20 \%$ greater in obese subjects [5]. Furthermore, the contribution of the visceral fat compartment to the flux ranged from $5 \%$ in lean subjects to $20 \%$ in obese subjects [5]. The remaining greater part was accounted for by the subcutaneous fat compartment [5]. These interesting results substantiate the crosssectional relationships of fatty liver with the degree of subcutaneous and visceral obesity in FCHL family members, as detailed in paragraph 2.1.1.

In addition to its energy storage function, adipose tissue has recently been recognized as an endocrine organ that is able to secrete many hormones, the so-called adipokines, such as leptin, adiponectin, resistin, tumor necrosis factor $\alpha(\mathrm{TNF} \alpha)$, interleukin 6 (Il- 6$)$, plasminogen activator inhibitor 1 (PAI-1), acylation stimulation protein, angiotensin and tissue factor [6]. The former two will be discussed in relation to the increased susceptibility to develop fatty liver in FCHL, since these two adipokines have been investigated in most detail in both FCHL and fatty liver:

- Leptin regulates energy homeostasis by reducing food intake and increasing energy expenditure [7]. Leptin deficient mice (ob/ob mice) are massively obese and display fatty liver and overproduction of VLDL-particles, which are, at least in part, due to an increased enzymatic activity of hepatic stearoyl-CoA desaturase-1 (SCD-1), a key enzyme in de novo lipogenesis (see below) [8]. Most of leptin's effects are centrally mediated by the leptin receptor, located in the hypothalamus, among others by inhibiting the release of neuropeptide Y (NPY) [9, 10]. Intra-cerebral administration of NPY in mice results in an increased hepatic glucose output and overproduction of VLDL-particles [11].

Paradoxically however, obese human subjects present with elevated rather than reduced plasma leptin levels, which is suggestive of a leptin-resistant state [12]. A very similar positive correlation between plasma leptin and the degree of obesity has been observed in FCHL [13]. In addition, there is some evidence that variants in the leptin receptor 
contribute the FCHL phenotype, further strengthening the evidence for involvement of this regulating pathway [14-16].

- Adiponectin is negatively associated with the degree of (abdominal) obesity, insulin levels and plasma triglycerides in both normal subjects and FCHL patients $[17,18]$, and with the amount of hepatic fat in patients with NAFLD [17].

Treatment with recombinant adiponectin alleviates both alcoholic and nonalcoholic fatty liver disease in mice by stimulating $\beta$-oxidation and suppressing de novo lipogenesis (via AMPK, see below) [19]. Decreased plasma levels of adiponectin may therefore also contribute to the increased susceptibility to develop fatty liver in FCHL. Of interest, FCHL patients exhibit decreased plasma levels of adiponectin, even after correction for (abdominal) obesity [18].

\subsubsection{Cellular imbalance}

Hepatic de novo lipogenesis and $\beta$-oxidation are tightly balanced, as shown in Figure 2. It has been estimated that almost one-third of the hepatic fat input arises from de novo lipogenesis [3]. A dysbalanced system in favor of an increased de novo lipogenesis could account for the increased genetic susceptibility to develop fatty liver in FCHL.

At present, data on the role of de novo lipogenesis and $\beta$-oxidation in the pathogenesis of FCHL are scarce:

- Hyperinsulinemia; insulin stimulates de novo lipogenesis and inhibits $\beta$-oxidation, thereby promoting the development of hepatic fat accumulation. Mice studies have shown that the presence of insulin resistance does not impair the action of insulin on these two processes [20]. A hyperinsulinemic state secondary to insulin resistance, as observed in FCHL [21$24]$, is therefore expected to result in an increased de novo lipogenesis and decreased $\beta$-oxidation.

- Variants in the upstream transcription factor 1 (USF1) gene; several genome scans have revealed significant linkage of the 1q21-23 region with FCHL-related traits in different FCHL populations [25-27]. Pajukanta et al. recently reported a significant association of variants in the USF1 gene, which resides in the 1q21-23 region, with plasma triglycerides in Finnish FCHL men [28]. This association was subsequently replicated in a Mexican FCHL population [29]. USF1 targets many genes with important roles in lipid and glucose metabolism [30], among others by stimulating fatty acid synthase (FAS), a key enzyme in lipogenesis [30]. However, our linkage analysis did not report a QTL for fatty liver in the 1q21-23 region (paragraph 2.1.3 and appendix A). Further study is required as this could be due to either a type II statistical error caused by the relatively small sample of sibling pairs that was used in our study (despite the use of a more refined phenotype). Alternatively, and perhaps more likely, the data can point to the fact that USF1 is implicated in the pathogenesis of FCHL through other pathways such as adipose tissue dysfunction than increased susceptibility to develop fatty liver [31].

- Variants in the peroxisome proliferator-activated receptor (PPAR $\alpha$ ) gene; although genetic variants in PPAR $\alpha$, an important activator of $\beta$-oxidation, have not been associated with FCHL $[32,33]$, it has been suggested that these variants could modify the FCHL phenotype, 


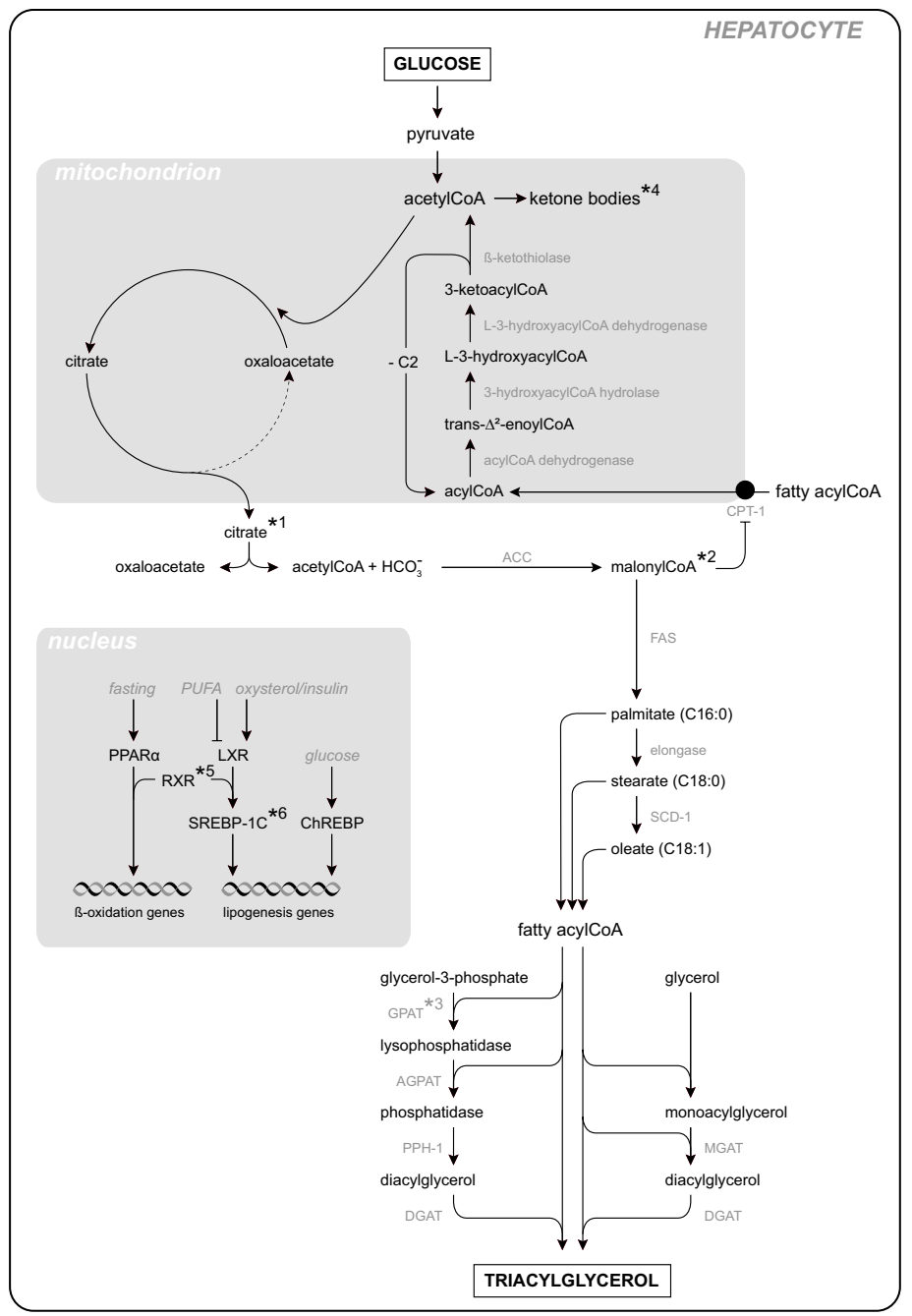

Figure 2.

Biochemical pathways of hepatic de novo lipogenesis and $\beta$-oxidation

*1 Citrate only leaves the mitochondrion when it is abundant, i.e. in the postprandial state

*2 MalonylCoA inhibits the fatty acid transporter CPT-1 and thereby $\beta$-oxidation

*3 GPAT is also present as a mitochondrial variant located in close vicinity of CPT-1 on the outer mitochondrial membrane. It scavenges fatty acids away from CPT- 1 and thereby inhibits $\beta$-oxidation

*4 Ketone bodies leave the hepatocyte and serve as a substrate in other organs, e.g. the brains

*5 RXR forms heterodimers with either PPAR $\alpha$ or LXR, thereby stimulating either $\beta$-oxidation or de novo lipogenesis, respectively

*6 SREBP-1C expression is suppressed by AMPK 
among others by influencing apolipoprotein CIII levels [32]. This modifying effect appears to be minor, at least for the increased susceptibility to develop fatty liver, since no QTL for fatty liver was observed in the 22q13.3 region (Appendix A).

- Variants in the retinoid X receptor gamma (RXR $\gamma)$ gene; variants in the $\mathrm{RXR} \gamma$ gene, located on chromosome 1q21-23, have been associated with FCHL and with hypertriglyceridemia, reduced HDL-cholesterol levels and decreased LPL activity/mass in patients with coronary artery disease [33]. However, it is unlikely that these variants also contribute to an increased susceptibility to develop fatty liver, since RXR $\gamma$ is particularly expressed in adipose tissue and muscle [33]. Of note, the same researchers demonstrated that variants in the liver X receptor alpha $(\mathrm{LXR} \alpha)$ gene, a gene that is expressed in hepatocytes, was not associated with FCHL [33].

- $\quad$ Stearoyl CoA desaturase (SCD1) activity; SCD1 catalyzes the introduction of the first double bond in the $\Delta 9$ position in several fatty acids, resulting in the formation of monounsaturated fatty acids. The preferred substrates are palmitate (16:0) and stearate (18:0), which are converted to palmitoleate (16:1) and oleate (18:1), respectively [34]. SCD1 appears to play an important role in the induction of fatty liver and obesity, as was shown in leptin deficient mice [8]. Of interest, Attie et al. found that the hepatic SCD1 activity in mice was reflected by the ratio of monounsaturated to saturated fatty acids in plasma ('the desaturation index') [35]. Of additional interest, this desaturation index, in particular the 18:1/18:0 ratio, was well correlated with plasma triglycerides in healthy human subjects $\left(\mathrm{r}^{2}=0.53, \mathrm{p}<0.001\right)$, but not in hypertriglyceridemic FCHL patients $\left(\mathrm{r}^{2}=0.02, \mathrm{p}=0.13\right)$ [35]. The heterogeneous nature of the hypertriglyceridemic FCHL phenotype, as outlined in paragraph 2.2.1, could account for this observation. In other words: it is expected that the desaturation index is perhaps more directly related to the amount of hepatic fat than to plasma triglycerides. Those patients who are hypertriglyceridemic due to an impaired clearance (and thus without fatty liver) are anticipated to have a low desaturation index, which explains the absent relation with plasma triglycerides.

- Increased postprandial ketone bodies; a decreased $\beta$-oxidation, which could theoretically account for the increased susceptibility to develop fatty liver in FCHL, is anticipated to result in decreased plasma ketone body levels (Figure 2). One study in the early nineties indeed showed that obese subjects with fatty liver presented with significantly lower plasma ketone bodies than obese subjects without fatty liver [36]. However, data on ketone bodies in FCHL are not conclusive: postprandial levels are elevated in FCHL [37, 38], consistent with increased $\beta$-oxidation possibly due to an increased supply of FFAs, whereas ketone bodies are reduced in the postabsorptive state, which could be the consequence of the hyperinsulinemic state [37].

\subsubsection{Efferent causes}

The production of VLDL-particles starts with the translation of the apolipoprotein B molecule. During translation, lipids are added to the nascent apolipoprotein B molecule by microsomal triglyceride transfer protein (MTP), which prevents the proteasomal degradation of apolipoprotein B that would otherwise occur. Apolipoprotein B can also be degraded by a 
non-proteasomal process, among others by insulin [39]. Further MTP-mediated lipid enrichment of apolipoprotein B takes place in the endoplasmatic reticulum. The production and secretion of buoyant apolipoprotein B lipoproteins, i.e. VLDL1-particles, is also inhibited by insulin [39]. Since FCHL patients have elevated insulin levels [21-24] and overproduce VLDL-particles [4044], it is expected that the process of VLDL-production is resistant to the action of insulin in these subjects. Mice studies have recently demonstrated that this hepatic insulin resistance is the consequence of an increased amount of hepatic fat [45].

Fatty liver has been associated with variants in genes that encode for proteins involved in the VLDLproduction, such as MTP [46, 47]. This is also illustrated by familial hypobetalipoproteinemia (FHBL), an autosomal dominant disease that is caused, among others, by mutations in the apolipoprotein B gene [48]. FHBL patients with this mutation have an impaired VLDLproduction [49] and therefore present with fatty liver AND bypolipidemia [50]. Since FCHL patients are bypenlipidemic, it will be clear that it is unlikely that these genes play a role in the increased susceptibility to develop fatty liver AND byperlipidemia in FCHL

However, there can be other factors that may interact with both the production and the catabolism of VLDL-particles, which could be relevant for the development of both fatty liver and hyperlipidemia in FCHL. For instance, apolipoprotein $\mathrm{E}$ is involved, not only in the catabolism and clearance of remnants particles, but also in the production of VLDLparticles [51]. Mice transgenic for a mutated apolipoprotein E (apo E3-Leiden) are therefore byperlipidemic (caused by an impaired catabolism) AND have fatty liver (caused by an impaired production) [52]. In addition, humans homozygous for the apolipoprotein E2 isoform are often byperlipidemic [53] AND appear to have more fatty liver than controls (paragraph 2.2.2). Of note, E2 homozygosity is an exclusion criterion for FCHL to avoid the diagnosis of type III hyperlipidemia (paragraph 1.1).

\subsubsection{Fatty liver based subgroups within FCHL: implications for diagnosis, current treatment and cardiovascular risk}

Previous studies in normal subjects and patients with type 2 diabetes mellitus have shown that VLDL-overproduction is associated with hepatic fat accumulation [54]. We asked the question whether fatty liver, as a qualitative ultrasound marker of hepatic fat accumulation, would discriminate between overproduction of VLDL-particles and impaired remnant clearance, the two processes that contribute to hypertriglyceridemia and exaggerated postprandial lipemia in FCHL subjects (paragraph 1.2). In the future, this hypothesis can be verified by use of stable isotopes, the only method that is able to discern overproduction form impaired clearance.

In paragraph 2.2.1, we demonstrated that within the hypertriglyceridemic FCHL phenotype, subgroups with different metabolic characteristics can be identified, based on presence or absence of fatty liver. The present data should be regarded as hypothesis generating, justifying further laborious and expensive stable isotope studies. Another fruitful avenue of research can be expected in the field of biomarkers of hepatic de novo lipogenesis or $\beta$-oxidation, for instance SCD-activity and ketone bodies, in both hypertriglyceridemic subgroups.

Scientists and physicians would benefit from further delineation of the two subgroups that 
have been identified in paragraph 2.2.1. The presence of relatively homogeneous subgroups within FCHL should facilitate the elucidation of the complex genetic background of FCHL and would enable a targeted treatment strategy to prevent cardiovascular complications. The clinical relevance of these subgroups was further underlined in paragraph 2.2.2 by demonstrating that the cardiovascular risk profile regarding inflammation (CRP), endothelial function (E-selectin) and fibrinolysis (PAI-1) was different between the two subgroups.

The treatment strategy to prevent cardiovascular complications could be different for the two newly identified hypertriglyceridemic subgroups, given the (relatively) new medications that have been shown to alleviate fatty liver and its related features. A large clinical trial very recently demonstrated that the administration of rimonabant, a selective cannabinoid-1 receptor blocker, caused a decrease in obesity and a signficant improvement of plasma insulin and triglycerides levels, due to a decreased appetite [55]. Furthermore, thiazolidinediones, a more established group of medication in the treatment of type 2 diabetes mellitus, activate the peroxisome proliferator activated receptor gamma (PPAR $\gamma$ ) resulting, among others, in an impressive decrease in the hepatic fat accumulation [56, 57]. However, plasma triglycerides levels remain unchanged during treatment of FCHL and diabetes patients $[58,59]$. This deserves further study before this group of medication can be routinely used in the treatment of FCHL (subgroups).

\subsubsection{Fatty liver in relation to multiple-type byperlipidemia}

The presence of different lipid phenotypes not only within one family, but also within one individual [60-62] has complicated the elucidation of FCHL and has therefore prompted researchers to redefine FCHL using traits that are more consistent over time, such as apolipoprotein B [61]. The discussion is also confounded by the issue of optimizing the prediction of cardiovascular events, using plasma lipid parameters, apolipoprotein B, or other markers [63]. The low temporal variability of apolipoprotein B levels probably also explains why the most consistent QTL over time was found for this trait, as was shown in paragraph 2.3.1.

A better understanding of the multiple-type hyperlipidemia has the potential benefit of providing more insight in the complex pathogenesis of FCHL. Previous studies have demonstrated that the switch in triglyceride phenotype, i.e. from normotriglyceridemia to hypertriglyceridemia or vice versa, is associated with changes in BMI and plasma insulin levels [61, 64]. In paragraph 2.1.2 we showed that changes in fatty liver, as estimated with ALT levels, were associated with the switch in triglyceride phenotype. As discussed in that same section, the absent relation of waist circumference and insulin levels with the switch in triglyceride phenotype is probably explained by a lack of statistical power. Of interest, Westerbacka et al. recently reported that a sudden increase or decrease in dietary fat intake can already influence the amount of hepatic fat without affecting the degree of obesity [65]. This indicates that fatty liver is a reversible phenomenon and that the switch in triglyceride phenotype can therefore easily occur.

The link between fatty liver and the switch in cholesterol phenotype is probably more distant and complex, as was shown in paragraph 2.3.2. Current knowledge on the production and catabolism of VLDL1- and VLDL2-particles led us to hypothesize that an increase in plasma triglycerides is accompanied by an increase in LDL-cholesterol, which attenuates and even inverts 
to a decrease, due to the formation of small-dense LDL particles, when plasma triglycerides (= VLDL1) rise further. Of interest, we have previously demonstrated that the combination of small-dense LDL and elevated plasma triglycerides, i.e. the atherogenic lipid phenotype, is indeed associated with an increased amount of VLDL1-particles in FCHL patients [66].

The parabolic relation between plasma triglycerides and LDL-cholesterol, which was observed in the male FCHL population, can explain, at least in part, the switch in cholesterol phenotype that has been observed in FCHL individuals [60-62]. This parabolic relation was not specific for the male FCHL population, since it was also found in the general population as well as in a diabetes population, which suggests that the 'multiple-type hyperlipidemia' within one individual is not a FCHL specific phenomenon. This is corroborated by the results presented in paragraph 2.1.2 where we demonstrated that the switch in triglyceride or cholesterol phenotype did not appear to occur more frequently in FCHL when compared to a (small) group of spouses. Our results therefore strongly suggest that the 'multiple-type hyperlipidemia' within one individual is not specific for FCHL. Previous reports on the switch in cholesterol and triglyceride phenotype in FCHL did not include a reference group and were therefore unable to address the FCHLspecificity of this phenomenon [61, 62, 64]. Delawi et al. did however use a reference group and were the first to show that the day-to-day variability in fasting lipids was not unique to FCHL patients but in fact occurred to the same extent in healthy controls [67].

\subsection{Methodological considerations}

There are several aspects of this thesis that deserve some critical notes:

- Genetic dependency and large pedigrees; our study population consisted of FCHL patients and their relatives, derived from approximately twenty well-defined FCHL families. Subjects were therefore not (genetically) independent, which could have interfered with the outcomes as presented in the current thesis. For example, a certain relationship that has been observed in our FCHL population could actually be explained by one large pedigree that is not necessarily a typical FCHL family. The problem of genetic dependency or family relatedness could theoretically be overcome by conducting these studies in genetically independent FCHL patients. However, this is virtually unfeasible in practice: 178 genetically independent FCHL patients (derived from 178 FCHL families) would be required to conduct a study as presented in paragraph 2.1.1.

Some research groups correct for genetic dependency in their statistical analyses by including family number as a random factor in the regression model $[18,61]$, which is basically the same as including each family as a dummy variable in the regression analysis, with the only exception that only one degree of freedom is required for the former method and $\mathrm{n}-1$ degrees of freedom are required for $\mathrm{n}$ families for the latter method. However, the FCHL population in the current thesis contained pedigrees of very diverse sizes, ranging from 2 to 32 relatives per family. This is not corrected for by these statistical methods, which do not take into account the weight of the individual pedigrees.

In paragraph 2.1.1 we ruled out familial bias as an explanation for the increased prevalence of fatty liver in FCHL by showing that a similar trend could be discerned when only one 
individual from each family was taken for analysis.

The subgroups that were identified based on the presence or absence of fatty liver in paragraph 2.2.1 and paragraph 2.2.2 are not explained by one or two large families, since the 13 hypertriglyceridemic subjects without fatty liver were derived from 10 different families and the 25 hypertriglyceridemic subjects with fatty liver from 17 different families.

The parabolic relation between plasma triglycerides and LDL-cholesterol in all FCHL family members, as presented in paragraph 2.3.2, was also present when the Nijmegen and Maastricht populations were analyzed separately.

Although genetic dependency is by definition required for heritability estimations and linkage analyses, one large family could still account for the greater part of the outcomes. One strategy to circumvent this potential hazard is to statistically correct for pedigree size, i.e. by weighed analysis. However, the amount of sib pairs and pedigrees in our study was relatively low to have enough statistical power. We therefore used an alternative strategy in paragraph 2.1.3, i.e. by re-analyzing our QTL without the potentially influential, large pedigree. Since very similar, somewhat less significant outcomes were obtained when this particular family was excluded from analyses, we are confident that the observed QTL can be applied to FCHL in general, and not to one certain FCHL pedigree.

- FCHL patients versus normolipidemic relatives; a recurring dilemma is how to deal with normolipidemic relatives in statistical analyses. Should they be analyzed together with FCHL patients, or alternatively: should they be regarded as a separate group?

Since the FCHL phenotype is the consequence of a complex interaction between genetic susceptibility and unfavorable lifestyle habits [2], it is expected that all patients are genetically predisposed. The normolipidemic relatives most likely comprise a heterogeneous group of individuals: some will be genetically susceptible, but not exposed to an unhealthy lifestyle to develop hyperlipidemia whereas others are not susceptible. Many research groups have conducted their analyses separately for FCHL patients and normolipidemic relatives $[18,68]$. In this thesis, we have often chosen to perform a combined analysis of FCHL patients and their normolipidemic relatives and included the affected state (patient versus normolipidemic relative) as a covariate.

The absent difference in the prevalence of fatty liver between FCHL patients and their normolipidemic relatives after correction for age, gender and BMI in paragraph 2.1.1 supports that strategy. These outcomes emphasize the necessity to monitor normolipidemic relatives in clinical practice, since they are at increased risk to develop fatty liver and therefore hyperlipidemia.

- $\quad$ Spouses as a reference group; in the current thesis, the spouses of the FCHL patients and relatives were included as an unselected and unrelated reference group. The advantage of such a reference group, besides the practical convenience of inviting FCHL patients together with their spouses for a visit to our research clinic, is that FCHL patients, relatives and spouses are exposed to a similar environment. In this way environmental factors play a minor role as a possible cause of an observed statistical difference between patients/ relatives and spouses. It should however be noted that spouses do not necessarily represent 
a random sample of the general population.

- Two weeks withdrawal from lipid lowering medication; FCHL patients were withdrawn from their lipid lowering medication two weeks prior to their visit to be able to study their untreated lipid levels. For ethical considerations we tried to keep this period as short as possible, but long enough for the medication to be fully eliminated from the body. A two-week period appeared to be the optimal time span: the pharmacological half time of statins varies from 2 hours (pravastatin) to 19 hours (rosuvastatin)[69]. The expected $100 \%$ elimination will thus be reached after 4 days at maximum ( $=5$ times 19 hours), leaving another 10 days for the LDL-receptor expression to be normalized. The half life of gemfibrozil, the most frequently prescribed fibrate, is 1.5 hour [69]. Complete washout will be reached within one day, remaining another 13.5 days for PPAR $\alpha$ receptors to function normally, which is probably long enough. In contrast, the half life of ciprofibrate is 90 hours [69], thus taking 19 days ( $=5$ times 90 hours) for this drug to be eliminated, which is longer than 14 days. However, it is not likely that this has markedly influenced our data, since only 3 subjects used ciprofibrate. Moreover, these subjects were not accidentally misclassified as normolipidemic, since their plasma triglycerides levels were elevated.

- Surrogates of fatty liver; in the current thesis we used surrogates markers for the presence or absence of fatty liver, such as liver ultrasound and ALT levels. Gold standard measures of hepatic fat accumulation such as liver biopsies were either ethically unacceptable or, in the case of MR spectroscopy of CT scanning, too expensive. We have obtained data that provide us with confidence that the obtained results are adequate and reliable for the intended purpose, since outcomes for both surrogates (ultrasound and ALT) were very comparable: both surrogates showed that the prevalence of fatty liver is increased in both FCHL patients and their normolipidemic relatives (paragraph 2.1.1 and 2.1.2). The relation of the different ultrasonographical stages of fatty liver with plasma levels of apolipoprotein B, total cholesterol and triglycerides was very similar when plasma ALT levels were used instead (paragraph 2.1.3). Finally, the linkage plots were generally in agreement for both surrogates (paragraph 2.1.3 and Appendix A).

- Sex difference; some of our results were only observed in the male population (paragraph 2.1.3), or were different between men and women (paragraph 2.3.2), which is not surprising given the numerous effects that estrogens exert on liver and lipid metabolism, as addressed in detail in those particular paragraphs. It would be preferable to conduct all analyses split by sex, since correcting for sex, by including sex as a variable in a regression model, does not correct for possible complex interactions between sex and a certain variable nor does it correct for the noise that is present within one sex group, which is probably the case in the female group, due to the presence of premenopausal women (with different menstrual stages) and postmenopausal women. However, due to a lack of power, we were sometimes obliged to conduct our analyses combined for men and women with correction for sex. 


\subsection{Concluding remarks and future perspectives}

The current thesis can be summarized in one figure, as shown in Figure 3. The figure shows the $95 \%$ confidence intervals of the longitudinal parabolic relation between plasma triglycerides and LDL-cholesterol in the FCHL men (paragraph 2.3.2). An increase in plasma triglycerides is related with (and thought to be the consequence of) an increased hepatic fat accumulation (paragraph 2.1.1 and 2.1.2), which is in part genetically determined (paragraph 2.1.3). In addition, other factors besides fatty liver are expected to play a role in the development of hypertriglyceridemia as well (paragraph 2.2.1). The parabolic relation of plasma triglycerides and LDL-cholesterol can explain, at least in part, the multiple-type hyperlipidemia in FCHL.

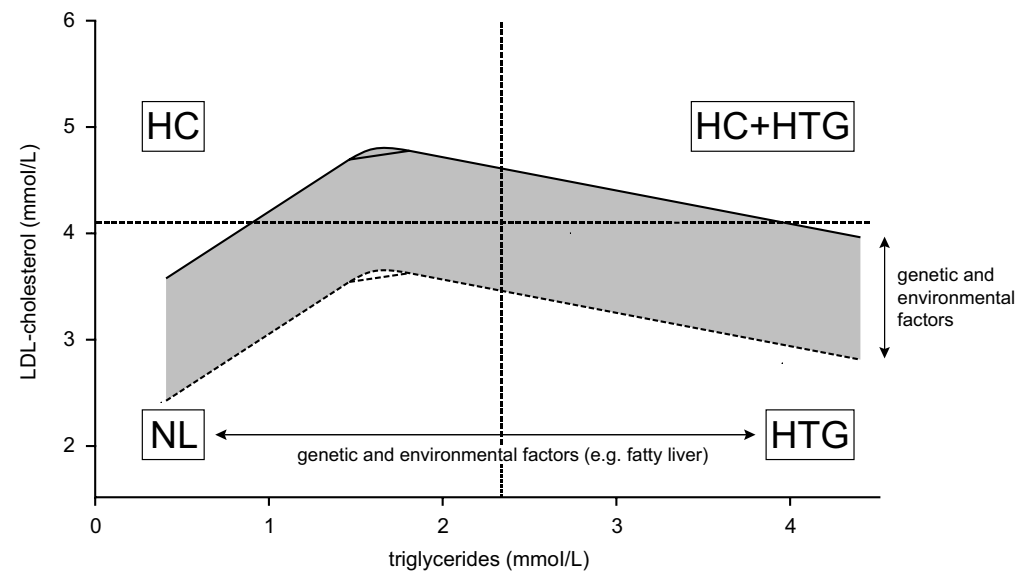

Figure 3.

Parabolic relation between plasma triglycerides and LDL-cholesterol in the male Maastricht/Nijmegen FCHL population as presented in paragraph 2.3.2. The upper straight and lower dashed line (covering the gray area) indicate the 95th percent confidence interval. The horizontal and vertical lines represent the cut-off value for high LDL-cholesterol and triglycerides levels, respectively, as defined by the ATPIII criteria. Abbreviations: NL: normolipidemia, HC: hypercholesterolemia, HTG: hypertriglyceridemia.

It would be of interest to investigate the relation between fatty liver, quantitatively measured with magnetic resonance spectroscopy, and the production of VLDL, as determined with stable isotopes, in particularly hypertriglyceridemic FCHL patients. Such a relation, which has already been demonstrated in normal subjects and patients with type 2 diabetes mellitus [54], would definitely provide evidence that one of the best documented features of FCHL, i.e. VLDLoverproduction [40-44], is related to the amount of hepatic fat. This definite proof, combined with the data presented in the current thesis, would justify a shift in research focus from causes of postprandial lipemia and hyperlipidemia to causes of hepatic fat accumulation and organ specific insulin resistance in FCHL. In addition, such a study would also give a more definite 
answer to whether the hypertriglyceridemia in the subgroups with and without fatty liver, as presented in paragraph 2.2.1, is really of different origin. Such a difference could have marked implications for the clinical approach of a FCHL patient with respect to diagnosis, cardiovascular risk estimation and treatment, as outlined in detail in paragraph 3.1.2.

Another aspect of this thesis that deserves further attention is our observation that the multipletype hyperlipidemia does not appear to be a FCHL-specific phenomenon.

In the early seventies, Goldstein, Nikkila and Rose independently reported the presence of a new, familial dyslipidemia that was supposed to be distinct from the already existing familial hypercholesterolemia and familial hypertriglyceridemia [70-72]. This new entity, familial combined hyperlipidemia, was characterized by the presence of different Fredrickson's types of hyperlipidemia within one family [70-72] and, as was demonstrated ten years later, within one individual [60-62]. One can already anticipate that, if patients from an established FCHL family switch lipid phenotype over time, they could coincentally be all hypercholesterolemic at one time point and hypertriglyceridemic at another measurement. Therefore, the diagnosis of FCHL very much depends on the moment a family is measured. Furthermore, this example already demonstrates that the different familial dyslipidemias, i.e. familial hypercholesterolemia (LDL-receptor and apolipoprotein B defects excluded), familial combined hyperlipidemia and familial hypertriglyceridemia, are potentially overlapping in their presenting phenotype in a single individual. Hence, it would be of particular interest to investigate whether a similar parabolic relation between plasma triglycerides and LDL-cholesterol, due to the development of the atherogenic lipid profile, would be observed in the other familial dyslipidemias. This would reveal, when affirmative, that the 'multiple-type hyperlipdemia' can be present not only in the general population and type 2 diabetes mellitus, but also in all (familial) forms of dyslipidemia, which can have serious implications for our approach towards the familial dyslipidemias in general.

'the fact is that FCHL was named when the modern era of the investigation of the plasma lipoproteins was just beginning’ [73] 


\subsection{References}

[1] Ascaso JF, Sales J, Merchante A, et al. (1997) Influence of obesity on plasma lipoproteins, glycaemia and insulinaemia in patients with familial combined hyperlipidaemia. Int J Obes Relat Metab Disord 21: 360-366

[2] van der Kallen CJ, Voors-Pette C, de Bruin TW (2004) Abdominal obesity and expression of familial combined hyperlipidemia. Obes Res 12: 2054-2061

[3] Donnelly KL, Smith CI, Schwarzenberg SJ, Jessurun J, Boldt MD, Parks EJ (2005) Sources of fatty acids stored in liver and secreted via lipoproteins in patients with nonalcoholic fatty liver disease. J Clin Invest 115: 1343-1351

[4] Meex SJ, van der Kallen CJ, van Greevenbroek MM, et al. (2005) Up-regulation of CD36/FAT in preadipocytes in familial combined hyperlipidemia. Faseb J 19: 2063-2065

[5] Nielsen S, Guo Z, Johnson CM, Hensrud DD, Jensen MD (2004) Splanchnic lipolysis in human obesity. J Clin Invest 113: 1582-1588

[6] Pittas AG, Joseph NA, Greenberg AS (2004) Adipocytokines and insulin resistance. J Clin Endocrinol Metab 89: 447-452

[7] Halaas JL, Gajiwala KS, Maffei M, et al. (1995) Weight-reducing effects of the plasma protein encoded by the obese gene. Science 269: 543-546

[8] Cohen P, Miyazaki M, Socci ND, et al. (2002) Role for stearoyl-CoA desaturase-1 in leptin-mediated weight loss. Science 297: 240-243

[9] Erickson JC, Hollopeter G, Palmiter RD (1996) Attenuation of the obesity syndrome of $\mathrm{ob} / \mathrm{ob}$ mice by the loss of neuropeptide Y. Science 274: 1704-1707

[10] Cohen P, Zhao C, Cai X, et al. (2001) Selective deletion of leptin receptor in neurons leads to obesity. J Clin Invest 108: 1113-1121

[11] van den Hoek AM, Voshol PJ, Karnekamp BN, et al. (2004) Intracerebroventricular neuropeptide $\mathrm{Y}$ infusion precludes inhibition of glucose and VLDL production by insulin. Diabetes 53: 2529-2534

[12] Considine RV, Sinha MK, Heiman ML, et al. (1996) Serum immunoreactive-leptin concentrations in normal-weight and obese humans. N Engl J Med 334: 292-295

[13] van der Vleuten GM, Veerkamp MJ, van Tits LJ, et al. (2005) Elevated leptin levels in subjects with familial combined hyperlipidemia are associated with the increased risk for CVD. Atherosclerosis 183: 355-360

[14] van der Kallen CJ, Cantor RM, van Greevenbroek MM, et al. (2000) Genome scan for adiposity in Dutch dyslipidemic families reveals novel quantitative trait loci for leptin, body mass index and soluble tumor necrosis factor receptor superfamily $1 \mathrm{~A}$. Int J Obes Relat Metab Disord 24: 1381-1391

[15] Allayee H, Krass KL, Pajukanta P, et al. (2002) Locus for elevated apolipoprotein B levels on chromosome 1p31 in families with familial combined hyperlipidemia. Circ Res 90:926931

[16] van der Vleuten GM, Kluijtmans LA, Hijmans A, Blom HJ, Stalenhoef AF, de Graaf J (2006) The Gln223Arg polymorphism in the leptin receptor is associated with familial combined hyperlipidemia. Int J Obes (Lond) 30: 892-898 
[17] Bugianesi E, Pagotto U, Manini R, et al. (2005) Plasma adiponectin in nonalcoholic fatty liver is related to hepatic insulin resistance and hepatic fat content, not to liver disease severity. J Clin Endocrinol Metab 90: 3498-3504

[18] van der Vleuten GM, van Tits LJ, den Heijer M, Lemmers H, Stalenhoef AF, de Graaf J (2005) Decreased adiponectin levels in familial combined hyperlipidemia patients contribute to the atherogenic lipid profile. J Lipid Res 46: 2398-2404

[19] Xu A, Wang Y, Keshaw H, Xu LY, Lam KS, Cooper GJ (2003) The fat-derived hormone adiponectin alleviates alcoholic and nonalcoholic fatty liver diseases in mice. J Clin Invest 112: $91-100$

[20] Horton JD, Goldstein JL, Brown MS (2002) SREBPs: activators of the complete program of cholesterol and fatty acid synthesis in the liver. J Clin Invest 109: 1125-1131

[21] Aitman TJ, Godsland IF, Farren B, Crook D, Wong HJ, Scott J (1997) Defects of insulin action on fatty acid and carbohydrate metabolism in familial combined hyperlipidemia. Arterioscler Thromb Vasc Biol 17: 748-754

[22] Pihlajamaki J, Karjalainen L, Karhapaa P, Vauhkonen I, Laakso M (2000) Impaired free fatty acid suppression during hyperinsulinemia is a characteristic finding in familial combined hyperlipidemia, but insulin resistance is observed only in hypertriglyceridemic patients. Arterioscler Thromb Vasc Biol 20: 164-170

[23] van der Kallen CJ, Voors-Pette C, Bouwman FG, et al. (2002) Evidence of insulin resistant lipid metabolism in adipose tissue in familial combined hyperlipidemia, but not type 2 diabetes mellitus. Atherosclerosis 164: 337-346

[24] Bredie SJ, Tack CJ, Smits P, Stalenhoef AF (1997) Nonobese patients with familial combined hyperlipidemia are insulin resistant compared with their nonaffected relatives. Arterioscler Thromb Vasc Biol 17: 1465-1471

[25] Pajukanta P, Nuotio I, Terwilliger JD, et al. (1998) Linkage of familial combined hyperlipidaemia to chromosome 1q21-q23. Nat Genet 18: 369-373

[26] Coon H, Myers RH, Borecki IB, et al. (2000) Replication of linkage of familial combined hyperlipidemia to chromosome 1q with additional heterogeneous effect of apolipoprotein A-I/C-III/A-IV locus. The NHLBI Family Heart Study. Arterioscler Thromb Vasc Biol 20: 2275-2280

[27] Pei W, Baron H, Muller-Myhsok B, et al. (2000) Support for linkage of familial combined hyperlipidemia to chromosome 1q21-q23 in Chinese and German families. Clin Genet 57: 29-34

[28] Pajukanta P, Lilja HE, Sinsheimer JS, et al. (2004) Familial combined hyperlipidemia is associated with upstream transcription factor 1 (USF1). Nat Genet 36: 371-376

[29] Huertas-Vazquez A, Aguilar-Salinas C, Lusis AJ, et al. (2005) Familial combined hyperlipidemia in Mexicans: association with upstream transcription factor 1 and linkage on chromosome 16q24.1. Arterioscler Thromb Vasc Biol 25: 1985-1991

[30] Lee JC, Lusis AJ, Pajukanta P (2006) Familial combined hyperlipidemia: upstream transcription factor 1 and beyond. Curr Opin Lipidol 17: 101-109

[31] Naukkarinen J, Gentile M, Soro-Paavonen A, et al. (2005) USF1 and dyslipidemias: converging evidence for a functional intronic variant. Hum Mol Genet 14: 2595-2605 
[32] Eurlings PM, van der Kallen CJ, Geurts JM, Flavell DM, de Bruin TW (2002) Identification of the PPARA locus on chromosome $22 \mathrm{q} 13.3$ as a modifier gene in familial combined hyperlipidemia. Mol Genet Metab 77: 274-281

[33] Nohara A, Kawashiri MA, Claudel T, et al. (2007) High frequency of a retinoid X receptor gamma gene variant in familial combined hyperlipidemia that associates with atherogenic dyslipidemia. Arterioscler Thromb Vasc Biol 27: 923-928

[34] Ntambi JM (1999) Regulation of stearoyl-CoA desaturase by polyunsaturated fatty acids and cholesterol. J Lipid Res 40: 1549-1558

[35] Attie AD, Krauss RM, Gray-Keller MP, et al. (2002) Relationship between stearoyl-CoA desaturase activity and plasma triglycerides in human and mouse hypertriglyceridemia. J Lipid Res 43: 1899-1907

[36] Inokuchi T, Orita M, Imamura K, Takao T, Isogai S (1992) Resistance to ketosis in moderately obese patients: influence of fatty liver. Intern Med 31: 978-983

[37] Meijssen S, Cabezas MC, Twickler TB, Jansen H, Erkelens DW (2000) In vivo evidence of defective postprandial and postabsorptive free fatty acid metabolism in familial combined hyperlipidemia. J Lipid Res 41: 1096-1102

[38] Halkes CJ, Van Dijk H, Verseyden C, et al. (2003) Gender Differences in Postprandial Ketone Bodies in Normolipidemic Subjects and in Untreated Patients With Familial Combined Hyperlipidemia. Arterioscler Thromb Vasc Biol

[39] Fisher EA, Ginsberg HN (2002) Complexity in the secretory pathway: the assembly and secretion of apolipoprotein B-containing lipoproteins. J Biol Chem 277: 17377-17380

[40] Venkatesan S, Cullen P, Pacy P, Halliday D, Scott J (1993) Stable isotopes show a direct relation between VLDL apoB overproduction and serum triglyceride levels and indicate a metabolically and biochemically coherent basis for familial combined hyperlipidemia. Arterioscler Thromb 13: 1110-1118

[41] Chait A, Albers JJ, Brunzell JD (1980) Very low density lipoprotein overproduction in genetic forms of hypertriglyceridaemia. Eur J Clin Invest 10: 17-22

[42] Janus ED, Nicoll AM, Turner PR, Magill P, Lewis B (1980) Kinetic bases of the primary hyperlipidaemias: studies of apolipoprotein B turnover in genetically defined subjects. Eur J Clin Invest 10: 161-172

[43] Kissebah AH, Alfarsi S, Adams PW (1981) Integrated regulation of very low density lipoprotein triglyceride and apolipoprotein-B kinetics in man: normolipemic subjects, familial hypertriglyceridemia and familial combined hyperlipidemia. Metabolism 30: 856868

[44] Cortner JA, Coates PM, Bennett MJ, Cryer DR, Le NA (1991) Familial combined hyperlipidaemia: use of stable isotopes to demonstrate overproduction of very lowdensity lipoprotein apolipoprotein B by the liver. J Inherit Metab Dis 14: 915-922

[45] Samuel VT, Liu ZX, Qu X, et al. (2004) Mechanism of hepatic insulin resistance in nonalcoholic fatty liver disease. J Biol Chem 279: 32345-32353

[46] Namikawa C, Shu-Ping Z, Vyselaar JR, et al. (2004) Polymorphisms of microsomal triglyceride transfer protein gene and manganese superoxide dismutase gene in nonalcoholic steatohepatitis. J Hepatol 40: 781-786 
[47] Bernard S, Touzet S, Personne I, et al. (2000) Association between microsomal triglyceride transfer protein gene polymorphism and the biological features of liver steatosis in patients with type II diabetes. Diabetologia 43: 995-999

[48] Schonfeld G (2003) Familial hypobetalipoproteinemia: a review. J Lipid Res 44: 878-883

[49] Aguilar-Salinas CA, Barrett PH, Parhofer KG, et al. (1995) Apoprotein B-100 production is decreased in subjects heterozygous for truncations of apoprotein B. Arterioscler Thromb Vasc Biol 15: 71-80

[50] Tanoli T, Yue P, Yablonskiy D, Schonfeld G (2004) Fatty liver in familial hypobetalipoproteinemia: roles of the APOB defects, intra-abdominal adipose tissue, and insulin sensitivity. J Lipid Res 45: 941-947

[51] Mensenkamp AR, Jong MC, van Goor H, et al. (1999) Apolipoprotein E participates in the regulation of very low density lipoprotein-triglyceride secretion by the liver. J Biol Chem 274: 35711-35718

[52] Mensenkamp AR, van Luyn MJ, van Goor H, et al. (2000) Hepatic lipid accumulation, altered very low density lipoprotein formation and apolipoprotein E deposition in apolipoprotein E3-Leiden transgenic mice. J Hepatol 33: 189-198

[53] Mahley RW, Huang Y, Rall SC, Jr. (1999) Pathogenesis of type III hyperlipoproteinemia (dysbetalipoproteinemia). Questions, quandaries, and paradoxes. J Lipid Res 40: 19331949

[54] Adiels M, Taskinen MR, Packard C, et al. (2006) Overproduction of large VLDL particles is driven by increased liver fat content in man. Diabetologia: 1-11

[55] Despres JP, Golay A, Sjostrom L (2005) Effects of rimonabant on metabolic risk factors in overweight patients with dyslipidemia. N Engl J Med 353: 2121-2134

[56] Neuschwander-Tetri BA, Brunt EM, Wehmeier KR, Oliver D, Bacon BR (2003) Improved nonalcoholic steatohepatitis after 48 weeks of treatment with the PPARgamma ligand rosiglitazone. Hepatology 38: 1008-1017

[57] Yki-Jarvinen H, Sutinen J, Silveira A, et al. (2003) Regulation of plasma PAI-1 concentrations in HAART-associated lipodystrophy during rosiglitazone therapy. Arterioscler Thromb Vasc Biol 23: 688-694

[58] Abbink EJ, De Graaf J, De Haan JH, Heerschap A, Stalenhoef AF, Tack CJ (2006) Effects of pioglitazone in familial combined hyperlipidaemia. J Intern Med 259: 107-116

[59] van Wijk JP, de Koning EJ, Martens EP, Rabelink TJ (2003) Thiazolidinediones and blood lipids in type 2 diabetes. Arterioscler Thromb Vasc Biol 23: 1744-1749

[60] Brunzell JD, Albers JJ, Chait A, Grundy SM, Groszek E, McDonald GB (1983) Plasma lipoproteins in familial combined hyperlipidemia and monogenic familial hypertriglyceridemia. J Lipid Res 24: 147-155

[61] Veerkamp MJ, de Graaf J, Bredie SJ, Hendriks JC, Demacker PN, Stalenhoef AF (2002) Diagnosis of familial combined hyperlipidemia based on lipid phenotype expression in 32 families: results of a 5-year follow-up study. Arterioscler Thromb Vasc Biol 22: 274-282

[62] McNeely MJ, Edwards KL, Marcovina SM, Brunzell JD, Motulsky AG, Austin MA (2001) Lipoprotein and apolipoprotein abnormalities in familial combined hyperlipidemia: a 20 year prospective study. Atherosclerosis 159: 471-481 
[63] Barter PJ, Ballantyne CM, Carmena R, et al. (2006) Apo B versus cholesterol in estimating cardiovascular risk and in guiding therapy: report of the thirty-person/ten-country panel. J Intern Med 259: 247-258

[64] Veerkamp MJ, de Graaf J, Stalenhoef AF (2005) Role of insulin resistance in familial combined hyperlipidemia. Arterioscler Thromb Vasc Biol 25: 1026-1031

[65] Westerbacka J, Lammi K, Hakkinen AM, et al. (2005) Dietary fat content modifies liver fat in overweight nondiabetic subjects. J Clin Endocrinol Metab 90: 2804-2809

[66] Georgieva AM, van Greevenbroek MM, Krauss RM, et al. (2004) Subclasses of lowdensity lipoprotein and very low-density lipoprotein in familial combined hyperlipidemia: relationship to multiple lipoprotein phenotype. Arterioscler Thromb Vasc Biol 24: 744749

[67] Delawi D, Meijssen S, Castro Cabezas M (2003) Intra-individual variations of fasting plasma lipids, apolipoproteins and postprandial lipemia in familial combined hyperlipidemia compared to controls. Clin Chim Acta 328: 139-145

[68] Karjalainen L, Pihlajamaki J, Karhapaa P, Laakso M (1998) Impaired insulin-stimulated glucose oxidation and free fatty acid suppression in patients with familial combined hyperlipidemia: a precursor defect for dyslipidemia? Arterioscler Thromb Vasc Biol 18: $1548-1553$

[69] (2006) Farmacotherapeutisch kompas. Roto Smeets Utrecht, Utrecht

[70] Goldstein JL, Schrott HG, Hazzard WR, Bierman EL, Motulsky AG (1973) Hyperlipidemia in coronary heart disease. II. Genetic analysis of lipid levels in 176 families and delineation of a new inherited disorder, combined hyperlipidemia. J Clin Invest 52: 1544-1568

[71] Rose HG, Kranz P, Weinstock M, Juliano J, Haft JI (1973) Inheritance of combined hyperlipoproteinemia: evidence for a new lipoprotein phenotype. Am J Med 54: 148-160

[72] Nikkila EA, Aro A (1973) Family study of serum lipids and lipoproteins in coronary heart-disease. Lancet 1: 954-959

[73] Sniderman AD, Ribalta J, Castro Cabezas M (2001) How should FCHL be defined and how should we think about its metabolic bases? Nutr Metab Cardiovasc Dis 11: 259-273 
4.

Summary Samenvatting

Dankwoord Curriculum vitae Scientific output 


\section{Summary}

Familial combined hyperlipidemia (FCHL) is a highly prevalent genetic dyslipidemia that is characterized by the presence of different lipid phenotypes, i.e. hypercholesterolemia, hypertriglyceridemia or the combination of both, in one family and one individual over time. FCHL family members have an increased risk to develop premature myocardial infarction, i.e. before the age of 60 years. This increased risk combined with the high prevalence of FCHL in the general population underlines the clinical relevance of this disease.

Nowadays it is well accepted that FCHL is a complex disease, which implicates that the FCHL phenotype is caused by the interaction of susceptibility genes in combination with environmental factors. After years of extensive research, FCHL has been recognized as a variant of the metabolic syndrome: FCHL patients are in general obese, particularly in the abdominal region, are resistant to the actions of insulin and have high blood pressure. These factors have been associated with an additional cardiovascular risk, independent of the elevated plasma lipid levels.

It is anticipated that the hyperlipidemia in FCHL is the consequence of both an increased production of triglyceride rich (VLDL-) particles by the liver and an impaired particle clearance by peripheral tissues. To date, two factors have been related to hepatic VLDL-overproduction, i.e. an increased flux of free fatty acids to the liver and hepatic insulin resistance. There is growing evidence that these two factors are causally linked to each other by an increased amount of hepatic fat (hepatic steatosis or fatty liver). It is thought that an increased flux of free fatty acids from adipose tissue towards to the liver results in hepatic fat accumulation, which in turn leads to hepatic insulin resistance and subsequently to VLDL-overproduction and hyperlipidemia.

In the present thesis, we investigated the role of fatty liver in the pathogenesis of FCHL. This theme was dissected in three lines of research:

\section{Genetic susceptibility to develop fatty liver in FCHL}

Since FCHL patients are more prone to develop hyperlipidemia, even after correcting or matching for the degree of obesity, we questioned whether the same is true for the development of fatty liver. In a cross-sectional study, we showed in paragraph 2.1.1 that the BMI-adjusted prevalence of fatty liver measured by ultrasound was increased in FCHL patients as well as in their normolipidemic relatives when compared to spouses. Paragraph 2.1.2 revealed that fatty liver, as estimated from plasma ALT levels used as a biomarker, was related to (abdominal) obesity over a time period of five years. Of interest, for every degree of (abdominal) obesity, both FCHL patients and their normolipidemic relatives displayed higher ALT levels in comparison with their spouses. Both paragraphs clearly show, by utilizing different measures of fatty liver (ultrasound and ALT) and with different study designs (cross-sectional and longitudinal), that FCHL patients are more prone to develop fatty liver, even after correction for obesity. These findings are very suggestive of an increased genetic susceptibility to develop fatty liver, since FCHL patients were compared with their spouses, who share the same environment. A genetic component responsible for the increased prevalence of fatty liver was eventually demonstrated in paragraph 2.1.3: 20 to $36 \%$ of the variability in fatty liver in FCHL is accounted for by genetic factors. 


\section{Fatty liver based subgroups within familial combined byperlipidemia}

In this section, we asked the question whether fatty liver, as a qualitative ultrasound marker of hepatic fat accumulation, would discriminate between overproduction of VLDL-particles and impaired remnant clearance, the two processes that contribute to hypertriglyceridemia in FCHL subjects. In paragraph 2.2.1, we demonstrated that within the hypertriglyceridemic FCHL phenotype, subgroups with different metabolic characteristics can be identified, based on presence or absence of fatty liver. Whether these subgroups really represent overproduction and impaired clearance pathways requires further research. Of interest, in paragraph 2.2.2 we demonstrated that the cardiovascular risk profile regarding inflammation (CRP), endothelial function (E-selectin) and fibrinolysis (PAI-1) was different between the two subgroups.

\section{Fatty liver in relation to the multiple-type hyperlipidemia}

The presence of different lipid phenotypes not only within one family, but also within one individual has complicated the elucidation of FCHL and has therefore prompted researchers to redefine FCHL using traits that are more consistent over time, such as apolipoprotein B. In paragraph 2.3.1 we indeed demonstrated that the quantitative trait locus obtained for apoliprotein B levels was the most stable over time when compared to loci for plasma triglycerides and cholesterol levels.

A better understanding of the so-called multiple-type hyperlipidemia has the potential benefit of providing more insight in the complex pathogenesis of FCHL. In paragraph 2.1.2 we showed that changes in fatty liver, as estimated with ALT levels, were associated with the switch in triglyceride phenotype, i.e. the switch form normotriglyceridemia to hypertriglyceridemia or vice versa. The link between fatty liver and the switch in cholesterol phenotype is probably more distant and complex, as was shown in paragraph 2.3.2. In this paragraph we showed that changes in plasma triglycerides concentrations are accompanied by changes in LDL-cholesterol levels. This relation appeared to be parabolic rather than linear: at low plasma triglycerides, LDLcholesterol levels increased parallel to increasing plasma triglycerides, whereas a further increase in triglycerides was accompanied by a decrease in LDL-cholesterol concentrations. This relation appeared to be an universally occurring phenomenon, since it was also observed in the general population and patients with type 2 diabetes mellitus. These results clearly show that the liver is the driving force behind the 'multiple-type hyperlipidemia' in FCHL. They also suggest that the 'multiple-type hyperlipidemia' within one individual is not specific for FCHL.

It can be concluded that the fatty liver trait is an integral feature of FCHL, given the increased genetic susceptibility, the ability to refine the hypertriglyceridemic FCHL phenotype and the relation with the multiple-type hyperlipidemia within one individual. This thesis therefore justifies a shift in research focus from causes of hyperlipidemia to causes of hepatic fat accumulation in FCHL. In addition, the availability of new therapeutic agents that target fatty liver and associated metabolic factors places this thesis in a clinical perspective. 


\section{Samenvatting}

Familiaire gecombineerde hyperlipidemie (FGH) is een veel voorkomende erfelijke aandoening die gekenmerkt wordt door verschillende lipide fenotypes binnen een familie en een individu, namelijk: hypercholesterolemie, hypertriglyceridemie of de combinatie van beiden. FGH familieleden hebben een verhoogd risico een hartinfarct te ontwikkelen voor het zestigste levensjaar. Dit verhoogde risico in combinatie met de hoge prevalentie van FGH in de algemene bevolking benadrukt de klinische relevantie van deze aandoening.

FGH is een complexe aandoening, wat betekent dat het FGH fenotype veroorzaakt wordt door de interactie tussen een genetische gevoeligheid en bepaalde omgevingsfactoren, zoals een ongezonde levensstijl. $\mathrm{Na}$ jaren van uitgebreid onderzoek is het duidelijk dat FGH onderdeel uitmaakt van het metabool syndroom: FGH patiënten hebben in het algemeen overgewicht, met name in de buikregio, zijn ongevoelig voor de werking van het hormoon insuline en hebben een hoge bloeddruk. Deze factoren zijn geassocieerd met een verhoogd cardiovasculair risico, onafhankelijk van de verhoogde plasma lipide waarden.

De hyperlipidemie in FGH is waarschijnlijk het gevolg van een toegenomen produktie van vetrijke deeltjes (VLDL) door de lever en een verminderde klaring van deze deeltjes door perifere weefsels. Op dit moment zijn er twee factoren waarvan we weten dat ze samenhangen met een toegenomen VLDL-produktie: een toegenomen flux van vrije vetzuren van vetweefsel naar de lever en insuline resistentie van de lever. Er is steeds meer bewijs dat deze twee factoren met elkaar verbonden zijn door een toegenomen stapeling van vet in de lever. Een toegenomen flux van vrije vetzuren naar de lever resulteert in leververvetting dat leidt tot insuline-resisentie van de lever en vervolgens tot VLDL-overproduktie en hyperlipidemie.

In dit proefschrift is de rol van leververvetting in de pathogenese van FGH onderzocht. Dit onderwerp is onderverdeeld in drie onderzoekslijnen:

\section{Genetische gevoeligheid voor het ontwikkelen van leververvetting in FGH}

Aangezien FGH patiënten gevoeliger zijn om hyperlipidemie te ontwikkelen, zelfs na correctie of matchen voor de mate van obesitas, vroegen we ons af hetzelfde geldt voor het ontstaan van leververvetting. In een cross-sectionele studie lieten we in paragraaf 2.1.1 zien dat de BMIgecorrigeerde prevalentie van leververvetting (gemeten met echo) hoger was in zowel FGH patiënten als hun normolipidemische familieleden wanneer zij vergeleken werden met hun (niet verwante) partners. Paragraaf 2.1.2 toonde aan dat plasma ALAT waarden, gebruikt als een surrogaat voor leververvetting, geassocieerd waren met de buikomvang en de mate van overgewicht over een periode van vijf jaar. Het was fascinerend te zien dat plasma ALAT hoger waren in FGH patiënten en hun normolipidemische familieleden in vergelijking met hun partners, ongeacht de mate van overgewicht. Beide paragrafen tonen duidelijk aan, met verschillende maten voor leververvetting (echo en ALAT) en met verschillende studie-opzetten (cross-sectioneel en longitudinaal), dat FGH patiënten gevoeliger zijn voor het ontwikkelen van leververvetting. Deze bevindingen zijn zeer suggestief voor een genetische component, aangezien FGH patiënten vergeleken werden met hun partners, die min of meer dezelfde levensstijl hebben. Een genetische component werd definitief aangetoond in paragraaf 2.1.3: 20 
tot $36 \%$ van de variatie in leververvetting in FGH wordt verklaard door erfelijkheidsfactoren.

\section{Subgroepen binnen FGH gebaseerd op leververvetting}

In deze sectie vroegen we ons af of het mogelijk is om met behulp van leververvetting, gemeten als een kwalitatieve maat met echo, een onderscheid te maken tussen overproduktie van VLDL-deeltjes en een verminderde klaring, de twee processen die bijdragen aan de hypertriglyceridemie in FGH patiënten. In paragraaf 2.2.1 toonden we aan dat het mogelijk is om binnen het hypertriglyceridemische fenotype subgroepen te herkennen met verschillende metabole eigenschappen gebaseerd op de aan- of afwezigheid van leververvetting. Er is meer onderzoek nodig om zeker te weten of deze subgroepen nu overproduktie en verminderde klaring vertegenwoordigen. In paragraaf 2.2.2. lieten we zien dat het cardiovasculaire risico profiel verschillend is tussen deze twee groepen met betrekking tot ontsteking (CRP), endotheelfunctie (E-selektine) en fibrinolyse (PAI-1).

\section{Leververvetting in relatie tot het wisselende fenotype in FGH}

Het voorkomen van verschillende lipide fenotypes binnen een familie, maar nog belangrijker binnen een individuele FGH patiënt, is een factor die de opheldering van FGH sterk compliceert. Het heeft ertoe geleid dat onderzoekers geprobeerd hebben FGH opnieuw te definiëren met parameters die meer stabiel over de tijd zijn, zoals apolipoproteine B. In paragraaf 2.3.1 lieten we zien dat een quantitative trait locus voor apolipoproteine B inderdaad het meest stabiel over de tijd is in vergelijking met loci voor plasma triglyceriden en cholesterol.

Een beter begrip van het zogenoemde wisselende fenotype kan ertoe leiden dat de complexe pathogenese van FGH inzichtelijker gemaakt wordt. In paragraaf 2.3.2 werd aangetoond dat veranderingen in leververvetting (benaderd met plasma ALAT waarden) geassocieerd zijn met het wisselende triglyceride fenotype (van normotriglyceridemie naar hypertriglyceridemie of andersom). De relatie van leververvetting met het wisselende cholesterol fenotype is waarschijnlijk complexer en minder direct zoals aangetoond in paragraaf 2.3.2. In deze paragraaf lieten we zien dat veranderingen in plasma triglyceriden samengaan met veranderingen in LDL-cholesterol. Deze relatie bleek niet lineair maar parabolisch te zijn: bij lage plasma triglyceriden concentraties ging een stijging in triglyceriden samen met een stijging in LDL-cholesterol waarden, terwijl een verdere stijging geassocieerd werd met een daling in LDL-cholesterol. Deze relatie bleek niet specifiek te zijn voor FGH, het werd namelijk ook geoberveerd in de algmene bevolking en patiënten met ouderdomssuikerziekte. Deze resultaten tonen duidelijk aan dat de lever de drijvende kracht is achter het wisselende fenotype en suggereren dat dit fenomeen niet specifiek is voor FGH.

Er kan geconcludeerd worden dat leververvetting een centraal aspect is van FGH, gezien de genetische gevoeligheid, de mogelijkheid om subgroepen te herkennen en de relatie met het wisselende fenotype in een individu. Derhalve rechtvaardigt dit proefschrift een verschuiving van onze aandacht van oorzaken van hyperlipidemie naar oorzaken van leververvetting in FGH. De beschikbaarheid van nieuwe therapeutische middelen die leververvetting en geassocieerde metabole factoren bestrijden plaatsen dit proefschrift in een klinisch perspectief. 


\section{Dankwoord}

$\mathrm{Na}$ vier jaar full-time onderzoek en één jaar in de avonduren, weekenden en nachtdiensten de puntjes op de i gezet te hebben, is het dan toch zover gekomen: het proefschrift is af en een periode is ten einde gekomen. Het is zoals gewoonlijk met horten en stoten gegaan, mijn jeugdelijk enthousiasme heeft wat deukjes opgelopen, maar uiteindelijk ben ik er (wetenschappelijk) rijper uitgekomen.

Mijn dankwoord begint bij alle FGH familieleden en partners die hebben deelgenomen aan dit onderzoek. Jullie zijn letterlijk uit alle windstreken gekomen, soms met ingewikkelde constructies, zodat moeder of vader ook mee kon komen. Hoewel het langzaam gaat, komen we stapje voor stapje dichter bij de ontrafeling van deze ingewikkelde aandoening. Heel hartelijk bedankt, ik hoop dat jullie in de toekomst met dezelfde bereidwilligheid zullen deelnemen aan het onderzoek.

Beste Tjerk, het is jouw toewijding die ervoor gezorgd heeft dat ik vanaf jaar 3 van mijn geneeskunde opleiding met tussenpozen betrokken ben geweest bij het FGH-onderzoek en het vakgebied endocrinologie. Jouw enthousiasme en inventiviteit hebben aanstekelijk gewerkt. Ik hoop dat we in de toekomst contact blijven houden.

$\mathrm{Na}$ het vertrek van Tjerk heb jij, Coen, de taak als promotor in Maastricht probleemloos overgenomen. Hoewel het een hele andere manier van werken was, heb ik veel van je geleerd op het gebied van structuur en prioriteiten. Als assistent in opleiding tot specialist hoop ik in de toekomst nog veel van je te kunnen leren.

Marleen en Carla, mijn co-promotores, ik begrijp maar niet hoe jullie het geduld hebben kunnen opbrengen wanneer ik weer eens jullie kamer kwam binnenstormen met een nieuwe hypothese of onderzoeksplan. Jullie zijn de continue, stabiele factor geweest tijdens mijn project. Heel hartelijk bedankt voor de dagelijkse begeleiding.

Dan mijn paranimfen, Joséphine en Dennis. Beste Joséphine, het is ondenkbaar jou niet als paranimf te hebben. Als team keken we wat glazig om ons heen waneer er tijdens de werkbesprekingen weer eens werd gesproken over 'PBS', 'bandjes' of de 'imager'. Als team hebben we het FGH project afgerond. In het lab ben jij de spil van het humane onderzoek en de inzet waarmee jij dit doet wordt maar al te vaak onterecht als een vanzelfsprekendheid gezien. Dennis, we zijn als AIO-collega's begonnen en ik ben blij dat we nu met elkaar kunnen werken als AIOS-collega's. Je laat je niet laat meeslepen door de dagelijkse beslommeringen en daar bewonder ik je om. Ik ben vereerd met jou als paranimf.

Dear Anna, regard this thesis as your second thesis. The outcomes of your thesis and our endless discussions in the evenings, on our way home and along the beautiful streets of Prague have resulted in this thesis. Although I initially thought otherwise, I think you have made the right 
decision after your promotion. I sincerely hope to keep in touch in the future.

Beste Steven, vanaf dag één hebben we samen het gehele AIO-traject doorlopen en dat heeft geresulteerd in een waardevolle vriendschap. Gaandeweg bemerkten we dat we in veel eigenschappen overeenkomen wat betreft enthousiasme, discipline, koppigheid en een stukje hoogheidswaan. Elk discipline kent zijn talenten en wat mij betreft bij jij het talent van de wetenschap. Jij weet precies wat er nodig is om een project tot een goed einde te leiden, en daar benijd ik je om. Ik hoop dat de wetenschap zich hiervan bewust is en je niet zal opgeven.

Hoeveelheden van één mililiter pipetteren was geen probleem, maar het echte werk is gedaan door Vicky en Margee. Heel hartelijk dank voor al jullie ondersteuning in het lab!

De metingen zouden niet mogelijk zijn geweest zonder de studenten Janine, Jaap, Jacco, Simon, Pauline en Caroline.

Dr. C.G. Schalkwijk, beste Casper, je bent als hoofd van het laboratorium aangetreden op het moment dat mijn project als ver gevorderd was. Hoewel we inhoudelijk niet veel met elkaar te maken hebben gehad, ben ik je dankbaar voor je werk op de achtergrond.

Prof. Dr. N.C. Schaper, beste Nicolaas, ik ben blij dat je deel uitmaakt van mijn leescommissie en hoop in de toekomst nog veel van je te kunnen leren.

Samenwerking is essentieel in de wetenschap. Het is naïef te denken dat je het allemaal in je eentje kunt oplossen. Mijn dank gaat daarom uit naar de volgende mensen:

Monique Bilderbeek-Beckers, jouw bijdrage als onafhankelijke observer van alle leverecho's is essentieel geweest voor dit proefschrift!

Prof. Dr. Ir. A.P. Hoeks, Dr J.M. Meinders en Jeroen Hamelaers van de vakgroep Biofysica; beste Arnold en Jan, hartelijk dank voor jullie introductie in de reologie. Hoewel dit onderwerp niet aan bod is gekomen in dit proefschrift, wil dat niet zeggen dat er niets meer met de data gedaan zal worden. Helaas is Jan Meinders te vroeg van ons afgenomen.

Jeroen, bedankt voor de materiële ondersteuning.

Prof. Dr. H. ten Cate en Dr. J. Govers-Riemslag van de vakgroep Biochemie; beste Hugo en Jose, ik ben blij dat Anna's project gecontinueerd is en geresulteerd heeft in een publicatie over fibrinolyse in FGH.

Dr. O. Bekers en Prof. Dr. M.P. van Dieijen-Visser van de vakgroep Klinische Chemie; beste Otto en Marja, bedankt voor o.a. de de apo E genotyperingen. Het E2/E2 genotype is fascinerend, daar moeten we het in de toekomst misschien nog maar eens over hebben. 
Prof. Dr. A.F. Stalenhoef en Dr. J. de Graaf, UMC Radboud te Nijmegen; Anton en Jacqueline, ons parabool manuscript is het eerste resultaat van onze samenwerking. Dat er nog vele gezamenlijke projecten mogen volgen!

Prof. Dr. J. Dekker en Prof. Dr. R.J. Heine, Vrije Universiteit Amsterdam; beste Jacqueline en Rob, hartelijk dank dat ik gebruik heb mogen maken van de wereldberoemde 'Hoorn-data'.

Dr. R.M. Cantor, Dr. A.J. Lusis, Department of Human Genetics, University of California Los Angeles, USA; dear Rita, I was initially afraid that it would not be feasible to accomplish our predefined goals in a 6-weeks short visit, but thanks to your help and patience I managed. Thanks for your introduction to linkage analysis. I very much appreciate your help afterwards as well. Jake, your hospitality and enthusiasm has struck me. Thank you very much for letting me attend your lab meetings and for giving me the opportunity to discuss my data with you while enjoying margaritas.

Dear Naoko and Bruce, thank you very much for showing me around in Los Angeles. It was a great experience, thanks to you!

Hoewel mijn leven de afgelopen jaren met regelmaat uit werken, werken en werken heeft bestaan, waren er gelukkig nog altijd DE Buddies, Frenkie P, Smeets en Trapetoni die voor de broodnodige afleiding hebben gezorgd. Ik hoop dat ik de komende periode wat meer tijd zal hebben om in onze vriendschap te investeren. Datzelfde geldt voor jou, Marco.

Lieve Esther, je bent het bewijs van mijn ongelijk: relaties kunnen overgaan in goede vriendschappen. Ik hoop je niet kwijt te raken.

Beste Pascal, mijn grote broer, laat de grafische vormgeving van dit proefschrift (wederom) het bewijs zijn van waar jouw kwaliteiten liggen. Heel hartelijk dank voor jouw onvoorwaardelijke hulp.

Pap en mam, er is geen moment dat jullie er niet voor me zijn geweest. Dat ben ik jullie eeuwig dankbaar. En mam: inderdaad, uiteindelijk is het allemaal goed gekomen... 


\section{Curriculum vitae}

Martijn Carolus Gertrudis Johannes Brouwers was born in Geldrop on the 8th of May 1978. In 1996, he completed his secondary education at the Augustinianum in Eindhoven and started his study Medicine at Maastricht University. During his study he did an internship 'diabetes mellitus' at the Department of Medicine at the University of Helsinki, Finland. He obtained his medical degree with honors in 2002. From October 2002 he worked for four years as a PhD student at the Laboratory of Molecular Metabolism and Endocrinology at Maastricht University of which the results are described in the present thesis. As a part of his traineeship, Martijn conducted genetic analyses at the prestigious Department of Human Genetics, University of California Los Angeles, USA, in close collaboration with Dr R. Cantor and Dr. A. Lusis. Since October 2006 he has worked as a resident at the Department of Internal Medicine at the University Hospital Maastricht and Atrium Medical Center Heerlen. 


\section{Scientific output}

\section{Original contributions}

1. Brouwers MC, Dekker JM, van Greevenbroek MM, van der Kallen CJ, Heine RJ, de Bruin TW, Stehouwer CD. Plasma triglycerides and LDL-cholesterol are related in a parabolic fashion in the general population and type 2diabetes mellitus; long-term followup results from the Hoorn study. submitted

2. Brouwers MC, Govers-Riemslag J, Schalkwijk CG, van Greevenbroek MM, van der Kallen CJ, Bekers O, van Dieijen-Visser MP, ten Oever J, Bilderbeek-Beckers MA, de Bruin TW, ten Cate H, Stehouwer CD. Plasma PAI-1 levels are independently related to fatty liver and hypertriglyceridemia in familial combined hyperlipidemia, involvement of apolipoprotein E. Thromb Res; 2007 in press

3. Brouwers MC, de Graaf J, van Greevenbroek MMJ, Georgieva AM, van der Kallen CJH, ter Avest E, Stehouwer CDA, Stalenhoef AF, de Bruin TWA. Parabolic relation between plasma triglycerides and LDL-cholesterol in familial combined hyperlipidemia; the multiple-type hyperlipidemia explained? Clin Sci (Lond); 2007 in press

4. Brouwers MC, van Greevenbroek MM, Bilderbeek-Beckers MA, Robertus-Teunissen MG,van der Kallen CJ, Stehouwer CD, de Bruin TW. Fatty liver-based identification of two distinct hypertriglyceridemic subgroups in familial combined hyperlipidemia. Metabolism. 2007 Oct; 56(10):1311-1317;

5. Brouwers MC, van Greevenbroek MM, Vermeulen VM, van Lin JM, van der Kallen CJ, de Bruin TW. Five-year follow-up of waist circumference, insulin and ALT levels in familial combined hyperlipidemia. Clin Sci (Lond). 2007 Nov; 113(9): 375-381;

6. Wang P, van Greevenbroek MM, Bouwman FG, Brouwers MC, van der Kallen CJ, Smit E, Keijer J, Mariman EC. The circulating PFEF/NAMPT/visfatin level is associated with a beneficial blood lipid profile. Pflugers Arch. 2007 Apr; 454: 971-976;

7. Brouwers MC, Bilderbeek-Beckers MA, Georgieva AM, van der Kallen CJ, van Greevenbroek MM, de Bruin TW. Fatty liver is an integral feature of familial combined hyperlipidaemia:relationship with fat distribution and plasma lipids. Clin Sci (Lond). 2007 Feb; 112(2):123-30.

8. Brouwers MC, Cantor RM, Kono N, Yoon JL, van der Kallen CJ, Bilderbeek-Beckers MA, van Greevenbroek MM, Lusis AJ, de Bruin TW. Heritability and genetic loci of fatty liver in familial combined hyperlipidemia. J Lipid Res. 2006 Dec;47(12):2799-807. 
9. Brouwers MC, Kono N, van Greevenbroek MM, van der Kallen CJ, Lusis AJ, de Bruin TW, Cantor RM. Longitudinal differences in familial combined hyperlipidemia quantitative trait loci. Arterioscler Thromb Vasc Biol. 2006 Jun;26(6):e118-9.

10. de Bruin TW, Georgieva AM, Brouwers MC, Heitink MV, van der Kallen CJ, van Greevenbroek MM. Radiological evidence of nonalcoholic fatty liver disease in familial combined hyperlipidemia. Am J Med. 2004 Jun 15;116(12):847-9.

11. Georgieva AM, van Greevenbroek MM, Krauss RM, Brouwers MC, Vermeulen VM,Robertus-Teunissen MG, van der Kallen CJ, de Bruin TW. Subclasses of low-density lipoprotein and very low-density lipoprotein in familial combined hyperlipidemia: relationship to multiple lipoprotein phenotype. Arterioscler Thromb Vasc Biol. 2004 Apr;24(4):744-9.

\section{Abstracts}

1. M. Brouwers, J. Dekker, A. Georgieva, M. van Greevenbroek, C. van der Kallen, R. Heine, T. de Bruin, C. Stehouwer. Parabolic relation between plasma triglycerides and LDL-cholesterol in the general population and patients with type 2 diabetes mellitus; 6-year follow-up results from the Hoorn study. Diabetologia, 2007; 50[Suppl1]: S9

2. M. Brouwers, M. van Greevenbroek, J. de Graaf, A. Georgieva, C. van der Kallen, E. ter Avest, C. Stehouwer, A. Stalenhoef, T. de Bruin. PO3-81 Parabolic relation between plasma triglycerides and LDL-cholesterol in familial combined hyperlipidemia: the multiple-type hyperlipidemia explained? Atherosclerosis Suppl, 2007; 8(1): 37-38

3. M. Brouwers, M.A.L. Bilderbeek-Beckers, A.M. Georgieva, C.J.H. van der Kallen, M.M.J. van Greevenbroek and T.W.A. de Bruin. W08-P-003 Fatty liver in hypertriglyceridemic subjects with familial combined hyperlipidemia: Importance of visceral fat. Atherosclerosis Suppl, 2005; 6(1): 30

4. A. Georgieva, M. van Greevenbroek, M. Brouwers, C. van der Kallen and T. de Bruin. T01-P-007 Accumulation of VLDL1 particles in plasma is the main determinant of hypertriglyceridemia. Atherosclerosis Suppl, 2005; 6(1): 135

\section{Oral presentations}

1. 43rd EASD annual meeting 2007; Parabolic relation between plasma triglycerides and LDL-cholesterol in the general population and type 2 diabetes mellitus; 6 -year follow-up results from the Hoorn study 
2. 10th Dutch Atherosclerosis Society 2007; Parabolic relation between plasma triglycerides and LDL-cholesterol in familial combined hyperlipidemia: the multiple-type hyperlipidemia explained?

3. 9th Dutch Atheroslerosis Society 2006; Quantitative trait linkage analysis for fatty liver in familial combined hyperlipidemia (awarded with DAS fellowship) 
Appendix A.

List of abbreviations 
ACC

AGPAT

ALP

ALT

AMPK

apo

AST

ATPIII

BMI

CE

CETP

ChREBP

CPT-1

CRP

CT

DGAT

FAS

FCHL

FFA

FHBL

GPAT

HAART

$\mathrm{HC}$

HDL

HE

HL

HOMA-IR

HSL

HSPG

HTG

IDL

Il-6

LEPR

LDL
acetyl-CoA carboxylase

acylglycerol-phospate acyltransferase

atherogenic lipoprotein phenotype

alanine aminotransferase

AMP-activated protein kinase

apolipoprotein

aspartate aminotransferase

Adult Treatment Panel III

body mass index

cholesteryl ester

cholesteryl ester transfer protein

carbohydrate regulatory element binding protein

carnitine palmitoyl transferase 1

C-reactive protein

computed tomography

diacylglycerol acyltransferase

fatty acid synthase

familial combined hyperlipidemia

free fatty acid

familial hypobetalipoproteinemia

glycerol-phosphate acyltransferase

highly active anti-retroviral therapy

hypercholesterolemia

high-density lipoprotein

Haseman-Elston

hepatic lipase

homeostasis model assessment insulin resistance

hormone sensitive lipase

heparan sulfate proteoglycan

hypertriglyceridemia

intermediate-density lipoprotein

interleukin 6

leptin receptor

low-density lipoprotein 
LOD $\quad \log$ of odds

LPL lipoprotein lipase

LRP LDL-related protein

LXR liver X receptor

MGAT monoacylglycerol acyltransferase

MRI

magnetic resonance imaging

MRS

magnetic resonance spectroscopy

MTP

microsomal triglyceride transfer protein

NAFLD

nonalcoholic fatty liver disease

$\mathrm{NASH}$

NL

nonalcoholic steatohepatitis

NPY

normolipidemia

NTG

neuropeptide $\mathrm{Y}$

normotriglyceridemia

OGTT

oral glucose tolerance test

OR

odds ratio

PAI-1

plasminogen activator inhibitor 1

PPAR

peroxisome proliferator-activated receptor

PPH-1

phosphatidate phosphohydrolase 1

PUFA

polyunsaturated fatty acid

QTL

quantitative trait locus

RXR

retinoid X receptor

SAT-US

subcutaneous adipose tissue measured with ultrasound

SCD-1

sd stearoyl-CoA desaturase 1

SD

small-dense

sICAM-1

standard deviation

SKF

soluble intercellular adhesion molecule 1

SREBP-1C

skinfold

SREBP-1C

sterol regulatory element binding protein $1 \mathrm{C}$

TG

triglycerides

TNF

tumor necrosis factor

tPA

tissue plasminogen activator

US

ultrasound

USF-1

upstream stimulatory factor 1 
VAT-US visceral adipose tissue measured with ultrasound

VLDL very low-density lipoprotein

vWF

von Willebrand factor

WHO

World Health Orginazation

WHR

waist-to-hip ratio 
Appendix B.

Linkage results for fatty liver 

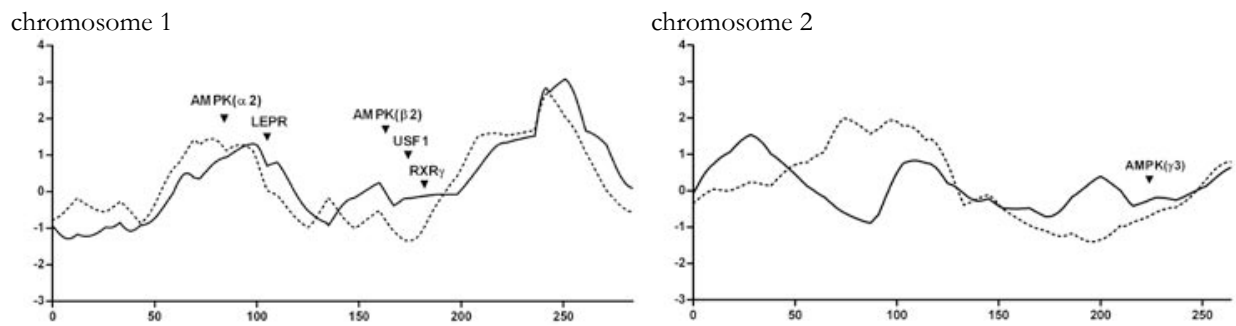

chromosome 3
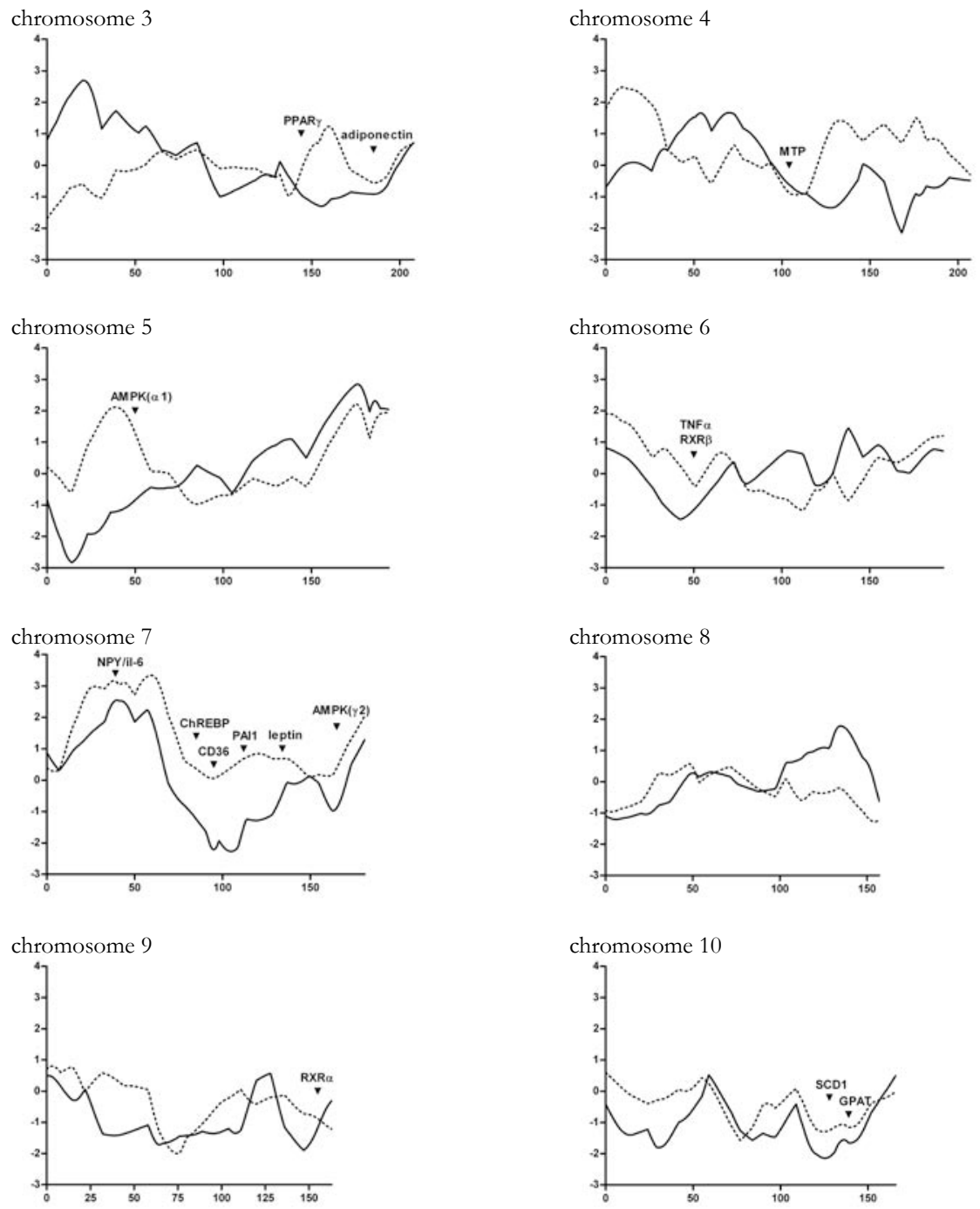
chromosome 11

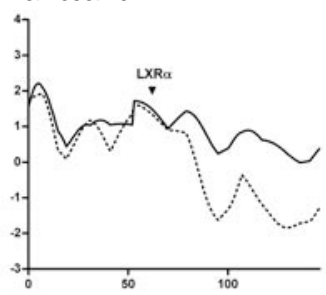

chromosome 14

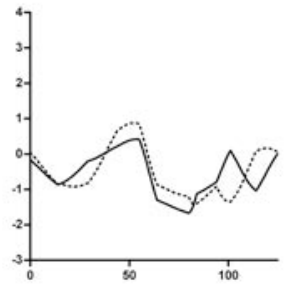

chromosome 17

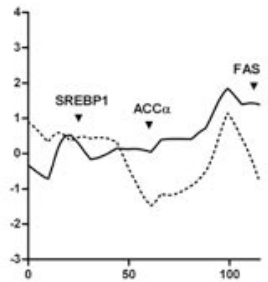

chromosome 20

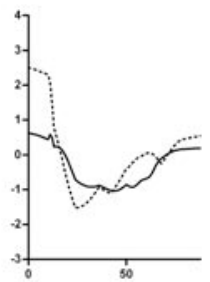

chromosome 12

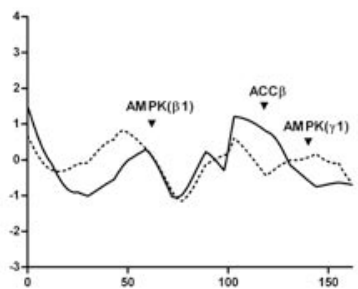

chromosome 15

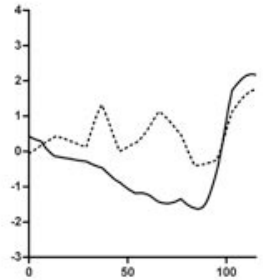

chromosome 18

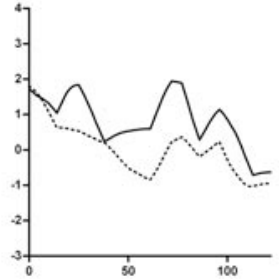

chromosome 21

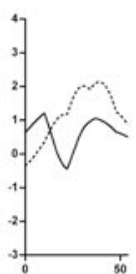

chromosome 13

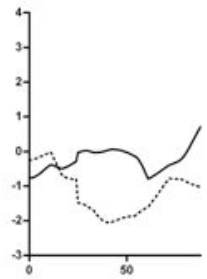

chromosome 16

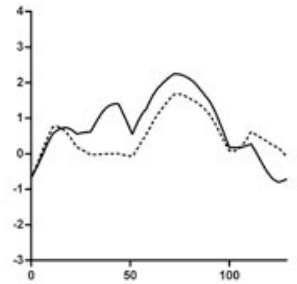

chromosome 19

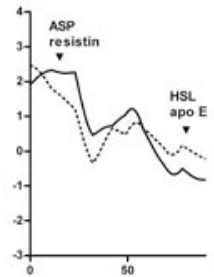

chromosome 22

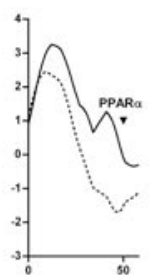

Appendix B.

Linkage results for fatty liver (ultrasound; solid lines) and log ALT (dashed lines) in the male FCHL population, as presented in paragraph 2.1.3, in relation to transcription factors, enzymes and hormones that are likely to be involved in the development of fatty liver 
